\title{
Research Commons
}

http://researchcommons.waikato.ac.nz/

\section{Research Commons at the University of Waikato}

\section{Copyright Statement:}

The digital copy of this thesis is protected by the Copyright Act 1994 (New Zealand).

The thesis may be consulted by you, provided you comply with the provisions of the Act and the following conditions of use:

- Any use you make of these documents or images must be for research or private study purposes only, and you may not make them available to any other person.

- Authors control the copyright of their thesis. You will recognise the author's right to be identified as the author of the thesis, and due acknowledgement will be made to the author where appropriate.

- You will obtain the author's permission before publishing any material from the thesis. 
The use of upper-body intermittent, sequential pneumatic compression arm-sleeves on recovery from exercise in wheelchair athletes

\author{
A thesis \\ submitted in partial fulfilment \\ of the requirement for the degree \\ of \\ Master of Health, Sport \& Human Performance \\ at \\ The University of Waikato \\ by
}

Alison Oliver

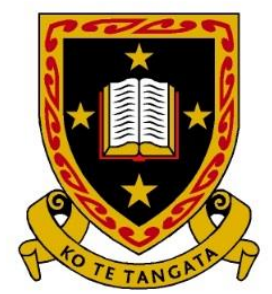

THE UNIVERSITY OF

WAIKATO

Te Whare Wänanga o Waikato

2020 


\begin{abstract}
Wheelchair basketball and wheelchair rugby are two of the most popular sports for athletes with a disability. Despite this, recovery-based research is sparse in the disability sport sector, as most studies have concentrated on the recovery needs of able-bodied athletes. Existing research has investigated several recovery methods, which the first part of this thesis discusses in a literature review. One of the recovery methods included in this review is a relatively new recovery tool known as intermittent sequential pneumatic compression (ISPC). Initially developed for use in medical settings, ISPC is believed to increase blood flow, therefore limiting inflammation/swelling and enhancing the removal of metabolic by-products associated with exercise. The second part of this thesis includes an original study investigating the influence of ISPC on recovery in wheelchair team sport athletes. Eleven wheelchair basketball and rugby players volunteered to participate in this study and were required to take part in two trials (either passive control or ISPC recovery) separated by one week. During ISPC recovery, participants wore the sleeves on both arms for 20 minutes. Grip strength, pressureto-pain threshold (PPT), medicine ball throw, wheelchair sprints, repeated sprints and blood lactate (BLa) were measured pre-exercise, post-exercise, and post-recovery. Subjective muscle soreness and fatigue were also assessed at these time points, as well as 24 hours post-exercise. The exercise session consisted of 20 minutes of high intensity drills, designed to simulate the physiological demands of wheelchair team sport. Analysis revealed ISPC to positively influence subjective measures of muscle soreness and fatigue, with negligible effects on performance recovery and physiological measures when compared to a control trial. The final section of this thesis summarises the overall findings of the thesis, as well as providing practical applications and future research.
\end{abstract}




\section{Acknowledgements}

I would like to take this opportunity to thank those who have contributed to this thesis.

To my supervisor Dr. Matthew Driller, thank you for guiding me through this challenging and often relentless enterprise. Your time and expertise have been invaluable, and greatly appreciated.

To the participants who volunteered their precious time to take part in this study, thank you. I would not have been able to do it without all your help.

To all my family, friends and colleagues who have supported, encouraged and inspired me to persevere, thank you will never seem enough.

Lastly to my parents, who instilled in me the importance of education in creating opportunities, determination, drive and at times pure stubbornness to get the job done. 


\section{Thesis Organisation}

This thesis consists of three chapters. Chapter 1 (literature review) describes the development of wheelchair basketball and wheelchair rugby, as well as a brief introduction to the rules and demands of both sports. Athletic recovery is also introduced and discussed, outlining the literature relating to the efficacy of different recovery methods currently used by athletes. Chapter 2 compromises of an original study on the effects of intermittent sequential pneumatic compression on recovery in wheelchair team sport athletes. This chapter is formatted in the required style of journal it was submitted to the American Journal of Physical Medicine and Rehabilitation. Consequently, some information may be repeated throughout this thesis. Chapter 3 provides a summary of the overall findings, along with practical applications, limitations and recommendations for future research. 


\section{Contents}

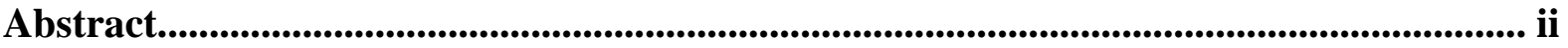

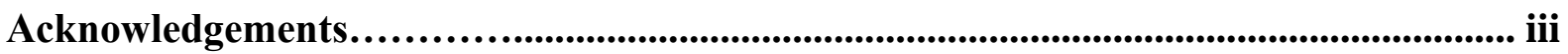

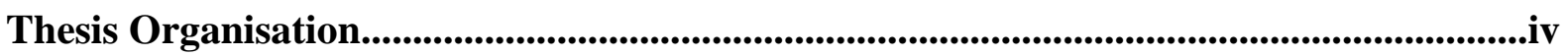

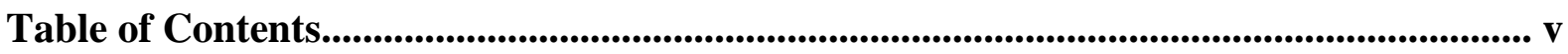

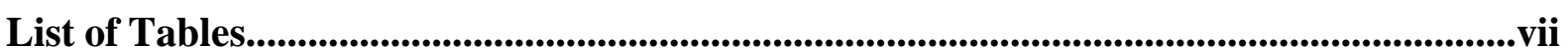

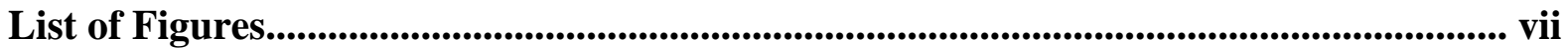

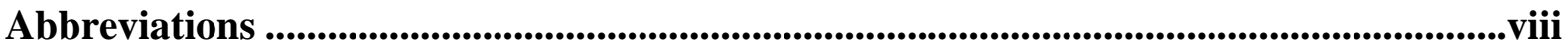

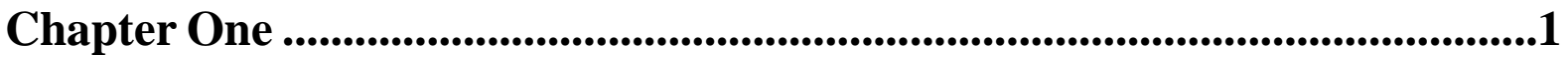

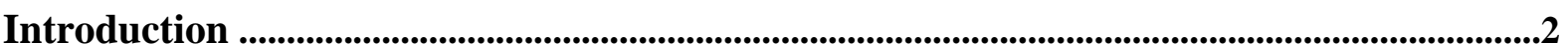

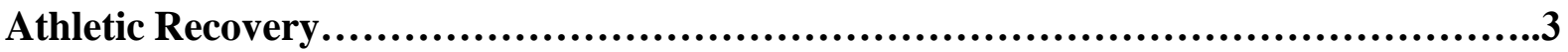

Adaptation to Training......................................................................4

Delayed Onset Muscle Soreness................................................. 5

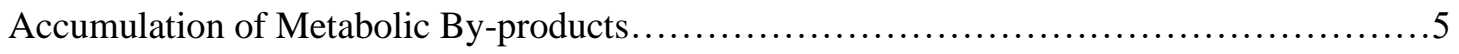

Decreased Energy Substrates....................................................

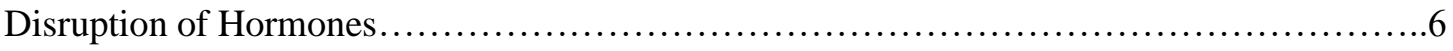

Training and Recovery Balance.....................................................6

Fatigue....................................................................................8

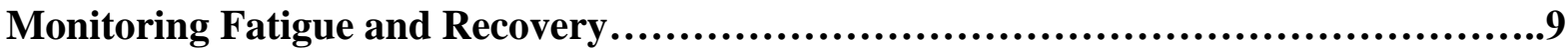

Recovery Methods..................................................................12

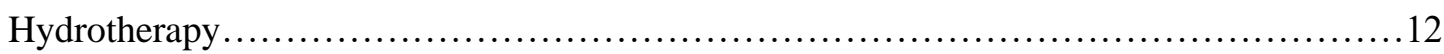

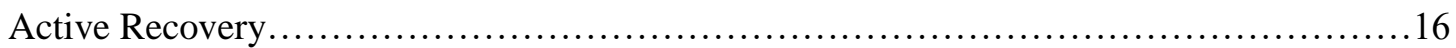

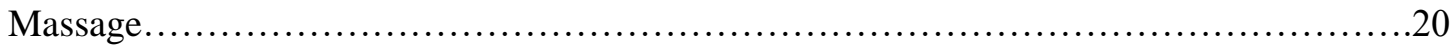

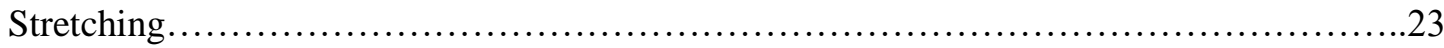

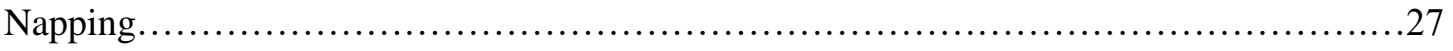

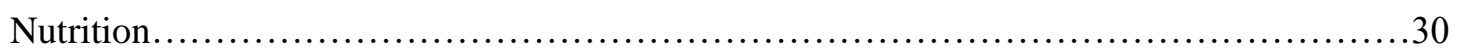

Compression Garments.............................................................

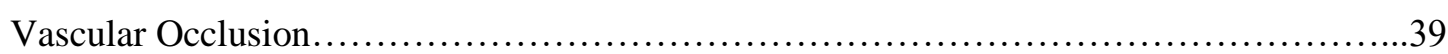

Intermittent Sequential Pneumatic Compression.......................................41

The Placebo Effect..................................................................45

Recovery in Athletes with a Disability ............................................46

Monitoring Fatigue and Recovery in Athletes with a Disability $\ldots . . . \ldots \ldots \ldots \ldots \ldots \ldots \ldots . . . . .47$

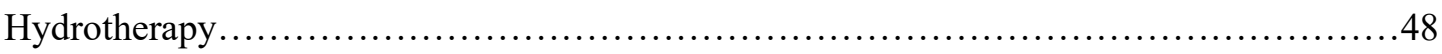

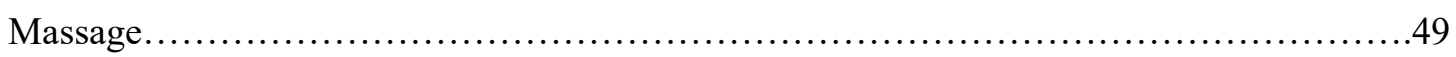




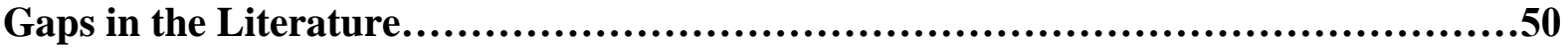

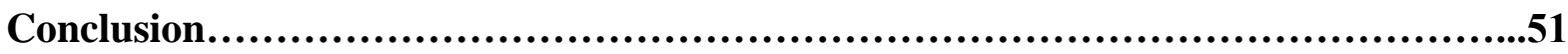

Chapter Two.................................................................................53

Abstract...................................................................................54

What is Known....................................................................54

What is New.............................................................................54

Introduction............................................................................55

Methods..........................................................................57

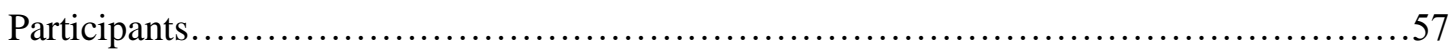

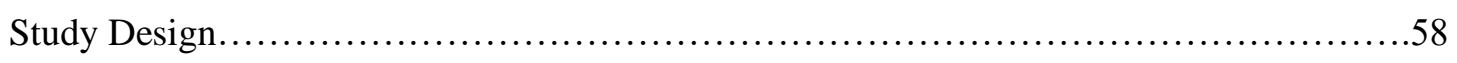

Maximal Medicine Ball Chest Throw..............................................60

Wheelchair Sprints and Repeated Sprints .............................................6

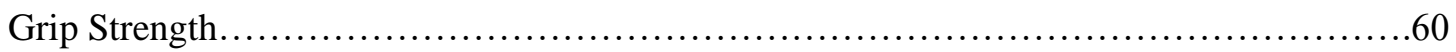

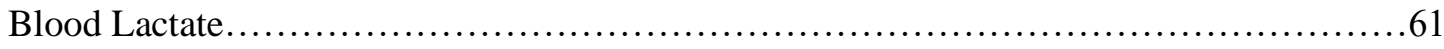

Pressure to Pain Threshold.............................................................61

Muscle Soreness and Fatigue Scales.............................................62

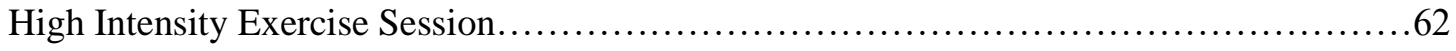

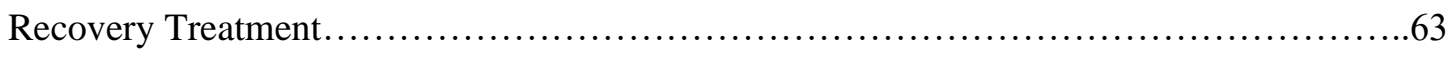

Pressure Monitoring ..............................................................6

Statistical Analysis.............................................................64

Results..........................................................................64

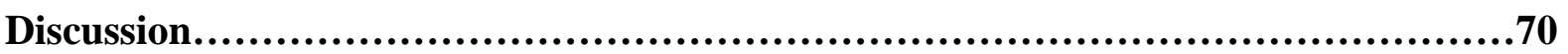

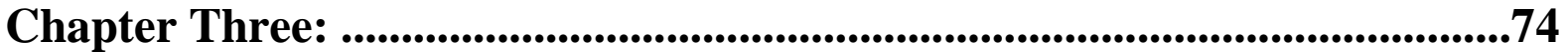

Summary............................................................................75

Practical Applications................................................................76

Limitations.............................................................................77

Future Research.......................................................................78

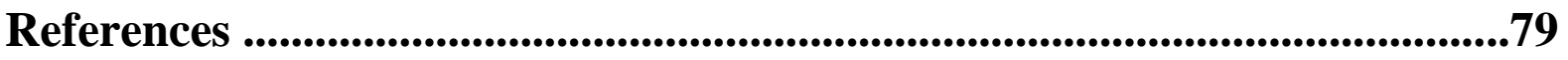

Appendices ................................................................................................................................102

Appendix 1........................................................................102

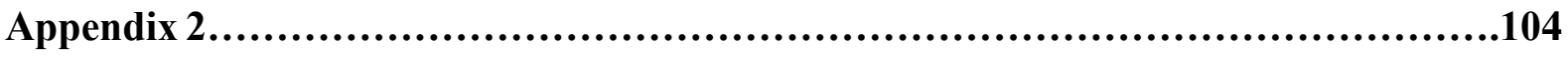




\section{List of Tables}

Table 1 - Participant characteristics and disability classifications ........................58

Table 2 - Comparison of intermittent sequential pneumatic compression (ISPC) and control $(\mathrm{CON})$ interventions at all time points. Data is presented as means \pm SD.

Table 3 - Comparison of all measures (post-recovery and 24h post-exercise) compared to postexercise (pre-recovery) values. Data presented as raw difference in values (mean $\pm 90 \%$ confidence intervals) with Cohen's $d$ effect sizes (and 90\% confidence intervals) for comparison between intermittent sequential pneumatic compression (ISPC) and control (CON) interventions.

\section{List of Figures}

Figure 1. The stress/stimulus response adapted from the General Adaptation Syndrome (GAS) model................................................................................ 8

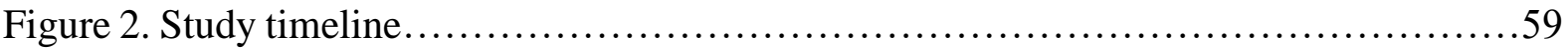

Figure 3. Landmarks for measurement of pressure-to-pain threshold using the algometer

Figure 4. Positioning during ISPC recovery

Figure 5. Comparison of intermittent sequential pneumatic compression (ISPC) and control (CON) interventions across time points for a) perceived muscle fatigue, and; b) perceived muscle soreness 

Abbreviations
ADP
Adenosine diphosphate
AOP
Arterial occlusion pressure
ATP
Adenosine triphosphate
AVA
Arteriovenous anastomoses
BLa
Blood lactate
BP
Blood pressure
CK
Creatine kinase
CMJ
Countermovement jump
CON
Control
$\mathrm{CP}$
Cerebral palsy
CRP
C-reactive protein
CWI
Cold water immersion
CWT
Contrast water therapy
DOMS
Delayed onset muscle soreness
DVT
Deep vein thrombosis
EIMD
Exercise induced muscle damage
EMG
Electromyography
EXP
Experimental
FFA
Free fatty acids
GAS
General Adaptation Syndrome
HR
Heart rate
HRR
Heart rate recovery
HRV
Heart rate variability
IL-6
Interleukin 6 
ISPC

LT

MD

NFO

NIRS

OTS

$\mathrm{PCr}$

$\mathrm{pH}$

PNF

PPT

RESTQ-sport

RFD

RHR

ROM

RPE

RPM

SCI

SRPE

SSC

SWS

TTE

$\mathrm{VO}_{2} \max$

$\mathrm{VO}_{2}$ peak

$1 \mathrm{RM}$
Intermittent sequential pneumatic compression

Lactate threshold

Muscular dystrophy

Non-functional overreaching

Non-invasive near-infrared spectroscopy

Overtraining Syndrome

Phosphocreatine

Power of hydrogen

Proprioceptive neuromuscular facilitation

Pressure to pain threshold

Recovery Stress Questionnaire for Athletes

Rate of force development

Resting heart rate

Range of motion

Rate of perceived exertion

Rotations per minute

Spinal cord injury

Session rate of perceived exertion

Stretch-shortening cycle

Slow wave sleep

Time to exhaustion

Maximal oxygen uptake

Peak oxygen uptake

One repetition maximum 
CHAPTER ONE - Literature Review 


\section{Introduction}

Wheelchair basketball was first played at military hospitals in California and Massachusetts, United States in 1945 following World War II, to assist injured soldiers during rehabilitation (International Wheelchair Basketball Federation, 2016). At the same time a similar game was developed by Dr Ludwig Guttmann at the Stoke Mandeville hospital, a spinal rehabilitation centre, in Great Britain (International Paralympic Committee, 2016). Over a 10-year period the game continued to grow in popularity, with the National Wheelchair Basketball Association being formed in 1949 in the United States. The inaugural United States national championships took place in Illinois that same year, starting out with just six teams (International Wheelchair Basketball Federation, 2019). In 1955 the first International Stoke Mandeville Games took place, marking the beginning of international competition and initiating the growth of the sport throughout Europe. The sport was included in the first Paralympic Games in 1960 (International Wheelchair Basketball Federation, 2019). Wheelchair basketball is now played in approximately 100 countries around the world, with top international fixtures often televised (International Paralympic Committee, 2016).

In comparison, wheelchair rugby was not developed until 1977 in Winnipeg, Canada. Initially it was primarily for quadriplegic athletes with spinal cord injuries (SCI), who had limited upper body function (International Wheelchair Rugby Federation, 2019), however athletes with other disabilities such as cerebral palsy (CP), muscular dystrophy (MD), polio and multiple amputations regularly participate (Goosey-Tolfrey \& Leicht, 2012). The first Canadian national championships took place in 1979, along with the game making its first appearance in the United States. The first international event was held in 1982, involving teams from Canada and the United States and by 1989, Great Britain had formed a team (International Paralympic Committee, 2016). Similarly to wheelchair basketball, its popularity grew quickly and in 1990 wheelchair rugby made its debut at the Stoke Mandeville Games. In 1993 the International Wheelchair Rugby Federation became an official governing body for the sport. A year later it was officially classified as a Paralympic sport, however it was not part of the medal programme until the Paralympic Games in 2000. At present, wheelchair rugby is played in over 40 countries (International Wheelchair Rugby Federation, 2019).

Like many other team sports, wheelchair basketball and rugby are games which demand a multitude of physical (speed, strength, power, endurance), technical (passing, throwing, 
catching), mental/psychological and tactical skills (Malone et al., 2011). Both sports involve multiple high intensity efforts consisting of stopping, starting, changes in direction and sprinting. The ability to repeat these efforts frequently throughout a game with little rest requires both anaerobic and aerobic fitness (Ozmen, Yuktasir, Un Yildirim, Yalcin \& Willems, 2014). Wheelchair basketball games consist of four 10-minute quarters and generally adhere to the same rules as able-bodied basketball (International Wheelchair Basketball Federation, 2019). Wheelchair rugby games consist of four 8 -minute quarters and require players to pass over the goal line while in possession of the ball. Like able-bodied rugby, wheelchair rugby is a full contact sport with collisions considered a fundamental part (Malone, MorgulecAdamowicz \& Orr, 2011).

An important feature in both wheelchair basketball and rugby (as well as many other disability sports) is the classification system. The aim of this system is to ensure fairness and provides all players the opportunity to play without being limited by their functional ability (International Wheelchair Rugby Federation, 2015). In wheelchair basketball, each player is assigned a number between 1.0 and 4.5 (1.0 equates to the lowest functional ability). These numbers are based on each individual player's level of impairment combined with their trunk, lower limb and upper limb function and how they influence each player's ability to perform the skills required for the game. These skills include passing, dribbling, catching and shooting the ball, as well as pushing themselves around the court in their wheelchair. During a game each team is allowed five players on the court, however the combined classification scores cannot be greater than 14.0 (International Wheelchair Basketball Federation, 2014). The wheelchair rugby classification system is almost identical to the wheelchair basketball system, with some differences. Generally, wheelchair rugby players have more limitations, particularly with their trunk and upper limb function so each player is assigned a number between 0.5 and 3.5. During games, each team is only allowed four players on the court, with the combined classification score not exceeding 8.0 (International Wheelchair Rugby Federation, 2015).

\section{Athletic Recovery}

Wheelchair basketball and rugby tournaments take place over the course of several days (International Wheelchair Basketball Federation, 2016; International Wheelchair Rugby Federation, 2015). Often two games are scheduled on the same day, leaving very little rest time (International Wheelchair Basketball Federation, 2016; International Wheelchair Rugby 
Federation, 2015). Furthermore, during particular training blocks, athletes competing at a high level may be required to complete multiple sessions per day. Therefore, in order to maintain a high level of performance throughout tournaments and demanding training blocks, athletes need to be able to recover efficiently (Halson, 2013). Recovery from athletic training is a complex process and dependent upon many factors such as the demands of the sport, length and intensity of the training session and whether the athlete completes multiple sessions each day (Kellmann, Bertollo, Bosquet, Brink, Coutts, Duffield, Erlacher, Halson, Hecksteden, Heidari, Kallus, Meeusen, Mujika, Robazza, Skorski, Venter \& Beckmann, 2018). The resources available to the individual will also determine the recovery method(s) used as some methods are considerably more accessible and cost effective than others.

Kellmann and Kallus (2001) describe recovery as "an inter-individual and intra-individual multi-level (e.g., psychological, physiological, social) process in time for the re-establishment of performance abilities". Not only does this reiterate the multifactorial nature of recovery, it also emphasises the importance of recovery methods that are customised to each individual athlete (Kellmann, 2010). The importance and necessity of recovery has become more apparent in recent years as the interest in this training concept has increased substantially (Montgomery, Pyne, Hopkins, Dorman, Cook \& Minahan, 2008). The subsequent research that has taken place has resulted in recovery being considered a vital part of elite and recreational level athlete training programmes (Peake, 2019; Kellmann et al., 2018). Many national sporting organisations are now dedicating valuable resources to recovery protocols. Recovery centres first started opening at the United States Olympic Training Centre in Colorado, Australian Institute of Sport in Canberra and the Queensland Academy of Sport in Brisbane (Kellmann. 2010). Many centres with similar facilities have now been opened globally. In fact, most major professional sports teams have opened their own recovery centres at their training bases.

\section{Adaptation to Training}

Following a training session or competitive event, many physiological processes within the body, such as those that maintain homeostasis within the nervous, thermoregulatory, endocrine and cardiovascular systems, are disrupted (Peake, 2019). The major consequences of intense training and competition are skeletal muscle damage, accumulation of metabolic by-products, decreased substrates/fuel and disruption of hormones (Kenney, Wilmore \& Costill, 2012). Optimal recovery methods assist the body in repairing damage and returning to homeostasis, 
meaning all physiological processes return to pre-exercise levels. Some of these processes, such as heart rate recovery take minutes, others such as lactate clearance take longer, often up to an hour depending on individual fitness levels (Luttrell \& Halliwill, 2015).

\section{Delayed Onset Muscle Soreness}

Delayed onset muscle soreness (DOMS) refers to muscle soreness that often manifests itself 24 to 48 hours after an intense training session and is thought to occur when muscles are exposed to excessive eccentric force (Dupuy, Douzi, Theurot, Bosquet \& Dugue, 2018). As well as soreness, athletes may also experience swelling, restricted range of motion (ROM) and pain. Structural damage at the cellular level and inflammatory reactions are believed to be the main causes of DOMS (Kenney et al., 2012). In terms of performance, DOMS can limit an athlete's ability to generate peak muscle forces. Glycogen resynthesis is also diminished as the muscle regeneration process takes precedence, meaning muscle fuel storage is temporarily limited (Kenney et al., 2012).

\section{Accumulation of Metabolic By-products}

The accumulation of metabolic by-products is another consequence of intense training particularly during activity which places high demand on the ATP-PCr and glycolytic systems (Kenney et al., 2012). Due to the intense nature of wheelchair basketball and wheelchair rugby both these systems are used to some extent during games. The ATP-PCr system provides an immediate source of energy for sudden intense activity such as sprinting and changes of direction. The glycolytic system can sustain the production of adenosine triphosphate (ATP) for slightly longer periods. Energy from this process can sustain intense activity, such as offensive plays or periods of defensive pressure, for 1-2 minutes. If demand is too high, the increase in acid concentration within the muscle can negatively impact glycolysis and can impair calcium binding capacity, a process needed for muscle contraction (Kenney et al., 2012). As a result, recovery methods must accommodate and enhance the processes required for the restoration of these energy systems (Kellmann et al., 2018).

\section{Decreased Energy Substrates}

Energy is predominantly provided by two of the three basic fuel sources, carbohydrates and fats (Peake, 2019). As multiple short, intense bursts of activity are a frequent feature of wheelchair basketball and wheelchair rugby, carbohydrates are the primary source of fuel as they provide an immediate source of energy required for intense activity (Kenney et al., 2012). 
However, carbohydrate stores are limited and only able to fuel approximately 60 to 90 minutes of continuous, intense exercise (Burke, 2010; Jensen, Rustad, Kolnes \& Lai, 2011). During a multi-day tournament or training blocks consisting of multiple sessions per day, carbohydrate stores can be depleted (Williams \& Rollo, 2015). This makes replenishing stores during recovery periods essential, as performance during subsequent sessions and/or games will be compromised if stores are inadequate (Peake, 2019).

\section{Disruption of Hormones}

Hormonal disruption can be a consequence of intense exercise and is typically influenced by the type of exercise and energy systems involved (Hackney \& Lane, 2015), particularly the responses of catecholamines (epinephrine and norepinephrine), testosterone and cortisol (Hackney \& Lane, 2015). Generally, catecholamines are responsible for the fight or flight response, a reaction controlled by the sympathetic nervous system. Many of the acute effects of exercise, such as increased heart rate (HR), metabolic rate and respiration are triggered by this response (Kenney et al., 2012). Likewise, another hormone released during exercise is cortisol. As the body views exercise as a type of stress, this stimulates the release of cortisol into the bloodstream (Cofre-Bolados, Requen-Lopez, Herrera-Valenzuela, Orihuela-Diaz, Garcia-Hermoso \& Hackney, 2019). In manageable amounts, cortisol is needed for many processes. Two vital roles include prompting gluconeogenesis and mobilising free fatty acids (FFA) to ensure fuel is available for exercise (Hackney \& Walz, 2013). However cortisol is a catabolic hormone, meaning when excessive concentrations are present, lean muscle may be broken down and overall maladaptation to training can be compromised (Hackney \& Walz, 2013). High loads of endurance training above 60 percent of maximal aerobic power, and anaerobic exercise above maximum oxygen intake have been linked with high cortisol concentrations (Paccotti, Minetto, Terzolo, Ventura, Ganzit, Borrione, Termine \& Angeli, 2005). Lowering cortisol following exercise could reduce any negative catabolic effects, which may impair recovery (Hackney \& Walz, 2013).

\section{Training and Recovery Balance}

Ensuring their bodies have recovered sufficiently in between sessions allows athletes to train at the optimal intensity to encourage adaptations and enhance the desired performance benefits (Dupuy et al., 2018). The inclusion of adequate recovery sessions in an athlete's training programme has also been found to reduce the risk of injury (Kellman, 2010). Finding the 
appropriate balance between training load and recovery is difficult. A position statement by the European College of Sport Science describes three stages athletes may experience if an imbalance occurs. The first stage, functional overreaching (FO) refers to the short-term decrease in performance following a bout of training, which can be reversed with rest and recovery (Meeusen, Duclos, Gleeson, Rietjens, Steinacker \& Urhausen, 2006). It is during this rest period that the body responds to the training stress and adapts to the load it was placed under, ideally increasing capacity in anticipation for the next session. This is known as the supercompensation effect (Cunanan, DeWeese, Wagle, Carroll, Sausaman, Hornsby, Haff, Triplett, Pierce \& Stone, 2018). If subsequent training sessions were to take place before the supercompensation effect, specifically, during the recovery phase, overtraining may occur (Cunanan et al., 2018). This phase of supercompensation has been described as part of a model proposed by Hans Selye (Figure 1) known as the general adaptation syndrome (GAS) model (Cunanan et al., 2018). The GAS is based upon the relationship between stress and adaptation, a concept that was able to be applied to all living beings, including in the athletic training setting (Cunanan et al., 2018). A key idea of the GAS model is that for adaptations (neuromuscular, musculoskeletal and metabolic) to occur, the athlete must be unaccustomed to the stress or training stimulus being applied. This results in the shock phase, which causes acute fatigue in response to the training stimulus (McGuigan, 2017). The resistance phase follows, where the body returns to homeostasis as all physiological processes have been restored to pretraining levels. It is during this phase that the adaptations occur. This represents the body's way of protecting itself from future disruption of its homeostatic condition. As long as sufficient recovery takes place, the supercompensation phase (as described as above) follows (McGuigan, 2017). A further key idea of the GAS model is that it does not follow a linear pattern. Individual athletes can shift between phases over various time periods (McGuigan, 2017). A related model, known as the scissors recovery model, developed by Professor Michael Kellmann, describes the link between stress-states and recovery. The main principle of this model refers to the interdependent relationship between stress and recovery. Ideally, as the training stimulus (stress) increases, recovery must increase simultaneously, otherwise stress will become chronic and training adaptations will be limited or non-existent (Kellmann, 2002). A secondary feature of this model describes circumstances similar to the previously discussed supercompensation effect. High levels of stress can induce adaptations when combined with appropriate recovery. Without proper recovery, stress will accumulate and may lead to overtraining (Kellmann, 2002). If intense training were to continue without adequate rest, the second stage known as non-functional overreaching (NFO) may occur and could cause performance decrements that 
can last weeks (Meeusen et al., 2006). At this stage symptoms such as fatigue, mood changes, hormone dysfunction and weight loss may appear (Kreher \& Schwartz, 2012). The final stage is overtraining syndrome (OTS) where performance decrements and symptoms are more severe, requiring months to recover (Meeusen et al., 2006). Multiple theories have been developed in an attempt to explain the cause of overtraining syndrome, such as the central fatigue hypothesis, oxidative stress hypothesis and the cytokines hypothesis (Kreher \& Schwartz, 2012), having said that, this is beyond the scope of this review.

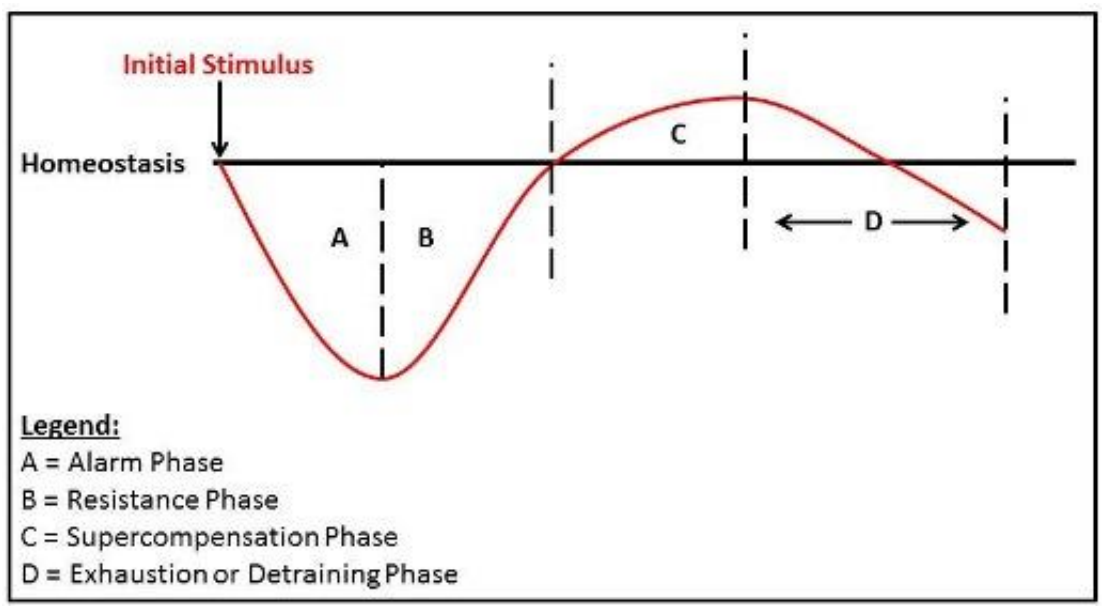

Figure 1. The stress/stimulus response adapted from the General Adaptation Syndrome (GAS) model (Contreras, 2012).

\section{Fatigue}

Fatigue is a concept frequently associated with high level athletic training and a major consequence of intense training and competition schedules (Meeusen et al., 2006). Fatigue can manifest itself through tiredness and performance decrements (Carroll, Taylor \& Gandevia, 2017). When managed correctly through recovery methods, these symptoms are temporary (Meeusen et al., 2006). Generally, fatigue presents itself as a reduction in the ability of the central nervous system to activate muscles and diminished muscle function (Carroll et al., 2017). Central fatigue describes the reduced function of the brain and spinal cord, the main components of the central nervous system (McGuigan, 2017). Conversely, peripheral fatigue refers to the reduced contractility of specific muscles (McGuigan, 2017). Central fatigue has been the topic of a considerable amount of research, which has contributed to the development of the central governor model (St Clair Gibson \& Noakes, 2004). This model proposes that the central nervous system controls the amount of force produced by the working muscles in order to maintain homeostasis, irrespective of the exercise intensity and duration, and biological 
condition of the athlete (Noakes, 2007). Additionally, perceptions of fatigue are produced by the brain with the hope that the discomfort associated with hard exercise will prevent exercise from continuing and unbalancing the homeostatic state (Noakes, 2007). The exact mechanisms of this model are yet to be agreed upon by researchers (McGuigan, 2017). Despite this, a concept that is agreed upon is that fatigue is task dependent, meaning the mechanisms that cause fatigue vary depending on the demands of the exercise being performed (McGuigan, 2017).

\section{Monitoring Fatigue and Recovery}

Common methods for monitoring fatigue can involve subjective measures such as psychological questionnaires which assess stress, mood and motivation (Robson-Ansley, Gleeson \& Ansley, 2009). Physiological and biochemical measures can provide an indication of the physical stress an athlete's body is under. These include measures such as resting heart rate (RHR), heart rate recovery (HRR), submaximal HR, creatine kinase (CK), C-reactive protein (CRP), cortisol and blood lactate (BLa) (Halson, 2014). Performance measures are also useful tools for monitoring training load. These can be sport specific by combining a multitude of physical tests which match the demands of the sport. For example, strength, speed, agility, jump, aerobic and flexibility can all be tested to indicated levels of performance recovery (Bourdon, Cardinale, Murray, Gastin, Kellmann, Varley, Gabbett, Coutts, Burgess, Gregson \& Cable, 2017). An aspect that needs to be taken into consideration when reviewing all studies is the difficulty in accurately measuring athlete recovery and fatigue, with the most common within the literature being BLa, CK, cortisol, CRP and sport specific performance measures (Halson, 2014).

BLa is a by-product of anaerobic metabolism (Kenney et al., 2012) and because it is reactive to changes in training intensity and duration, is often used to monitor the speed at which an athlete recovers. Efficient recovery typically correlates with quicker lactate clearance (Kenney et al., 2012). An issue with BLa is the influence training status may have on it. Participants with a higher LT are able to exercise at a higher intensity due to an increased lactate clearance capacity. Recruiting participants with similar levels of fitness may reduce this effect (Menzies et al., 2010) . 
CK is an enzyme that activates the shift of phosphate found in $\mathrm{PCr}$ to adenosine diphosphate (ADP) (Koch, Pereira \& Machado, 2014). EIMD can occur when CK, along with other biochemical markers such as CRP and interleukin 6 (iL-6), seeps out of the cell into the bloodstream. CK is a common measure used in practical settings as it can indicate the severity of muscle tissue damage sustained during exercise (Brancaccio, Maffulli \& Limongelli, 2007). Past studies have provided support of CK as a measure of EIMD in team sport environments where muscle damage is evident between pre and post-match time points (Thorpe \& Sunderland, 2012; Russell, Sparkes, Northeast, Cook, Bracken \& Kilduff, 2016) and following eccentric exercise such as resistance training or downhill running (Koch et al., 2014). There are limitations with $\mathrm{CK}$ as individual variations can occur due to gender, age and ethnicity (Bessa, Oliveira, Agostini, Oliveira, Oliveira, White, Wells, Teixeira \& Espindola, 2016).

As discussed previously, hormone disruption can occur during and after exercise (Hackney \& Lane, 2015). Consequently hormones are another common substance used by researchers to assess physiological responses to various exercise and recovery protocols. Testosterone and cortisol are the most frequently used because of their apparent reliability in measuring endocrine response to exercise (Cofre-Bolados et al., 2019; McLellan, Lovell \& Gass, 2010). Due to its anabolic nature, testosterone can be an indicator of the level of protein signalling and muscle glycogen synthesis. As cortisol is a catabolic hormone, it works in opposition to testosterone by reducing protein synthesis and increasing the rate of protein degradation. The testosterone cortisol ratio is therefore considered a useful measure of athlete recovery (CofréBolados et al., 2019). An increase in this ratio would indicate the body is in a catabolic state. This could help in assessing the initial responses to exercise and how recovery progresses in the time after exercise (McLellan et al., 2010).

CRP is an inflammatory marker which signals the severity of inflammation present in the body, making it a useful secondary measure of muscle damage, in combination with CK (Hill et al., 2014). However it seems the inclusion of CRP as a measurement is dependent on the activity under investigation. For example, following a six-day high intensity sprint training programme, CRP levels remained low, despite CK levels increasing (Wiewelhove, Raeder, Meyer, Kellmann, Pfeiffer \& Ferrauti, 2015). Although, when compared as an inflammatory marker in contact and non-contact sport settings, CRP only increased when contact occurred, likely causing additional muscle damage (Singh, Guelfi, Landers, Dawson \& Bishop, 2011). This indicates muscle damage may need to be above a particular threshold to influence CRP, which 
arguably makes it an ideal measure for a contact sport like wheelchair rugby (Wiewelhove et al., 2015).

With reference to performance measures, these need to be functional and sport specific. Tests which assess peak power output and fatigue are thought to be the most widely used in practical settings (Bessa et al., 2016). The CMJ is a test that appears frequently throughout the literature and considered a useful indicator of neuromuscular fatigue (Claudino, Cronin, Mezencio, McMaster, McGuigan, Tricoli, Amadio \& Serrao, 2016). Assessment of sprint performance has also been identified as a reliable measure of fatigue and recovery. When EIMD is present, maximal force, ground reaction force, muscle joint stiffness and stretch-reflex sensitivity all decrease, which impairs jump and sprint performance (Wiewelhove et al., 2015). The studies which have assessed the reliability and validity of jump and sprint tests have managed to establish a 72-hour time frame in which performance fully recovers to baseline levels. This enabled researchers to verify the efficacy of these measures (Wiewelhove et al., 2015).

As well as physiological measures of recovery, measurement of an athlete's perceived feelings of recovery are often included in the study protocol as they are perhaps just as important as physiological recovery (Venter, 2014). In order to properly assess both perceived recovery from acute and chronic training, it has been suggested fatigue, stress, mood state, readiness to train and overall well-being should be evaluated (Saw, Main \& Gastin, 2015). As they are simple and non-invasive, perceived measures of recovery are regularly included in research protocols through subjective measures such as rate of perceived exertion (RPE) and numerous questionnaires, such as the Recovery Stress Questionnaire for Athletes (RESTQ-sport) (Halson, 2014). Furthermore, the use of the perceived recovery scale has been justified because of its apparent direct relationship with fluctuations in performance (Laurent, Green, Bishop, Sjokvist, Schumacker, Richardson \& Curtner-Smith, 2011) .

Measuring recovery can be a complex process, especially in a research setting where athletes from different sporting backgrounds may be involved. Therefore it is important that no single method is used as it will limit the understanding of individual recovery needs of athletes (Halson, 2014). Ideally, a combination of all these methods should be employed to identify changes in performance and could determine whether an athlete is overtraining (Halson, 2014; Kellmann et al., 2018). 


\section{Recovery Methods}

There are currently many common techniques used by athletes to accelerate recovery (Halson, 2015). Their use is dependent upon many factors such as the type of training (intensity, volume, goal of the session), demands of the sport (endurance or strength based), the resources available to the athlete and the time in between training sessions or competitive events (Halson, 2015). There have been studies conducted to gain an insight into the popularity of various techniques used by athletes (Crowther, Sealey, Crowe, Edwards \& Halson, 2017). A review by Taveres, Smith and Driller (2017) identified cold water therapies, also known as hydrotherapy, (such as ice baths and cryotherapy) as the most commonly used in rugby. A similar finding was evident in another review by Nedelec, McCall, Carling, Legall, Berthoin and Dupont (2013) who found cold water immersion (CWI) was used often by French professional football (soccer) players, particularly during periods of frequent match play. Additional techniques such as massage, active recovery and compression garments were also used (Nedelec et al., 2013). A mixed methods study by Crowther et al (2017) investigated the use of recovery techniques by Australian team sport athletes from a variety of competition levels, ranging from recreational to elite. All international level athletes, along with 63 percent of national level athletes used massage for recovery. Stretching was the most frequently used by recreational and national level athletes. In terms of effectiveness, stretching was deemed first and second respectively by these two groups (Crowther et al., 2017). This would seem logical, considering the simplicity in implementing a stretching programme. Recreational level athletes are less likely to have access to the same resources available to elite athletes (such as regular massage therapy) (Crowther et al., 2017). Pollock, Gaoua, Johnston, Cooke, Girard and Mileva (2019) analysed the training regimes of 18 elite swimmers. In terms of recovery, post-training nutrition, with a particular emphasis on carbohydrate intake, was the most prevalent. Stretching, massage, active recovery and CWI were also used in combination with post-training nutrition. Finally, a survey by Murray, Fullagar, Turner and Sproule (2018) revealed American collegiate athletes considered sleep and taking naps as the most effective. Some of these techniques, as well as others mentioned in the literature are examined below.

\section{Hydrotherapy}

Hydrotherapy encompasses a range of techniques. The most common being CWI where athletes use ice baths, and contrast water therapy (CWT), which involves alternating between hot and cold water (Ihsan, Watson \& Abbiss, 2016). CWI is thought to improve recovery by 
reducing body temperature, which may subsequently reduce central nervous system fatigue (Ihsan et al., 2016). Reducing body temperature may also benefit the cardiovascular system. The cold water is likely to cause vasoconstriction (narrowing of the blood vessels) which limits blood flow to the extremities, increases central blood volume and reduces HR (Ihsan et al., 2016). Meanwhile, CWT supposedly enhances the removal of metabolic by-products (eg. BLa). Alternating between hot and cold water causes the subsequent vasodilation and vasoconstriction to act like a pump and increase blood flow, thereby removing waste products (Pournot, Bieuzen, Duffield, Lepretre, Cozzolino \& Hausswirth, 2011). Ingram, Dawson, Goodman, Wallman and Beilby (2009) found CWI to significantly improve muscle soreness during the 24-hour post-exercise period and enabled quicker return to baseline sprint times in team sport athletes. Likewise, a similar study found CWI performed immediately after a football game also lowered muscle soreness, along with lower levels of CK, myoglobin and CRP (Ascensão, Leite, Rebelo, Magalhäes \& Magalhäes, 2011).

However there has also been some criticism of CWI and CWT as they have been classified as counter-productive. It has been argued that the inflammatory responses associated with intense exercise are necessary for the body to repair and adapt to the training stimulus, so limiting inflammation may inhibit long term/chronic adaptations to training (Yamane, Teruya, Nakano, Ogai, Ohnishi \& Kosaka, 2006; Roberts, Raastad, Markworth, Figueiredo, Egner, Shield, Cameron-Smith, Coombes \& Peake, 2015). In a strength training setting Roberts et al (2015) found CWI may cause long term reductions in strength by preventing satellite cell activity, which normally occurs after resistance training. It has been shown that an increase in satellite cell (which maintain and regenerate muscle cells) concentration corresponds with long term muscle gain (Bellamy, Joanisse, Grubb, Mitchell, McKay, Phillips, Baker \& Parise, 2014). In their study, the non-CWI group were the only group to exhibit increases in $\mathrm{Pax} 7^{+}$satellite cells after resistance training (Roberts et al., 2015). This is a chronic adaptation, rather than an acute response.

A review by Halson (2011) explored how the time available in between training sessions may affect the efficacy of hydrotherapy techniques. Included in this review were studies looking at recovery times of less than 60 minutes and up to 24 hours. One of these was completed by Schniepp, Campbell, Powell and Pincivero (2002) who investigated how CWI influenced power output, time to peak power and HR in elite cyclists. Participants performed two 30- 
second maximal sprints separated by 15 minutes of CWI or passive rest. No difference was found in time to peak power, yet there were notable differences found in the other measures. As expected, maximal HR declined at a significantly quicker rate following the CWI protocol, compared to passive recovery. However, the major finding from this study was the effect CWI had on power output. Following the CWI protocol both average and maximum power output declined, leading the investigators to speculate that CWI may be detrimental to performance (Schniepp et al., 2002). Another study reviewed by Halson (2011), conducted by Crowe, O'Connor and Rudd (2007) followed a very similar method to Schniepp et al (2002) and produced almost identical results. CWI was found to have a detrimental effect on anaerobic performance as peak power and total work were significantly lower after CWI. The limited time (15 minutes) between exercise bouts may have caused this decrease in performance. A potential mechanism for this could be the vasoconstriction and ensuing decrease in muscle blood flow caused by the cold water. If the time between exercise bouts does not allow for adequate warm up, CWI may not be an appropriate option when time is restricted (Crowe et al., 2007). However, it does seem that CWI can be beneficial after training in the heat even if the time between bouts is less than 60 minutes (Halson, 2011).

Vaile, Halson, Gill and Dawson (2008) and Peiffer, Abbiss, Nosaka, Peake and Laursen (2009) both recruited groups of elite male cyclists to investigate the influence of CWI on thermoregulation and repeated performance after longer bouts of exercise, rather than sprinting. Vaile et al (2008) required the participants to perform five different trials over the course of five weeks. Each trial consisted of the same exercise protocol. The pre-recovery session consisted of 15 minutes at 75 percent peak power, while the post-recovery session consisted of 15 minutes at 75 percent peak power and a 15-minute time trial. Both sessions took place in a temperature-controlled room set to $34{ }^{\circ} \mathrm{C}$. Each week the participants followed a different recovery routine. Three of the routines involved one minute of CWI at various temperatures $\left(10,15,20^{\circ} \mathrm{C}\right)$ followed by two minutes outside the water. This was repeated five times to make a total of 15 minutes. The fourth routine was 15 minutes of continuous CWI at $20{ }^{\circ} \mathrm{C}$ and the fifth was 15 minutes of active recovery cycling at 40 percent of peak oxygen uptake $\left(\mathrm{VO}_{2}\right.$ peak). As discussed above a primary finding was the positive effect CWI had on thermal strain, which the researchers believe enabled participants to maintain their performance during the second exercise session. Meanwhile, rather than focusing on water temperatures like Vaile et al (2008), Peiffer et al (2009) concentrated on various durations of CWI. Maximal isometric and isokinetic torque were used as measures of performance after participants spent 5, 10 and 20 
minutes submerged in $14{ }^{\circ} \mathrm{C}$ water. Like Vaile et al (2008) body temperature dropped after CWI, however isometric and isokinetic torque levels decreased, indicating CWI may not relieve neuromuscular fatigue and could reduce muscle function. Just like Crowe et al (2007) and Schniepp et al (2002), this could explain impaired measures of peak power and sprint ability following CWI. As CWI seems to aid athletes during endurance events, measuring performance using a longer bout of exercise may have produced different results (Peiffer et al., 2009).

When longer recovery times were investigated (between 60 minute and 24 hours), contrasting results were produced. Versey, Halson and Dawson (2011) tested three CWT protocols on subsequent cycling time trial and sprint performance, this time with two hours between cycling trials. During this period, participants completed either CWT for six minutes, CWT for 12 minutes, CWT for 18 minutes or passive rest. The most effective methods were the CWT for six and 12 minutes as time-trial total work, sprint total work and peak power all improved. Eighteen minutes of CWT produced mixed results as sprint total work increased yet time-trial total work decreased. The same group of researchers completed a similar study a year later, this time recruiting trained runners (Versey, Halson \& Dawson, 2012). The CWT protocols followed were identical, yet this time six minutes of CWT proved to be the most effective in subsequent running time-trial performance. A key difference which may explain this was the setting of the later study, which was performed outdoors in a colder temperature, compared to the indoor environment used for the cycling protocol $\left(15^{\circ} \mathrm{C}\right.$ versus $\left.23{ }^{\circ} \mathrm{C}\right)$. The colder temperature combined with the longer duration of exposure to cold water may have inhibited the possible benefits. Similarly to the studies discussed earlier, with shorter recovery periods, the colder temperatures may have prevented the participant's body temperatures from rising, which may have influenced their ability to warm up (Versey et al., 2012). In a team sport environment, Hamlin (2007) found CWT significantly decreased BLa but had no effect on repeated sprint performance in rugby players. The different energy systems required for repeated sprinting, compared to time-trial running and cycling could be a contributing factor. A more likely explanation may be the different CWT protocol used by Hamlin (2007). Versey et al (2011) and Versey et al (2012) employed full body submersion with hot and cold water, while Hamlin (2007) immersed participants to hip level in cold water and used a hot shower. Full body immersion is likely to be more effective as it allows a larger amount of skin contact with the water. This combined with hydrostatic pressure may alleviate swelling (Hamlin, 2007; Versey et al., 2012). 
When reviewing the above studies on hydrotherapy techniques, the research design should be considered, as this may be a contributing factor to inconsistent results (Halson, 2011). Factors such as the variation in water temperatures, time spent submerged in water and the use of full body and partial submersion are likely to influence results. The type of exercise being performed should also be considered, as it is becoming more accepted that cold water has detrimental effects on power and sprint-based exercise. As a result, CWI immersion in particular may not be the most appropriate acute recovery method for sports requiring power and speed. Theoretically, this would include wheelchair team sports (Halson, 2011).

\section{Active Recovery}

Active recovery is a cool down period that occurs after a training session or event and is popular among team sport athletes (Halson, 2015). It consists of low intensity aerobic activity designed to increase blood flow and accelerate the removal of BLa from the muscles. When working at a lower intensity more oxygen is available, which enables the oxidisation of lactic acid by the skeletal muscles (Menzies, Menzies, McIntyre, Paterson, Wilson \& Kemi, 2010). Walking, jogging and cycling are common choices for active recovery (Halson, 2015). Many studies have supported the use of active recovery for BLa removal following exercise, particularly in comparison to passive recovery. Franchini, Takito, Nakamura, Matsushigue and Kiss (2004) conducted a study involving elite judo athletes, and how active and passive recovery compare after anaerobic intermittent activity. Body composition and $\mathrm{VO}_{2}$ peak were calculated, but $\mathrm{BLa}$ was the primary measure. After the anaerobic exercise session BLa was found to be significantly lower following active recovery. The researchers speculated this to be caused by increases in lactate oxidation, stimulated by the active muscles (Franchini et al., 2004). A similar study by Neric, Beam, Brown and Wiersma (2009) also involved anaerobic activity but recruited a group of competitive swimmers. Following a 200-metre maximal sprint, participants either rested passively or swam at 65 percent of their sprint speed. Both protocols were 20 minutes long and four measures of BLa were taken. A baseline value pre-exercise, immediately following the sprint, 10 minutes into the recovery and at the end of the 20 -minute recovery. BLa was found to be considerably lower at all post-exercise time points during active recovery, with the 20-minute post-recovery measure being very similar to the baseline measure. As well as BLa, active recovery has also been cited as beneficial in CK clearance, particularly in high contact sports such as boxing, rugby and wheelchair rugby, where muscle damage 
frequently occurs (Gill, Beaven \& Cook, 2006). Gill et al (2006) found seven minutes of postmatch active recovery on a cycle ergometer significantly reduced CK levels compared to passive recovery. Contrasting results were produced by Suzuki, Umeda, Nakaji, Shimoyama, Mashiko and Sugawara (2004) who found no difference in CK levels between active and passive recovery. Research methodology may be a contributing factor as both studies followed different processes. The participants involved in Suzuki et al (2004) completed one hour of pool-based active recovery, as opposed to seven minutes. Additionally, rather than completing the recovery routines immediately post-match, Suzuki et al (2004) performed their routines the morning after their match, which suggests active recovery may be more appropriate as an acute recovery method.

In a slightly different study Mika, Oleksy, Kielnar, Wodka-Natkaniec, Twardowska, Kaminski and Malek (2016) explored the theory that active recovery may be more effective in reducing fatigue when opposing muscle groups are used, rather than those used during the activity. The researchers hypothesised that recruiting opposing muscle groups would still allow for optimal blood flow for BLa clearance but would enable fatigued muscles recovery. Recruited participants were either canoeists or football players, both classified as either upper or lower body dominant. Three 20-minute recovery routines were assessed, a lower body (cycle ergometer), upper body (arm ergometer) and passive recovery following a treadmill running protocol. Bioelectrical activity via electromyography (EMG), torque and power were the primary measurements. The results indicated that, following fatiguing exercise, using the same muscles during active recovery is more effective in restoring pre-exercise measures and reducing fatigue (Mika et al., 2016). Similarly to Neric et al (2009) and Menzies et al (2010), the activity performed after fatiguing exercise could have facilitated the removal of metabolic substances, which perhaps reduced muscle fatigue. Likewise, lower fatigue index scores following active recovery suggested lower motor unit recruitment than during passive recovery, which may have also contributed to reduced fatigue, and encouraged recruitment of the sufficient number of motor units for subsequent exercise (Mika et al., 2016).

A factor that is not fully agreed upon in the literature is the optimal intensity for active recovery to maximise BLa clearance (Menzies et al., 2010; Devlin, Paton, Poole, Sun, Ferguson, Wilson \& Kemi, 2014). Earlier studies used maximal oxygen uptake ( $\left.\mathrm{VO}_{2} \max \right)$ to gauge intensity, concluding that intensities ranging from 25 to 60 percent of $\mathrm{VO}_{2} \max$ were adequate (Dodd, Powers, Callender \& Brooks, 1984). This was found to be flawed because of the nonlinear 
relationship between lactate threshold (LT) and workload. More recently, research has focused on the relationship between LT and active recovery intensities, which may provide a better indication of the workload that can be maintained before lactate accumulates (Baldari, Videira, Madeira, Sergio, \& Guidetti, 2005; Greenwood, Moses, Bernardino, Gaesser, \& Weltman, 2008). Menzies et al (2010) found the optimal intensity for lactate clearance during active recovery to be 80 to 100 percent of LT. An important factor to consider when interpreting these results is the relatively low maximal BLa concentrations attained by the participants. This was possibly due to the fact that participants were of moderate fitness levels, so were unable to reach higher BLa concentrations like those of the elite level (Menzies et al., 2010). Regardless, this finding was supported by Devlin et al (2014) who found intensities between 60 and 100 percent of LT proved to be more efficient at lactate clearance than intensities below that range. For other studies which did not use BLa as a measure of recovery, different results are apparent. For example, Mika et al (2016) used measures of electrical activity, power and force production, and implemented active recovery protocols at a much lower intensity on a cycle ergometer working at 60 rotations per minute (RPM) at a load of 10 watts. Decreases in these measures, along with an increase in fatigue following exercise was reported following passive recovery. Meanwhile there was little difference between measures pre and post exercise following active recovery (Mika et al., 2016). This indicates that lower intensity active recovery may be more appropriate for reducing fatigue and restoring power and force production (Mika et al., 2016). In saying that, as Mika et al (2016) did not measure BLa it is impossible to tell if any discrepancies exist.

Investigations have also been carried out to determine whether active recovery influences glycogen resynthesis as some evidence suggests it could hinder this process. Fairchild, Armstrong, Rao, Liu, Lawrence and Fournier (2003) tested this hypothesis using a cycle ergometer protocol. Participants were required to cycle steadily at 130 percent $\mathrm{VO}_{2}$ peak for two and a half minutes, followed by a 30 second sprint. Those in the control group rested passively, and the experimental group continued to pedal for 45 minutes at 40 percent $\mathrm{VO}_{2}$ peak. Similar to other studies BLa and $\mathrm{pH}$ levels recovered much quicker during active recovery compared to passive recovery. The main difference was found in glycogen resynthesis (Fairchild et al., 2003). During passive recovery glycogen levels increased substantially (Fairchild et al., 2003). Active recovery appeared to have little effect on type II muscle fibres but had a negative effect on type I fibres as glycogen breakdown occurred (Fairchild et al., 2003). Theoretically this would seem logical, as the lower intensity exercise is more likely to 
recruit type I fibres which require glucose to function (Kenney et al., 2012). This may question the findings of Menzies et al (2010) and Devlin et al (2014) as active recovery completed at higher intensities is likely to require more fuel and may compromise glycogen replenishment (Fairchild et al., 2003).

Despite its apparent positive influence on BLa clearance, the effects active recovery has on successive performance are ambiguous. Despite improvements in BLa clearance following active recovery, Franchini et al (2004) found no difference in subsequent performance. This may be related to a proposed theory that active recovery may only be beneficial when performed for shorter periods of time (Dupuy et al., 2018). After investigating a range of active and passive recovery durations after 100 metre swimming repetitions, Toubekis, Tsolaki, Smilios, Douda, Kourtesis and Tokmakidis (2008) found five minutes of active recovery resulted in the best subsequent performance as 100 metre times following this recovery period proved to be the quickest. Shorter recovery times may have a similar physiological basis as CWI, in terms of maintaining body temperature. Having brief periods of active recovery may allow body temperature to remain elevated without needing to warm up, yet still be enough time for the restoration of the ATP-CrP energy system in preparation for subsequent activity (Toubekis et al., 2008; Kenney et al., 2012). It is possible the hydrostatic effect of the water on blood flow may have contributed to the enhanced recovery (Halson, 2015), however isolating this effect, especially with the involvement of swimmers is unlikely. Nevertheless, time periods in excess of 15 minutes have been associated with no performance benefits (Franchini et al., 2004).This includes the participants involved in the Franchini et al (2004) study who performed active recovery for 15 minutes. The competitive level of the athletes and their fitness levels may play a role in the success of active recovery. Those with lower levels of conditioning, such as recreational athletes, may not be able to recover sufficiently in shorter time periods, compared to elite athletes (Franchini et al., 2004). Fitness could also influence an athlete's ability to perform active recovery at the proposed higher intensities, without compromising their recovery (Menzies et al., 2010). The duration of active recovery may also have a similar effect on CK. Gill et al (2006) reported reduced CK concentrations after seven minutes of active recovery. Meanwhile, Suzuki et al (2004) found no improvements in CK concentrations after one hour of active recovery. 


\section{Massage}

Massage can be described as "a mechanical manipulation of body tissues with rhythmical pressure and stroking for the purpose of promoting health and well-being" (Cafarelli \& Flint, 1992, p. 1). The Western (also known as Swedish) style of massage is used most frequently with the athlete population, and also during investigations of the effectiveness of massage. This style encompasses a range of techniques known as effleurage, petrissage, friction and tapotement (Weerapong, Hume \& Kolt, 2005). These techniques are thought to aid recovery both physically and psychologically. Through the mobilisation of deep tissue, muscle tightness and tone can be reduced. Muscle tightness can impair recovery by compromising oxygen and nutrient delivery to the muscles, along with metabolite removal. Therefore the increased circulation can improve lactate clearance and reduce muscle pain and swelling (Brukner \& Khan, 2012). Due to these proposed effects, massage has been cited as beneficial in providing relief for exercise induced muscle damage (EIMD) (Brukner \& Khan, 2012).

Hilbert, Sforzo and Swensen (2003) specifically looked at how massage affects neutrophil count, along with peak torque, ROM and muscle soreness in participants experiencing symptoms of DOMS. Using an isokinetic device and eccentric muscle contractions, muscle soreness was induced in the hamstring muscles. Two hours later, participants in the experimental (EXP) group had a 20-minute massage. Based on the physiological measures, the results do not support the use of massage as there was little difference in neutrophil levels, ROM and peak torque between the EXP and control (CON) groups. This result is different to earlier studies which have used similar measures. For example, Smith, Keating, Holbert, Spratt, McCammon, Smith and Israel (1994) followed a very similar protocol but obtained contrasting results as circulating neutrophil levels remained elevated in the EXP group, implying that neutrophil margination may have been reduced. Based on this it could be assumed that inflammation and muscle damage was also reduced, therefore suggesting the efficacy of massage. Unfortunately, muscle damage was not specifically measured so this conclusion is speculative. By using measures of peak torque and ROM, Hilbert et al (2003) incorporated measures of muscle damage. However it should be mentioned that these are not direct measures of muscle damage. When Hilbert et al (2003) was published, there was evidence to support the use of these measures in assessing inflammation and function of muscle (Warren, Lowe \& Armstrong, 1999; MacIntyre, Reid, Lyster \& McKenzie, 2000). More recent research has contradicted this, as Penailillo, Blazevich, Numazawa and Nosaka (2015) found rate of force 
development (RFD) to be a superior indicator of muscle damage, when compared to peak torque.

A meta-analysis by Poppendieck, Wegmann, Ferrauti, Kellmann, Pfeiffer and Meyer (2016) identified the time between massage and the post-recovery exercise as a potentially influencing factor in the efficacy of massage. Studies which incorporated shorter recovery periods (and massages) resulted in higher incidences of improved subsequent performance. For example, Mancinelli, Davis, Aboulhosn, Brady, Eisenhofer and Foutty (2006) recruited a group of collegiate basketball and volleyball players to investigate whether massage provided relief from DOMS. Measurements included vertical jump, shuttle runs and pressure to pain threshold (PPT). Participants received a 17-minute massage, then post-recovery performance tests were started within three minutes of massage completion. The results from this study support the use of massage as perceived soreness, vertical jump and PPT improved. There was no change in the shuttle run time, however times for the CON group increased. Similar findings were discovered in a more recent study by Ali Rasooli, Koushkie Jahromi, Asadmanesh and Salesi (2012) involving elite swimmers, however the main method of measurement was BLa. Massage time was also slightly shorter at 10 minutes and post-recovery activity began immediately. Compared to passive recovery, massage reduced BLa following high intensity swimming. In comparison Hilbert et al (2003), a study in which massage proved to be ineffective, scheduled the massage two hours post-exercise. Due to these results, Poppendieck et al (2016) questioned the use of massage for long term recovery, instead it seems to provide more acute benefits, particularly for subsequent performance, or as described by Poppendieck et al (2016), a "short-term pre-massage effect."

The same meta-analysis by Poppendieck et al (2016) also attempted to summarise the effect of massage on the various types of fatigue inducing activity used by researchers. This revealed massage to have a trivial effect following endurance exercise. Dawson, Dawson and Tiidus (2004) demonstrated in a real life setting by recruiting a group of recreational runners. When massage was applied following a half marathon event, swelling, soreness and strength loss were no different to the $\mathrm{CON}$ group who received no treatment. Recovery from mixed exercise appears to benefit the most from massage (Poppendieck et al., 2016). The study by Mancinelli et al (2006) provides an example of this as fatigue was induced using a combination of strength training and intense sport specific (basketball and volleyball) drills. It may be possible that massage has opposing effects on the consequences of different types of exercise. Based on their 
meta-analysis, Poppendieck et al (2006) concluded that massage may be more effective at relieving muscular fatigue associated with resistance and interval training, rather than cardiovascular fatigue associated with endurance activity.

The psychological benefits of massage have also received some attention within this field with many studies yielding positive results (Zadkhosh, Ariaee, Atri, Rashidlamir \& Saadatyar, 2015). Poppendieck et al (2016) also concluded from their meta-analysis that the supposed benefits of shorter recovery periods (and massages) could in fact be psychological and may influence perceptual measures of recovery. Even though participants experienced little physiological recovery, Dawson et al (2004) were able to establish a link between subjective feelings of recovery and massage. This was supported by another study included in their review by Ogai, Yamane, Matsumoto and Kosaka (2008) which found that only those who received massage treatment displayed improvements in perceived muscle fatigue, stiffness and subsequent performance, despite BLa levels remaining comparable between the massage and CON groups. Consequently the researchers speculated that psychological factors were involved in this result (Ogai et al., 2008; Poppendieck et al., 2016).

Anxiety, stress and depression have been the main focus of the research examining the psychological effects of massage (Weerapong et al., 2005). It has even been argued that massage provides more benefits to the psychological recovery of an athlete, compared to physiological recovery (Hemmings, Smith, Graydon \& Dyson, 2000). During a study involving a group of boxers, Hemmings et al (2000) investigated the effect of subsequent boxing performance following either passive rest or massage. BLa, glucose concentration and perceived recovery were used as measures. Boxing performance was measured through punch force production, using a boxing ergometer device. Massage was deemed ineffective in improving subsequent performance as force production decreased and BLa levels were actually higher in the massage group. Arguably the most important finding from this study was the vast improvement in perceived recovery following massage. This is further supported by Zadkhosh et al (2015) who found significant decreases in anxiety, depression and stress levels in youth wrestlers following a series of daily massages over a 10-day period. Previous research has alluded to the possible psychological benefits of massage, particularly in reducing stress and anxiety, and improving an athlete's mood (Field, Hernandez-Reif, Diego, Schanberg \& Kuhn, 2005). Concentrations of cortisol, dopamine, serotonin (Field et al., 2005) and plasma bendorphins (Bender, Nagy, Barna, Tefner, Kadas \& Geher, 2007) have been reported following 
exercise, and may influence subjective feelings of exercise stress. It has also been speculated that the psychological effects of massage could be controlled by changes in nerve signals travelling from fatigued muscles to the brain (Ogai et al., 2008).

Despite the positive research on massage, a study by Venter, Potgieter and Barnard (2010) produced conflicting results. This study followed a qualitative approach as it required participants to complete a questionnaire. Eight hundred and ninety elite athletes from various team sport backgrounds were asked questions concerning the recovery methods they currently use, and the frequency of use. Massage was not used as regularly as other methods. Many participants, particularly those from high contact sports (rugby) only used massage on rest days. This was based on the belief that massage, particularly deep tissue, following a hard training session or match could exacerbate muscle damage that has already occurred (Barnett, 2006). According to a study by Hiruma, Umimura, Naito and Katamoto (2014) CK increased when participants received massage immediately after fatiguing calf exercises. As a result of this, athletes preferred to use massage 24 to 48 hours after exercise (Venter et al., 2010).

\section{Stretching}

Stretching is one of the most well-known and frequently utilised recovery methods by athletes from a wide range of competitive backgrounds (Crowther et al., 2017). This is likely due to it being cost effective, requires no specialist equipment and simple to implement (Crowther et al., 2017). Stretching is typically described as either static or dynamic. Static stretching involves holding a particular position for a specific period of time. Static stretching is primarily used to increase flexibility. In comparison, dynamic stretching is movement based where muscles and joints move through their range of motion. The main goal of dynamic stretching is to improve mobility and prepare the body for exercise. Traditionally dynamic stretching is incorporated into the warm up because of its preparatory nature, while static stretching is used during the cool down (Page, 2012).

All skeletal muscles contain two sensory structures, muscle spindles and golgi tendon organs, which act as protective mechanisms against injury by monitoring muscle length. Muscle spindles are made up of intrafusal fibres and nerve endings. When a muscle (and muscle spindle) is stretched suddenly, action potentials are sent to the spinal cord which activates the muscle being stretched and causes it to contract. This phenomenon is known as the stretch reflex (Kenney et al., 2012). Golgi tendon organs are located near the muscle tendon and 
respond to sudden changes in tension within the muscle-tendon complex. When a muscle contracts excessively, the golgi tendon organs react by suppressing the contracting muscle and stimulating the antagonist muscle. This reflex is known as autogenic inhibition and is considered an injury prevention mechanism as it prevents the muscle from overstretching (Kenney et al., 2012).

Despite its popularity, stretching and its effects on recovery has not received the same attention in the literature compared to other recovery methods. With its main purpose being increasing ROM and flexibility, a large proportion of the research centres around injury prevention and rehabilitation (Page, 2012; Barnett, 2006). During the early years of research, post-exercise stretching was recommended because it was believed that unaccustomed activity caused muscle soreness, spasm and ischaemic pain through lack of blood flow. Stretching was considered beneficial in relieving muscle pain and spasm by improving blood flow. The theory based on muscle spasm has since been refuted (Herbert, de Noronha \& Kamper, 2011). Despite this, blood flow and muscle soreness are the main symptoms thought to be relieved by stretching. Based on preliminary evidence on the changes stretching may elicit in muscle tissue oxygenation (Kruse \& Scheuermann, 2016; McCully, 2010; Otsuki, Fujita, Ikegawa \& KunoMizumura, 2011), Kruse, Silette and Scheuermann (2016) attempted to find a link between muscle perfusion, muscle length and oxygenation. Other studies in this area have been able to identify multiple changes in myoglobin, haemoglobin and muscle blood volume concentrations at the microvasculature level following passive stretching. Kruse et al (2016) used ultrasound and non-invasive near-infrared spectroscopy (NIRS) to measure arterial blood flow and the subsequent oxygenation-deoxygenation of the plantar flexor muscles during different stretching intensities, and to substantiate the extent to which muscle perfusion is influenced during the various stretching intensities. The primary finding of this study was that while conduit arterial blood does not decrease with stretching, blood flow at the capillary level was briefly interrupted. When the stretch was released this caused a hyperaemic response as blood flow increased at a rate determined by the intensity of the stretch (Kruse et al., 2016). It is possible this sudden influx of oxygenated blood can assist with nutrient delivery and metabolite removal (Kruse et al., 2016).

Likely to be the most apparent objective of stretching with regards to recovery, is to reduce muscle soreness and the accompanying stiffness. A study by Chen, Nosaka, Chen, Lin, Tseng and Cheng (2011) compared static stretching and proprioceptive neuromuscular facilitation 
(PNF) with a control group following muscle damaging exercise. This study took place over an eight-week period, with participants in the experimental groups performing a stretching routine triweekly. Both static stretching and PNF groups exhibited improved muscle strength recovery and ROM, decreases in muscle soreness and CK. Not only did the researchers conclude that static stretching and PNF are successful in reducing exercise-induced muscle soreness, but also that flexibility lowers vulnerability to muscle soreness. In contrast, other research does not support the use of post-exercise stretching to reduce DOMS. Robey, Dawson, Goodman and Beilby (2009) found static stretching to have no effect on muscle soreness induced by stair running. Another study by McGrath, Whitehead and Caine (2014) had mixed results. This study followed a similar protocol to Chen et al (2011) as it compared static stretching, PNF and a no-stretch CON. Neither stretching protocol provided any relief for muscle pain associated with DOMS. Pain scores were inadvertently higher post stretching in the PNF group. It is possible this was caused by further strain (from the stretch), which aggravated a fatigued muscle (McGrath et al., 2014). The additional strain may have initiated an inflammatory response, consequently increasing symptoms of DOMS (McGrath et al., 2014). With regard to static stretching, some participants described less pain following the recovery period. However this only occurred if there had been an increase in muscle length. Individual differences between participants were attributed to this occurrence (McGrath et al., 2014). A factor that may have contributed to these conflicting results was the time dedicated to each study. Chen et al (2011) ran their study over an eight-week period, thus providing extra time for the stretching protocols to take effect and encourage physical changes in muscle tissue. This suggests that consistent long term stretching, as opposed to single sessions, could lessen the pain associated with DOMS, therefore questioning its effect on acute recovery (McGrath et al., 2014; Chen et al., 2009).

Two studies that have looked at stretching in comparison to other recovery methods, and followed similar research designs, compared stretching to other post-match recovery methods in team sport athletes. Kinugsa and Kilding (2009) compared seven minutes of static stretching to nine minutes of CWT and a nine-minute combined protocol of CWI and active recovery. Throughout the duration of the one-week study, participants played three 90 minute football (soccer) games. Explosive leg power, HR, body temperature and perceived recovery were all measured before and after each game, after recovery and 24 hours post-recovery (Kinugsa \& Kilding, 2009). In comparison, Montgomery, Pyne, Hopkins, Dorman, Cook and Minahan (2008) compared lower body stretching (along with carbohydrate consumption) with CWI and 
lower body compression garments during a three-day simulated basketball tournament. All participants completed performance tests to determine baseline values. These included the Yoyo intermittent recovery test, vertical jump, 20 metre sprint and a basketball specific agility drill. Similarly to Kinugsa and Kilding (2009) the participants then played three 48-minute basketball games over the course of three days. Every day participants completed fatigue and soreness scores, as well as a vertical jump test. The performance tests were repeated on day four (Montgomery et al., 2008). Both studies produced similar results as stretching did not appear to enhance subsequent performances, despite the addition of carbohydrate in Montgomery et al (2008).

A feature of multiple stretching studies, as shown in Kinugsa and Kilding (2009) and Montgomery et al (2008), is the addition of other recovery methods in the investigations. These studies allow the comparison of a relatively simple method with more elaborate methods, as well as explore the prospect of combining recovery methods. While both Montgomery et al (2008) and Kinugsa and Kilding (2009) were not supportive of stretching, the combination of stretching and massage may also have potential. This was investigated by Delextrat, Hippocrate, Leddington-Wright and Clarke (2014), who carried out a study involving a group of elite basketball players. Participants received a massage, massage and stretching or rested passively following a competitive game. Vertical jump and repeat shuttle run sprints were used to assess subsequent performance, in combination with perceived fatigue and muscle soreness. Massage and stretching appeared to be more effective at improving recovery, compared to massage. While both massage groups increased vertical jump height, only massage and stretching improved repeated shuttle runs and perceived recovery measures. As both stretching and massage have the potential to reduce swelling and pain to aid muscle function, it appears combining two methods may intensify this effect (Delextrat et al., 2014). Previous research has found static stretching may be detrimental to subsequent performance, particularly in maximal force production (Cramer, Housh, Johnson, Miller, Coburn \& Beck, 2004), running speed and reaction times (Winchester, Nelson, Landin, Young \& Schexnayder, 2005), factors which are arguably important for performance tests such as vertical jump and repeated shuttle runs. This circumstance may have been a contributing factor to the results obtained by Montgomery et al (2008) and Kinugsa and Kilding (2009) as both these studies included measures of power and speed. With regard to Delextrat et al (2014), the researchers theorised that there may be a relationship with the stretch-shortening cycle (SSC) (also known as a countermovement or prestretch action). Stretching is thought to aid the series elastic components of the muscle, which 
store potential energy, and the spinal reflexes required for the SSC. This would appear to contradict Cramer et al (2004) and Winchester et al (2005), yet Delextrat et al (2014) believe applying massage to those structures after stretching may prove to be a determining factor. Muscle tissue may become more compliant, increasing ROM and enabling athletes to perform specific movement patterns efficiently (Young, 2007). Multiple reviews have each made conclusions supporting both sides of the argument. For example, Behm and Chaouachi (2011) concluded that static stretches held for 30 seconds or less at a low intensity may not cause power decrements. Interestingly, the stretching times implemented by Delextrat et al (2014) exceeded this. Meanwhile, Simic, Sarabon and Markovic (2013) determined that static stretching is likely to cause losses of strength and power. It is clear these conflicting views justify further investigation.

A potential issue for many of these stretching investigations is the measurement of the stretch intensity. While Kruse et al (2016) used joint angles as a way to quantify different stretching intensities, other studies have used subjective measures such as the pain and discomfort experienced during the stretch (Sands, McNeal, Murray, Ramsey, Sato, Mizuguchi \& Stone, 2013). Meanwhile, neither Montgomery et al (2008), Kinugsa and Kilding (2009) and Delextrat et al (2014) disclosed the stretching intensities used in their studies. Also, the use of different muscle groups in studies can make comparing results difficult as athletes with diverse sporting backgrounds will likely possess variations in flexibility and ROM, as well as perceptions of pain (Sands et al., 2013).

\section{Napping}

Sleep is widely considered to be necessary for overall health and well-being, as well as athletic performance and recovery and is described as a "complex physiological and behavioural state" (Halson, 2013, p.1). In relation to athletic performance, the circadian rhythm (the process that controls the sleep-wake cycle) is thought to influence the release of testosterone and cortisol, two hormones that are influential in muscle growth. During periods of sleep deprivation the circadian rhythm can be disturbed, which can also affect the hormonal balance. Due to the anabolic (testosterone) and catabolic (cortisol) nature of these hormones, an imbalance could impair muscle growth and in the long-term cause atrophy (Dattilo, Antunes, Medeiros, Monico Neto, Souza, Tufik \& de Mello, 2011). As the focus of this review is acute recovery strategies, the following section will focus on napping, brief periods of sleep throughout the day (Halson, 2015). Napping has been identified as potentially beneficial, especially for athletes who are 
experiencing sleep loss or those who have early morning training sessions (Halson, 2015). Sleep deprivation is a common occurrence among the athletic population, particularly when factors such as international travel (and subsequent jet lag) and heavy competition schedules are considered. Late night training sessions and events are likely to cause higher arousal levels, making it harder for athletes to fall asleep (Fullagar, Duffield, Skorski, Coutts, Julian \& Meyer, 2015).

Due to the additional physical and psychological demands, athletes typically require more sleep than the average person. It is known that if the balance between the sleep-waking cycles and circadian rhythm are disrupted, performance decrements can occur (Reilly \& Edwards, 2007). Napping has been suggested as a method for alleviating reductions in performance caused by recurrent periods of sleep loss (Reilly \& Edwards). Waterhouse, Atkinson, Edwards and Reilly (2007) found increasing sleep length by adding day-time naps improved athlete performance, particularly sprint time. This study followed a crossover design, meaning participants were tested under both conditions (with and without a nap) one week apart. Following a 30-minute nap (or no nap), participants were tested on sprint performance, hand grip strength, reaction time and short-term memory. The main finding from this study was an improvement in sprint time following a 30-minute nap (Waterhouse et al., 2007). While the sprint distance was relatively short (20 metres), making the results difficult to apply to sprinting events covering greater distances, this could be applied to team sport athletes who are more likely to sprint this shorter distance. However an aspect not included in this study, which would be applicable to team sport athletes, is repeated sprint ability as only three sprints were performed with a oneminute rest between each sprint.

Davies, Graham and Chow (2010) also conducted a study investigating the effect of napping on recovery following endurance training. In contrast to previous studies evaluating 20 to 30 minute naps, this study tested the effect a 90-minute nap would have since slow wave sleep (SWS), also known as deep sleep, is more likely to occur during longer periods of sleep (Bonnar, Bartel, Kakoschke \& Lang, 2018). Currently, the relationship between sleep, recovery and performance is not fully understood but one theory suggests SWS promotes ideal conditions for cell growth and repair (Bonnar et al., 2018). In an attempt to consider circadian timing and determine if there is an optimal time for naps to occur, Davies et al (2010) required their participants to complete a 90-minute endurance session, followed by a nap at either 10.30am or 11.30am, either one or two hours after the endurance session. Subjective measures, 
including alertness and readiness to train were used to assess this. Polysomnography was used to measure sleep. There was a greater duration of SWS during the 11.30am nap compared to the 10.30am nap, indicating this time period is more compatible with circadian timing. In terms of the subjective measures, the 11.30am nap improved readiness to train, compared to the 10.30am nap, which possibly reflects the main finding.

In a similar study to Waterhouse et al (2007), Petit, Mougin, Bourdin, Tio and Haffen (2014) who examined the effect a 20-minute nap would have on subsequent sprint performance, this time using a cycle ergometer. The results contradict those produced by Waterhouse et al (2007) as a 20-minute nap did not improve subsequent sprint performance. These contrasting results could be the result of the mild sleep deprivation inflicted upon the participants in the Waterhouse et al (2007) study. It is also possible that 20 minutes is not long enough for SWS to occur, therefore potentially limiting the rate of cell growth and repair (Petit et al., 2014). A phenomenon similar to that of Waterhouse et al (2007) was reported by Blanchfield, LewisJones, Wignall, Roberts and Oliver (2018), who found napping improved endurance performance and increased time to exhaustion (TTE) in participants who had the least sleep the night before. Despite similar results to Waterhouse et al (2007) there was a slight difference in the sleep patterns of the Blanchfield et al (2018) participants. Waterhouse et al (2007) imposed a reduced sleep protocol the night before to guarantee sleep deprivation among the participants. In contrast Blanchfield et al (2018) did not so participants received their usual amount of sleep. This allowed Blanchfield et al (2018) to speculate that day-time naps may benefit athletes who naturally sleep less. For those of who do receive sufficient sleep, a day-time nap may not be required to improve subsequent performance.

Based on relatively recent research, Bonnar et al (2018) proposed the timing of naps could affect an athlete's psychological recovery, specifically their perceived feelings and motivation for subsequent sessions and events. Cognitive recovery appears to benefit from napping, which may affect factors such as reaction times, decision making and skill execution, as observed by Waterhouse et al (2007). Blanchfield et al (2018) included RPE in their study, which were lower in participants who had improved their subsequent TTE performance following a nap. This indicates napping may have reduced the participants' sense of effort, therefore enabling them to improve their TTE. 
Previously discussed studies have focused on napping in a training environment, rather than a competitive setting. In fact, current knowledge is based upon anecdotal evidence, rather than scientific study. This is understandable considering athletes preferring to have as little interruptions as possible while preparing for a competitive event (O'Donnell, Beaven \& Driller, 2018). O'Donnell et al (2018) provided some preliminary evidence on the effect match day napping can have on elite netballers. When naps were limited to less than 20 minutes, improvements in countermovement jump (CMJ) height and perceived performance were noted. However as this was a field-based study, nap times were managed by the participants themselves, resulting in little consistency and the risk of outside variables influencing results. Despite this, these findings support the work of Waterhouse et al (2007) in terms of physical performance, and Blanchfield et al (2018) regarding perceived measures.

In order for a day-time nap to be effective there appears to be certain criteria that must be adhered to (Waterhouse et al., 2007). In order to manage the effects of SWS, naps shorter than 20 minutes (Waterhouse et al., 2007), or 90 minutes (Davies et al., 2010) are cited as providing the most benefits (O’Donnell et al., 2018). Durations longer or shorter are thought to cause sleep inertia, a state which can be detrimental to performance by briefly reducing arousal, alertness and fine motor skills (Hilditch, Dorrian \& Banks, 2017). Twenty minutes is deemed short enough to prevent waking during SWS, otherwise 90 minutes is considered long enough to allow a full sleep cycle to take place before waking (Hilditch et al., 2017).

\section{Nutrition}

The primary goals of recovery nutrition are the replenishment of muscle and liver glycogen, replacing lost fluids and electrolytes and ingesting adequate protein to promote muscle growth and repair (Beck, Thomson, Swift \& von Hurst, 2015). The importance of these goals is dependent upon many factors, especially the demands of the sport and the length of the training session. For example, a marathon runner and a weightlifter are unlikely to follow the same nutritional strategies (Beck et al., 2015). Replenishing muscle and liver glycogen as soon as possible was initially thought to be of particular importance. A very early study found postponing carbohydrate intake by two hours after exercise decreased the rate of glycogen synthesis (Ivy, Katz, Cutler, Sherman \& Coyle, 1988). This finding has been queried as more recent research has found this to only be necessary when multiple sessions or events take place in a single day (Burke, Hawley, Wong \& Jeukendrup, 2011; Parkin, Carey, Martin, Stojanovska \& Febbraio, 1997). In fact, when the time between sessions is less than eight hours, it is 
recommended that carbohydrates are consumed as soon as possible. This is particularly relevant to endurance athletes who often deplete their glycogen stores during training and events (Burke et al., 2011). It has been established within the literature that 1.0-1.2 $\mathrm{g}$ carbohydrate/kg body weight, consumed immediately following the first session, and then every hour for four hours afterwards is adequate for quick glycogen replenishment (Burke et al., 2011). An important point to consider is while these recommendations have been promoted for intermittent/anaerobic based sports, the foundation of the supporting research is based upon endurance sport (Ivy et al., 1988; Kovacs, 2006; Mujika \& Burke, 2010). The physical demands of endurance and intermittent based sports, including team sports, varies considerably. Endurance athletes usually move in one direction, often performing repetitive movement patterns at constant speeds. Running and cycling are typical examples. In contrast, most team sports include multiple short sprints and changes of direction. This places more demand on the glycolytic and ATP-PCr systems and may influence the type of nutritional strategies required for recovery (Williams \& Rollo, 2015). Overall, there are fewer studies on glycogen replenishment in team sport environments. A common feature among these studies is their focus on single sports and the specific performance outcomes for that sport. Incidentally, a large proportion of this research has focused on football (soccer) players (Williams \& Rollo, 2015). Early research found the process of glycogen replenishment after team sports took longer. This was initially attributed to extra eccentric muscle contractions associated with stopgo movements and collisions between players (Asp, Daugaard, Kristiansen, Kiens \& Richter, 1998). This has since been supported by various studies. Zehnder, Muelli, Buchli, Kuehne and Boutellier (2004) found carbohydrate consumption after sprinting activity had no effect on glycogen resynthesis. Surprisingly, glycogen also decreased during the two-hour post exercise period. Simultaneously, increases in inorganic phosphate and phosphocreatine (PCr) were also evident, which peaked during the two-hour post exercise period. The ratio of inorganic phosphate and PCr has been described as a reliable measure of muscle damage (Lund, Vestergaard-Poulsen, Kanstrup \& Sejrsen, 1998), thus possibly signifying the presence of EIMD. Zehnder et al (2004) determined that the carbohydrates consumed after exercise may have been used to fuel the regeneration of hydrolised ATP (which inorganic phosphate is a product of), which was prioritised over muscle glycogen replenishment. Krustrup, Ortenblad, Nielsen, Nybo, Gunnarsson, Iaia, Madsen, Stephens, Greenhaff and Bangsbo (2011) also found glycogen resynthesis to take over 24 hours in a group of elite football players, despite consuming carbohydrate 30 and 60 minutes post-match. The decrease in muscle force and increase in muscle soreness indicated the presence of DOMS. Following a similar thought 
process as Zehnder et al (2004), muscle damage sustained during football match play has been cited as a possible reason for this. These results imply carbohydrate cannot diminish this effect, thus questioning the recommendations regarding post exercise carbohydrates for team sport athletes (Zehnder et al., 2004).

Due to the intermittent and physical nature of many team sports, combined with frequent eccentric contractions, there is little doubt that protein is needed to repair the damage inflicted upon muscles. (Ozmen et al., 2014; Beck et al., 2015). This process causes a negative protein balance, due to the increase in protein turnover and can only be reversed through protein consumption (Beck at al., 2015). However there is some confusion regarding the timing of protein ingestion (Beck et al., 2015). According to some researchers, there appears to be an optimal window for protein intake immediately following exercise, known as an anabolic window (Aragon \& Schoenfeld, 2013). It has been suggested that amino acid uptake peaks following exercise. The resulting increase in amino acid concentration may also increase protein synthesis (Levenhagen, Carr, Carlson, Maron, Borel \& Flakoll, 2002; Kimball \& Jefferson, 2004). Levenhagen, Gresham, Carlson, Maron, Borel and Flakoll (2001) provided evidence to support this through a within subject study. When the participants in their study consumed a supplement immediately after exercise, protein synthesis rates tripled. Yet when the same supplement was consumed three hours after exercise, protein synthesis only increased by 12 percent. This finding has been disproved. Tipton, Rasmussen, Miller, Wolf, OwensStovall, Petrini and Wolfe (2001) compared muscle protein synthesis rates with pre and postexercise protein intake. Based on their results Tipton et al (2001) concluded that pre-exercise protein intake promoted a higher rate of protein synthesis. They believed the stimulation of protein synthesis, initiated by protein intake, combined with increased blood flow (exercise induced) improved amino acid delivery to muscle tissue. Furthermore, a meta - analysis by Aragon and Schoenfeld (2013) concluded that priority should be given to overall protein intake, rather than the timing of intake. This meta-analysis also questioned the reliability of the anabolic window hypothesis as it is based on the assumption that exercise is performed fasted. In a fasted condition negative protein balance will increase due to exercise induced muscle protein breakdown (Kumar, Atherton, Smith and Rennie, 2009). As a result, any protein consumed immediately after training will undoubtedly increase protein synthesis (Aragon and Schoenfeld, 2013). This raises doubt of the applicability of this hypothesis as many athletes do not train in a fasted state. 
There is also some evidence that combining protein and carbohydrate in a recovery meal can accelerate muscle protein synthesis. Chocolate milk has been recognised as being an ideal recovery food. It contains similar amounts of carbohydrate as specially formulated sports drinks and also protein, making it ideal for glycogen and protein resynthesis (Jentjens \& Jeukendrup, 2003). Spaccarotella and Walter (2011) compared two contrasting beverages (chocolate milk and a carbohydrate-electrolyte drink) for recovery in between morning and afternoon training sessions, in collegiate level football players. Using a shuttle run to fatigue test, which was completed following the afternoon training sessions, the results indicated that chocolate milk provided similar benefits as the carbohydrate-electrolyte drink as there was little difference in time to fatigue following the consumption of both drinks. The researchers believe the mixture of carbohydrates, protein and electrolytes in a liquid form could accelerate recovery by initiating glycogen resynthesis (Spaccarotella and Walter, 2011). Slightly different results were produced by Thomas, Morris and Stevenson (2009), who found cyclists who consumed chocolate milk in between endurance cycling bouts were able to cycle further than those who consumed carbohydrate replacement or fluid replacement drinks. This study highlights the main difference between a carbohydrate-electrolyte drink and chocolate milk, which is the higher fat content of the latter. As aerobic exercise predominantly uses fat as fuel, chocolate milk could reduce the speed of glycogen depletion, and thus improve endurance capacity (Thomas et al., 2009). However for intermittent activity performed at a greater intensity, like team sports, this may not be applicable because of the higher need of carbohydrate during anaerobic exercise (Spaccarotella and Walter, 2011). Nevertheless, according to Cockburn, Bell and Stevenson (2013) consuming milk after team sport activity could minimise exercise induced muscle damage. Despite there being no improvement in physiological measures (CK and myoglobin) or perceived muscle soreness, sprint and agility performance showed some promise. Theoretically, consuming carbohydrate and protein after exercise could alleviate the effect muscle damage seems to have on glycogen resynthesis, as reported by Zehnder et al (2004) and Krustrup et al (2011). Based on the results of Gunnarsson, Bendiksen, Bischoff, Christensen, Lesivig, Madsen, Stephens, Greenhaff, Krustrup and Bangsbo (2013) this may not be the case. Glycogen replenishment was not enhanced by a carbohydrate and protein rich recovery meal following a football match. This slightly contradicts Spaccarotella and Walter (2011) but this could have been influenced by the exercise setting. Both recruited football players, yet Spaccarotella and Walter (2011) used training sessions as their exercise protocol, while Gunnarsson et al (2013) used competitive and simulated matches. It is feasible that participants may have worked harder, and at a higher intensity during match play, possibly 
resulting in additional fatigue and muscle damage. Nevertheless, this insinuates that post exercise carbohydrate and protein intake is dependent on a multitude of factors (Williams \& Rollo, 2015).

Proper hydration is essential for both athletic performance and general health. Many physiological functions, such as metabolic processes (energy production), thermoregulation, maintenance of blood pressure (BP) and muscle contraction require fluid (Kenney et al., 2012). During exercise, metabolic heat production increases, leading to increases in muscle and core temperature. The production and evaporation of sweat occurs, in order to prevent overheating. When fluid lost through perspiration is not replaced dehydration can occur. A consequence of dehydration can be a reduction in blood volume, which may place additional strain in the cardiovascular system, thus leading to decreases in both physical and cognitive performance (Kenney et al., 2012). Volume and composition of fluid are important factors that need to be considered during the post-exercise rehydration process, and can be affected by individual sweat rates, temperature and time spent exercising (Kenney et al., 2012).

To retain fluid balance after exercise, relying on water is not recommended. Ideally, drinks used for rehydration should contain electrolytes, such as sodium and potassium which also need to be replaced after perspiration (Evans, James, Shirreffs \& Maughan, 2017). A consequence of drinking large amounts of pure water is a decrease in plasma sodium concentration and osmolality, which can actually cause a negative fluid balance through diuresis (Peake, 2019). Multiple studies have demonstrated the benefits of including sodium and potassium in post-exercise drinks to aid rehydration. It is generally accepted that an inverse relationship exists between the sodium concentration of the drink and the volume of urine produced, meaning a positive fluid balance is more likely (Evans et al., 2017). This relationship cannot be applied to potassium. The inclusion of an additional electrolyte, chloride in some of the potassium focused studies has added confusion when interpreting results (Evans et al., 2017). For example, the drinks investigated by Perez-Idarraga and Aragon-Vargas (2014) contained chloride and appeared to increase rehydration compared to water. Meanwhile, Shirreffs, Aragon-Vargas, Keil, Love and Phillips (2007) reported little difference between a potassium-rich drink, sodium-rich drink and bottled mineral water, which contained no chloride. 
Despite the accepted theory that rehydration is necessary for subsequent performance, research in the area has revealed some inconsistencies (Merson, Maughan \& Shirreffs, 2008). During their study, Merson et al (2008) compared rehydration after exercise using four beverages containing various sodium concentrations ranging from 0 to $50 \mathrm{mmol} / \mathrm{L}$. It was apparent that the beverages containing 40 and $50 \mathrm{mmol} / \mathrm{L}$ of sodium allowed for greater fluid retention and rehydration. Despite this, there was no difference in subsequent exercise performance compared to the first exercise bout, regardless of the beverage consumed. Based on this and other reviews, the extent of performance decrements may be affected by the speed at which dehydration occurs, the type of exercise, environmental conditions and participant's core temperature (Merson et al., 2008; Sawka \& Noakes 2007). In saying that, Sawka and Noakes (2007) raise a valid point when interpreting research in this area, with regards to research design. Most studies are conducted in a laboratory setting in which participants are required to execute tasks they may not perform in a real-life setting. A classic example of this is performing a two-hour basketball session in the heat while limiting fluid intake, behaviour unlikely to be replicated during a real training session, yet this was the protocol employed by Dougherty, Baker, Chow and Kenney (2006). Additionally, asking participants, especially high-level athletes, to commence exercise with limited fluid may affect their willingness to fully participate (Sawka \& Noakes, 2007). A study by Dugas, Oosthuizen, Tucker and Noakes (2006) supports this claim. Participants who were informed of limited fluid intakes exercised at a lower intensity. When not informed of this information, exercise intensity increased. Since this was observed from the beginning of the exercise bout, a behavioural change was suspected in which participants reacted in anticipation of the unpleasant experience of exercising with limited fluids.

For wheelchair athletes, especially those with a SCI, fluid replacement methods used by ablebodied athletes are not always appropriate or practical (Goosey-Tolfrey, Paulson \& GrahamPaulson, 2016). Able bodied athletes are able to adjust their fluid intake based on their sweat rate and fluid loss during exercise. Body weight measurement before and after exercise is a simple method to estimate fluid loss (Sawka, Burke, Eichner, Maughan, Montain, \& Stachenfeld. 2007). Athletes with a SCI are not able to regulate their body temperature as effectively and have reduced sweating capacity. Environmental conditions and the site of the SCI will also influence this, particularly for athletes with an injury further up the spinal cord, making fluid replacement difficult to calculate (Goosey-Tolfrey et al., 2016). There is evidence that consuming cold fluids may reduce the thermal strain associated with exercise (Lee \& 
Shirreffs, 2007). However, there is a potential disadvantage of this, an example of which was demonstrated by Goosey-Tolfrey, Krempien and Price (2014). Case studies carried out by these researchers aimed to determine voluntary fluid intakes of wheelchair athletes with paraplegia and quadriplegia. Fluid intakes of athletes with paraplegia are comparable to able-bodied athletes. However for athletes with quadriplegia, whose sweating ability is further limited, their fluid intake was much higher (Goosey-Tolfrey et al., 2014). This response is likely due to greater body temperature, and their attempt to alleviate it (Price \& Campbell, 2003). Consequently, athletes with quadriplegia are more likely to gain weight during exercise, which could be counterproductive to performance as additional mass will require more effort to propel around the court (Goosey-Tolfrey et al., 2014). This may increase reliance on other recovery methods such as CWI to reduce thermal strain (Goosey-Toldrey et al., 2016). Research on the benefit of specialised sports drinks for athletes with a disability, particularly those with a SCI, is limited. Anecdotal evidence suggests they are just as useful, especially after long and intense training sessions, or endurance events when glycogen needs to be replaced (Goosey-Tolfrey et al., 2016).

\section{Compression Garments}

Compression garments are tight fitting items of clothing with an inbuilt compression gradient, and range from upper, lower and full body options. Historically compression garments have been used in medical settings for patients with circulatory and vascular disorders (Duffeild \& Portus, 2007). Their popularity has increased within the athletic population for use during and after training and competition because of the belief that they aid both performance and recovery (Barnett, 2006). There is research to support the use of compression garments, in particular their influence on the inflammatory processes associated with EIMD. The pressure exerted by the garments may reduce the osmotic pressure and subsequently limit the space available for swelling, as well as preventing pain (Hill, Howaston, van Someren, Leeder \& Pedlar, 2014). Improvements in blood flow, venous return and waste product removal are also potential mechanisms thought to enhance recovery (Hill et al., 2014).

Goto and Morishima (2014) examined the effects of wearing a whole-body compression garment for 24 hours after resistance exercises performed at 70 percent one repetition maximum (1RM). Measures of muscular strength (1RM and maximal voluntary contraction), blood analysis, arm and thigh circumference and perceived muscle soreness were used to assess 
recovery. Recovery of upper and lower body muscular strength was significantly quicker after compression garment use. Muscle soreness was also lower. These findings may correlate with the arm and thigh circumferences as only the CON group experienced increases in girth measurements, indicating very little swelling occurred when the compression garments were worn. An additional finding was the accelerated recovery of the upper body, which took 3-8 hours, in comparison to the lower body which took the full 24 hours (Goto \& Morishima, 2014). A similar result was also present in another study investigating whole-body compression garments (Kraemer, Flanagan, Comstock, Fragala, Earp, Dunn-Lewis, Ho, Thomas, SolomonHill, Penwell, Powell, Wolf, Volek, Denegar \& Maresh, 2010). The upper body musculature also demonstrated superior recovery as bench throw performance improved dramatically following compression garment use. With regards to the lower body, ultrasound detected lower levels of swelling in the compression garment group. However this did not translate to improved performance, which was assessed through CMJ and squat jumps (Kraemer et al., 2010).

The results from Goto and Morishima (2014) and Kraemer et al (2010) align with those of a meta-analysis by Brown, Gissane, Howatson, van Someren, Pedlar and Hill (2017) which found compression garments enhanced recovery from strength-based sports more effectively than recovery from endurance and power based sports. Resistance training, especially when high loads have been prescribed, has been shown to produce higher levels of CK compared to endurance activities like running and cycling, indicating higher amounts of muscle damage has occurred (Brown et al., 2017). Of the strength-based studies included in their review, those that measured markers of muscle damage described inverse relationships between reductions in $\mathrm{CK}$ and improvements in strength recovery, with Kraemer et al (2010) being one of those studies. Brown et al (2017) also found compression garments provided considerable benefits to strength recovery during the initial eight hours following resistance training, as demonstrated by Goto and Morishima (2014). A finding that was not understood was the similar levels of muscle damage and $\mathrm{BLa}$ in the compression garment and $\mathrm{CON}$ groups, even though strength recovery was significantly faster in the compression garment group (Goto and Morishima, 2014). A similar finding was also reported by Jakeman, Byrne and Eston (2010) who found participants wearing a compression garment improved strength, squat and CMJ performance, but made no difference to CK concentration. 
With regards to power recovery, Brown et al (2017) argued that when muscle damage is combined with the additional recovery demands required following power-based activity (such as metabolic by-products and neurological fatigue) compression garments are not sufficient to accelerate the recovery process. After further investigation, results differ. As previously mentioned, Jakeman et al (2010) revealed an improvement in squat and CMJ performance. Additionally Rugg and Sternlicht (2013) also noted increases in CMJ height when participants wore lower body compression garments, in comparison to loose fitting shorts. It was hypothesised that the compression garment may increase proprioception as the stretchy and supportive nature of the garment fabric may provide a recoil effect, which may support the wearer's ability to produce and maintain power output (Rugg and Sternlicht, 2013). Meanwhile Duffield, Cannon and King (2010) challenged these results as their study reported the effects compression garments had on recovery following sprint and plyometric activity to be trivial. Furthermore, physiological measures of lactate, CK or CRP proved to be no different to the CON group, suggesting there are some conditions in which compression garments make no difference to physical performance.

The fact that some of the studies discussed have uncovered improvements in performance measures, yet little to no change in physiological measures highlights the potential of a placebo effect. This has been investigated and described by Hill et al (2014). This study involved marathon runners using either lower body compression tights or a false ultrasound treatment. Despite there being little difference in measures of muscle damage, inflammation and muscular strength between the two groups, participants using the compression garments reported improved subjective measures as perceived muscle soreness was lower 24 hours after the marathon run. Pruscino, Halson and Hargreaves (2013) described similar findings in a group of hockey players following simulated match play. After the fatiguing exercise, CK and CRP were elevated and power output decreased in both groups, yet perceived recovery was significantly higher in participants who wore the compression garments. In fact participants in the CON group reported higher muscle soreness at every time point, with it peaking for both CON and EXP one hour post-exercise. Curiously CK concentration did not peak until 24 hours post-exercise. This disparity between muscle soreness and CK insinuates that muscle soreness may not be a direct measure of muscle damage (Pruscino et al., 2013). Without discounting muscle soreness as a valid measure, there is evidence that muscle soreness can indicate the conditions within the muscle and subjective measures such as readiness for exercise (Nosaka, Newton \& Sacco, 2002). 
Comparing data from compression garment studies can be difficult due to the variation in pressure gradients, as some studies do not disclose this information or have determined pressures via manufacturer instructions (Brown et al., 2017). Pressure gradients can also be influenced by how they fit on various body shapes so researchers would need to consider anthropometric measurements. This would certainly make it difficult to maintain consistent pressure gradients, which would arguably be a critical component when determining the effectiveness of compression garments (Brown et al., 2017).

\section{Vascular Occlusion}

Vascular occlusion is a technique whereby blood flow to chosen limbs is temporarily restricted by a blood pressure cuff type device (Northey, Rattray, Argus, Etxebarria \& Driller, 2016). This technique has been used to preserve cardiac and skeletal muscle tissue following an ischaemic reperfusion injury, which occurs when blood flow returns after a prolonged period of restriction (Page, Swan \& Patterson, 2017). This type of injury is considered comparable to muscle damage following exercise, leading to the belief that vascular occlusion could improve athletic recovery (Page et al., 2017). When the cuff is released the resulting vasodilation and increase in adenosine concentration appears to improve blood flow, which aids nutrient delivery and waste removal (Northey et al., 2016). An alternative theory describes a reduced inflammatory response, specifically. Reduced muscle oedema and intramuscular pressure limit nocioreceptor response (also known as pain receptors), which could influence muscle soreness (Howatson \& van Someren, 2008). ATP sensitive potassium channels are also activated, which may improve skeletal muscle contractility potentially activating a muscle pump action and supporting blood flow and venous return (Northey et al., 2016).

One of the first studies to focus on vascular occlusion in an exercise setting was carried out by Beaven, Cook, Kilduff, Drawer and Gill (2012). Pressure cuffs were applied to both legs for two three-minute intervals, immediately after a lower-body exercise session. The same exercise session was repeated immediately post and 24 hours later. Vascular occlusion appeared to provide some benefits to jump height and repeated sprint ability. Mean squat jump height improved, however eccentric peak velocity and peak acceleration during the CMJ and peak power output during the squat jump decreased immediately afterwards. However after 24 hours the adverse effects on power output and velocity were reversed as peak concentric and eccentric velocity improved. Page et al (2017) is one of the few other studies to reveal some benefit of 
vascular occlusion as a recovery technique. Following a strength-based exercise protocol to induce muscle damage, participants who received vascular occlusion treatment recovered and exhibited pre-exercise muscular strength levels 24 hours earlier than the CON group. In contrast, a small number of known studies have refuted the findings of Beaven et al (2012) and Page et al (2017) with results which are not supportive of vascular occlusion as a recovery technique. Between them, these studies recruited athletes from a range of disciplines, specifically rugby (Garcia, Ribeiro da Mota, Scott Leicht \& Marocolo, 2017), strength training (Northey et al., 2016), sprint cycling and middle-distance running (Borne, Hausswirth \& Bieuzen, 2016). A possible reason for the inconsistencies in the results may be the methodologies used by the researchers, with particular reference to the vascular occlusion protocol. Some studies applied intermittent pressure (Beaven et al., 2012); Northey et al., 2016), compared to Borne et al (2016) whose participants continuously wore the cuffs for 24 minutes, albeit at a much lower pressure. Interestingly, apart from Borne et al (2016), the other studies applied standardised cuff pressures $(220 \mathrm{mmHg})$. Without meaning to, this may have created additional methodological differences between the studies. Using the same pressure without considering participant anthropometric measurements, particularly girth measurements, may have caused inconsistencies in the actual pressures exerted on individual participants (Loenneke, Allen, Mouser, Thiebaud, Kim, Abe \& Bemben, 2015). Various factors have been considered in an effort to account for this, such as limb composition and limb circumference (Loenneke, Thiebaud, Fahs, Rossow, Abe \& Bemben, 2013). One such method involves plotting thigh circumference with arterial occlusion pressure (AOP) for each participant and applying a pressure of 60 percent of each participant's AOP. Specific measurements and pressures were formulated based on this process. They include $<45-50 \mathrm{~cm}=$ $120 \mathrm{mmHg} ; 51-55 \mathrm{~cm}=150 \mathrm{mmHg} ; 56-59 \mathrm{~cm}=180 \mathrm{mmHg}$ and $\geq 60 \mathrm{~cm}=210 \mathrm{mmHg}$ (Loenneke et al., 2013). Although, the applicability of these measurements has only been trialled using the lower body, so their usefulness for upper body vascular occlusion, and therefore wheelchair athletes is unknown (Loenneke et al., 2013).

A recent study on vascular occlusion by Williams, Russell, Cook and Kilduff (2018), took the methods of Loenneke et al (2013) into consideration when performing their study, by utilising individualised cuff pressures based on limb circumference. CMJ, blood (BLa, CK) and saliva (cortisol, testosterone) analysis were used to assess physiological and neuromuscular recovery following a sprint-based session. Vascular occlusion made no difference to these measures, indicating recovery was not enhanced. This contradicts the findings of Beaven et al (2012) and 
Page et al (2017), possibly due to the training status of the participants included in these studies, as both these studies recruited healthy, active individuals, compared to the well-trained rugby players recruited by Williams et al (2018). Even so, inter-individual variability must be considered as there is evidence to suggest differences in EIMD responses exist depending on gender, age and ethnicity (Damas, Nosaka, Libardi, Chen \& Ugrinowitsch, 2016). This has even become apparent when participants perform the same exercise, leading to suggestions that vascular occlusion may only benefit some athletes/individuals (Damas et al., 2016; Williams et al., 2018).

\section{Intermittent Sequential Pneumatic Compression}

Intermittent sequential pneumatic compression (ISPC) is a device that inflates to a specified level of pressure while worn on the athlete's arms or legs. ISPC can provide up to five times more pressure than a typical compression garment (Mizuno, Arai, Todoko, Yamada \& Goto, 2017). ISPC was initially developed to treat and prevent conditions such as lymphedema, deep vein thrombosis (DVT) and pulmonary embolisms by increasing blood circulation (Chen, Frangos, Kilaru \& Sumpio, 2001). As the device intermittently inflates and deflates, the pressure gradient improves venous return and decreases the risk of blood clots forming (Chen et al., 2001). In terms of athlete recovery, improving blood flow increases oxygen transportation to the muscles. Theoretically, this maximises nutrient delivery and the removal of waste products that have accumulated during training or competition (Winke \& Williamson, 2018). Current theories are based on evidence that ISPC can reduce oedema, pain and fatigue (Winke \& Williamson, 2018; Martin, Friedenreich, Borges \& Roberts, 2015; Sands, McNeal, Murray \& Stone, 2015).

In comparison to the other recovery methods discussed, (hydrotherapy, stretching, nutrition, massage, compression garments and vascular occlusion), ISPC is relatively new in the sport setting and has not received the same attention within the literature (Martin et al., 2015). The few studies which have investigated the efficacy of ISPC have reported potential benefits in alleviating muscle soreness (Winke \& Williamson, 2018; Sands et al., 2015), reducing swelling (Winke \& Williamson, 2018) and improving BLa clearance (Martin et al., 2015).

Winke and Williamson (2018) compared 20 minutes of ISPC treatment with a continuously worn compression sleeve over a five-day period following an exercise session. The session consisted of isokinetic concentric elbow flexion and eccentric elbow extension exercises. By 
measuring ROM, muscle swelling (upper and lower arm girth measurements) and pain before and every day after the exercise protocol the researchers found ISPC significantly improved the speed at which recovery occurred. All measures of the participants who used ISPC were consistently lower and returned to baseline levels approximately a day quicker (Winke \& Williamson, 2018).

Martin et al (2015) and Overmayer and Driller (2018) completed similar studies looking at recovery using an ISPC device and subsequent performance. BLa was used as a measure in both studies. Neither study discovered any performance benefits yet Martin et al (2015) found lactate clearance improved considerably after participants had used ISPC. Overmayer and Driller (2018) found ISPC had little effect on BLa. These contrasting results may have been influenced by the participants from the two studies. While the participants in Martin et al (2015) study were described as active and healthy individuals, the participants recruited by Overmayer and Driller (2018) were elite, well-trained cyclists.

Similarly to Martin et al (2015), the participants involved in the Winke and Williamson (2018) study were not considered elite but recreationally fit. Despite using alternative measures, both produced similar results with ISPC positively influencing recovery and reducing overall recovery time. However, Winke and Williamson (2018) did not use any physiological indicators to assess recovery. Muscle swelling was measured using bicep circumference and ROM was measured using a goniometer. Interestingly, one of the methods to measure pain was palpation by the researcher rather than a tool, such as an algometer (Despite the researcher's best intention to apply the same pressure when palpating the muscle, this could be subjected to user error (Winke \& Williamson, 2018). A device such as an algometer is a reliable piece of equipment that is used extensively in clinical settings (Park, Kim, Park, Kim \& Jang, 2011).

Sands et al (2015) looked specifically at muscle soreness, however unlike Winke \& Williamson (2018), they used an algometer to assess PPT. This study produced results in favour of ISPC as athletes experienced a greater pain threshold following the ISPC use in between morning and afternoon training sessions, while those in the control group showed no changes. A key, and potentially relevant difference between Sands et al (2015) and many of the other studies included in this review is the variation in sports represented by the participants. They ranged from power and strength dominant (weightlifting) to endurance dominant (triathlon). Additionally, the exercise session completed by the participants before the recovery period 
involved their own sport training. This meant the researchers were unable to control the type, duration and intensity of sessions, which would likely have resulted in participants experiencing varying levels of fatigue. The use of PPT as a measurement was an attempt by the researchers to account for this as selecting a performance test to measure sport specific muscle soreness was found to be problematic. Other options such as changes in maximal force or task failure tests were unlikely to objectively assess the demands of all the sports represented, so the validity of the results may have been questionable (Sands et al., 2015).

Hanson, Stetter, Li and Thomas (2013) recruited a group of high-level college athletes. Similarly, to the previous studies discussed, this study used a cycle ergometer protocol and BLa to assess ISPC. A variable added to this study, unlike previous studies, was the inclusion of an active recovery group. While there was no significant difference between the active recovery and the ISPC groups, they were both more effective at reducing BLa compared with passive recovery (Hanson et al., 2013). However, a possible limitation of this study, is the anaerobic element of the cycle ergometer protocol. This only lasted a single minute, significantly shorter than the protocols performed in Martin et al (2015) and Overmayer and Driller (2018), therefore questioning whether the participants were sufficiently fatigued. Ensuring participants are adequately fatigued before commencing the recovery protocol is more likely to result in an impartial assessment of the recovery techniques. Without sufficient fatigue, it is unlikely the capacity of the technique(s) under investigation will be fully understood (Hanson et al., 2013).

Meanwhile Marcello, Fortini and Greer (2019) also used HR and BLa as measures to test ISPC. However rather than using a high intensity protocol, such as the Wingate test, their participants performed two 60-minute steady state cycle rides at 60 percent of threshold power. The 30minute recovery period took place in between these two rides and consisted of either ISPC or passive recovery in a supine position. HR and BLa were measured at regular intervals throughout both rides. No differences were found in HR between the CON and ISPC groups. The most surprising result was an increase in BLa in the ISPC group during the second ride. The researchers were unsure if this was due to an increase in lactate production or if lactate clearance was compromised (Marcello et al., 2019). These results contradict the findings of Martin et al (2015). Differences in session length and intensity of the exercise bouts may have caused these conflicting results, or, and potentially more likely, differences in BLa sampling. 
Unlike the majority of previous studies, Marcello et al (2019) did not measure BLa during the recovery period, instead choosing to do so 30 minutes into both cycling sessions.

As discussed previously, depleted glycogen levels often occur following hard exercise and can subsequently influence performance in further training sessions. In one of the first to explore this area, Keck, Cuddy, Hailes, Dumke and Ruby (2015) investigated how ISPC may influence muscle glycogen replenishment following 90 minutes of cycling with the aim of depleting glycogen. BLa, insulin and $\mathrm{VO}_{2}$ peak were measured, along with muscle biopsies for glycogen analysis. The researchers hypothesised that increases in blood flow associated with ISPC may also improve muscle glucose uptake and subsequently muscle glycogen (Keck et al., 2015). Despite this no differences in glycogen replenishment rates were found between the CON group (passive recovery) and the ISPC group. A factor that should be considered was the provision of carbohydrate recovery drinks for all participants. An increase in plasma blood glucose and insulin after the post-exercise carbohydrate drink indicated glucose was available to be metabolised by the muscles. Even though blood flow was not measured directly, there was little change in HR which may show ISPC did not improve blood flow. This finding is similar to the studies which found no effect on lactate clearance so related physiological processes, especially those which influence blood flow, may be involved (Keck et al., 2015; Martin et al., 2015; Overmayer \& Driller, 2018). Therefore the researchers concluded that it was the ingestion of carbohydrates immediately post-exercise that influenced glycogen synthesis rather than ISPC (Keck et al., 2015). Repeating this trial with the addition of a second cycle ride following recovery may provide some insight as to whether carbohydrate intake and ISPC can influence subsequent performance.

When comparing ISPC studies, the type of compression needs to be considered. A peristaltic compression device, as used by Sands et al (2015) and Martin et al (2015), inflates and deflates separate compartments independently. In contrast, the compartments in a sequential compression device inflate in a sequential manner then deflate as one (Winke \& Williamson, 2018; Ovemayer \& Driller, 2018). It has been suggested that peristaltic compression bears more resemblance to natural muscle pump, possibly making it more effective (Hanson et al., 2013). However, based on the studies in this review, it is unclear whether peristaltic or sequential is the superior technique. The sequence and timing of pressure exerted on the muscles is different, making it possible for muscle soreness, oedema and blood flow to be affected in different ways (Lim, Kim, Lee, Seo \& Ko, 2018). 
Currently the length of time participants spend using the ISPC devices ranges from 20 to 30 minutes, with 30 second inflation deflation cycles. On average maximum pressures of 80 $\mathrm{mmHg}$ are prescribed in most studies. Keck et al (2015) implemented a slightly different protocol of two 60-minute ISPC treatments with a maximum pressure of $90 \mathrm{mmHg}$ separated by one hour. Alternatively, longer inflation deflation cycles of 50 seconds has been cited as providing additional benefits, especially in relation to fluid mobilisation (Zaleska, Olszewski, Jain, Gogia, Rekha, Mishra \& Durlik, 2013).

\section{The Placebo Effect}

An athlete's perception of various recovery techniques may be influenced by the placebo effect. In the sports science field, there are a multitude of studies investigating various performance enhancing techniques, which have highlighted the influence of a placebo effect (Halson \& Martin, 2013). Based on this, it could be argued that belief alone can result in improvements in performance. This was initially thought to be a relatively simple phenomenon, which was used in medical studies to determine the true efficacy of various treatments (Levine, Gordon \& Fields, 1978). Levine et al (1978) were one of the first to study this area, specifically looking at pain and analgesia. Based on this research, along with further studies, there are now suggestions that neurobiological processes are operating (Halson \& Martin, 2013). Including a placebo as part of a study design encourages the participants' belief in the technique being investigated. Therefore, the participants' expectation of the technique and anticipation of its success may become influencing factors. When combined with other factors such as the athletes' previous experiences and preconceived beliefs, reducing the influence of potential bias is improbable. This is especially applicable when the technique in question is the most current and state of the art (Halson \& Martin, 2013). It is plausible that some of the studies discussed in this review have been influenced by the placebo effect, which usually presents itself through improvements in performance and subjective feelings, with little to no change in physiological measures. Due to the way in which they are applied, compression garments and nutrition interventions in particular are often subject to this type of bias. For studies focusing on other methods, including a placebo is slightly more problematic and often requires an element of deception. For example, it has been cited that a flaw of hydrotherapy research is the lack of studies with a placebo condition (Broatch, Petersen \& Bishop, 2014). Broatch et al (2014) compared CWI and CON groups with a placebo trial. Deception was needed to convince 
participants of the supposed benefits of the placebo trial. In an attempt to fully assess the extent to the placebo effect, participants were required to complete a questionnaire designed to measure their belief in the effectiveness of their assigned group. According to the results, participants in the placebo trial reported more belief in the technique, improved subjective feelings of pain and readiness to exercise, compared to the CON trial. There was also evidence of enhanced physical recovery. Participants in the placebo trial also displayed improved recovery of muscular strength and power, similar to those in the CWI group.

\section{Exercise Recovery in Athletes with a Disability}

Since the Paralympic movement began, participation rates have rapidly increased in many Paralympic sports with wheelchair basketball and wheelchair rugby becoming two of the most popular (Paulson \& Goosey-Tolfrey, 2017). The standard of competition has also increased and there are more professional opportunities available for athletes with disabilities to train full time with the same support and resources available to able bodied athletes (Paulson \& GooseyTolfrey, 2017). Unfortunately, research in this area is limited, resulting in this research including research involving athletes with a range of disabilities.

Research has investigated the physiological, biomechanical and performance elements of disability sport. Quite often theories and concepts applied to training able bodied athletes are applied when training athletes with disabilities as well (Paulson \& Goosey-Tolfrey, 2017). While this has its merits, it is not always appropriate. Anecdotal evidence has its limitations, particularly as the range of physical disabilities represented in disability sport is extensive and affects every athlete differently (Paulson \& Goosey-Tolfrey, 2017). An example of this is the use of HR monitoring during training (Goosey-Tolfrey \& Price, 2010). During exercise, the sympathetic nervous system increases HR. The specific nerves that control this mechanism are found in the lumbar and thoracic regions of the spinal cord. Therefore, wheelchair athletes who have sustained a SCI may not be able to increase their HR above a certain point as the site of a spinal cord lesion will affect the functionality of these nerves (Goosey-Tolfrey \& Price, 2010). Paraplegia occurs when damage is sustained in the lumbar and sacral areas of the spinal cord so only the legs and torso (to varying severities) are affected (Theisen, 2012). Quadriplegia (also known as tetraplegia) occurs when the spinal cord is damaged further up the spinal cord, in the thoracic and cervical areas. This results in limited arm function, along with lower limb 
paralysis (Theisen, 2012). Blood flow can also be compromised, which will ultimately affect the distribution of oxygenated blood to the working muscles (Theisen, 2012).

The quantity of research focusing on recovery methods for athletes with disabilities seems to be even more limited, particularly when compared with able bodied athletes (Paulson \& Goosey-Tolfrey, 2017). This was noticeable while searching the literature as a high proportion of participants were able bodied. There is evidence that injury rates among Paralympic athletes may be higher than able bodied athletes (Fagher \& Lexell, 2014). This has been further supported by Fagher, Jacobsson, Timpka, Dahlstrom and Lexell (2016), who believe Paralympic athletes may be more susceptible to illness and injury because of underlying conditions related to their disability. In fact, recovery may be of greater importance for athletes with disabilities, as neglecting this could increase the risk of injury, illness and OTS (Fagher et al., 2016).

\section{Monitoring Fatigue and Recovery in Athletes with a Disability}

The same tools used for assessing recovery in able bodied athletes (as discussed above) have received minimal attention within the literature. For example, Edmonds, Burkett, Leicht and McKean (2015) and Leicht, Bishop, Paulson, Griggs and Goosey-Tolfrey (2012) examined the use of salivary markers and heart rate variability (HRV) as monitoring tools in elite Paralympic swimmers and wheelchair athletes respectively, with both providing useful information. Subjective measures have also received some attention, particularly the measure of session rate of perceived exertion (sRPE) (Paulson, Mason, Rhodes \& Goosey-Tolfrey, 2015). Due to the limitations of HR, sRPE has been reported as a reliable alternative in both wheelchair basketball (Iturricastillo, Yanci, Granados \& Goosey-Tolfrey, 2016) and wheelchair rugby (Paulson et al., 2015). An important limitation to this research is the short training time frame in which the data was collected, especially when other research recommends athlete data should be collected over an extended period of time as it is more likely to provide accurate data (Bourdon et al., 2017).

The performance measures (discussed earlier) used for monitoring able bodied athlete performance are not always practical for athletes with disabilities. Specific testing has been developed, particularly for wheelchair athletes, which has been based on similar tests for able bodied athletes (Goosey-Tolfrey \& Leicht, 2013). Logistical limitations do exist, particularly around specialised equipment that is often required, meaning field testing is much more 
common compared to able bodied sport. Arguably, this may increase the accuracy of the result, making them applicable to real-life settings (Goosey-Tolfrey \& Leicht, 2013). Sprint (ranging from 5-20 metres) and agility drills that can be modified are considered valid and reliable measures of performance (Yanci, Granados, Otero, Badiola, Olasagasti, Bidaurrazaga-Letona, Iturricastillo \& Gill, 2015). Likewise, for those athletes who are unable to jump, the maximal medicine ball throw has been validated for assessing upper body power (DeGroot, Balvers, Kouwenhoven \& Jansses, 2012).

\section{Hydrotherapy}

Thermoregulatory processes are not as effective in athletes with disabilities, particularly those with a SCI. This must be taken into consideration, especially when training and competing in hot environments (Goosey-Tolfrey et al., 2016). As discussed previously, hydrotherapy techniques may provide relief from thermal strain and assist subsequent performance (Halson, 2011). Goosey-Tolfrey, Swainson, Boyd, Atkinson and Tolfrey (2008) performed a study to test the efficacy of hand cooling in improving subsequent performance in able bodied and wheelchair athletes after exercise induced increases in body temperature. Hand cooling was found to be effective at lowering the core temperature of both able bodied and wheelchair athletes, however this effect was not as pronounced in the wheelchair participants. In the able bodied participants, arteriovenous anastomoses (AVAs) in the hands are thought to have continued to dilate and allow the cooled blood to flow to the core (Goosey-Tolfrey et al., 2008; Walloe, 2016). As the wheelchair participants were not able to increase their body temperature to the same extent as able bodied participants, the researchers believe a phenomenon known as counter current heat exchange may have occurred. If body temperature remains within normal range, the AVAs may have vasoconstricted as a protective mechanism against the cold water, resulting in cooler blood being redirected to deeper veins so it can be warmed by arterial blood (Goosey-Tolfrey et al., 2008). Despite this, subsequent performance improved, and ratings of thermal sensation decreased, along with the psychological stress associated with training in warm temperatures. This indicates hand cooling may be used as a recurrent recovery technique, rather than pre-cooling, a popular technique among able bodied endurance athletes (Grahn, Cao $\&$ Heller, 2005). This is not evident in wheelchair athlete populations, a possible consequence of the physiological variations in wheelchair athletes. This further highlights the impracticalities of comparing able bodied and disabled athletes (Paulson \& Goosey-Tolfrey, 2017). 
Prystypa, Stefaniak and Rudenko (2017) investigated the effect that CWT (alternating Swiss shower) and massage had on HR and BP during recovery in powerlifters with CP. Curiously, when performed after training, CWT and massage produced opposing effects. Massage resulted in decreases in HR, systolic and diastolic BP. This was expected because of the enhanced blood flow associated with massage (Brukner \& Khan, 2012). This was also based on previously discussed studies such as Zadkhosh et al (2015), Ogai et al (2008) and Kennedy et al (2018), which highlighted possible psychological benefits of massage, a reduction in BP would seem logical. Yet following CWT treatment HR and systolic BP increased. This finding is surprising as both treatments are thought to increase blood flow, and theoretically lower BP (Brukner \& Khan, 2012; Pournot et al., 2011). Alternating between hot and cold water, as well as the subsequent vasodilation and vasoconstriction may have contributed to this increase. Prystypa et al (2017) believe this increased the load on the haemodynamics of the participants. It is difficult to compare the results of this study to others as they exact details of the hydrotherapy treatment were not included. Important information such as the time spent under hot and cold water or whether participants were sitting or standing when HR and BP were measured were not available.

\section{Massage}

Kennedy, Patil and Trilk (2018) only recently completed one of the first studies to investigate the benefits of massage therapy for Paralympic level cyclists. The aim of this study was to analyse how massage could influence sleep quality, pain, stress levels and functional ability (specifically muscle tightness and spasticity). The participants involved in this study had a range of physical disabilities, including SCI, CP, amputations and traumatic brain injuries. Despite this study not focusing on massage as an acute recovery method, one of the findings could be of interest in relation to this review. Massage was found to increase the participant's perception of recovery, as they felt able to train and compete with more intensity (Kennedy et al., 2018). Due to this study being completed in a real-life setting, external validity may be higher than studies completed in a laboratory setting, potentially increasing generalisability of the data (Camm \& Fox, 2018).

\section{Compression Garments}

Vaile, Stefanovic and Askew (2016) looked at how a lower limb compression garment influenced lower and upper limb blood flow in wheelchair rugby players. This is one of the few studies that has involved wheelchair athlete participants and compression garments. The 
results of this study were enlightening and produced encouraging results, which showed improvements in both upper body blood flow and subsequent performance. The researchers proposed a possible link between these findings, with increased blood flow to the active muscles a likely contributor to improved performance (Vaile et al., 2016). These results are different to a similar study, which found no performance benefits for wheelchair athletes while wearing compression garments (Rinaud, Calmels, Roche, Mongold, Trudeau \& Devillard, 2007). There are key differences which may have contributed to this. Vaile et al (2016) focused on sub-maximal exercise, while Rinaud et al (2007) looked at maximal exercise intensity. The compression garments used created similar pressure, but it is possible the relatively low pressure was inadequate for exercise at a higher intensity (Rinaud et al., 2007). The type of SCI may also have had an impact. The participants recruited by Vaile et al (2016) were quadriplegic while the participants recruited by Rinaud et al (2007) were paraplegic. As mentioned previously, the site of the injury and subsequent level of paralysis can influence factors such as blood flow and HR, which may have influenced the results of these studies (Theisen, 2012). An additional finding by Rinaud et al (2007) was a decrease in BLa following exercise when the compression garments were worn during and after the exercise bout. However, this was only found in the participants with lower level spinal cord lesions. This could be explained by the limited functional muscle mass of the participants with higher level spinal cord lesions, as they were simply not able to work at an intensity high enough to produce BLa (Bhambhani, 2002).

\section{Gaps in the Literature}

Based on this literature review, there is an abundance of research that has investigated numerous athletic recovery methods for able bodied athletes.

As well as the limited research on recovery in athletes with disabilities, ISPC research has predominantly focused on recovery of the lower body. Consequently, research on upper body recovery is limited, therefore highlighting a key gap in the literature. Winke and Williamson (2018) completed one of the few ISPC studies focusing entirely on upper body exercise recovery. The promising results obtained from this study suggest ISPC may enhance recovery from upper body dominant exercise, particularly from DOMS related symptoms. Perceived measures also appear to benefit from ISPC. However whether this relates to the novelty of this technique remains to be seen and requires additional research to provide further understanding. Nevertheless, there is theoretical evidence that ISPC has the potential to be an effective method 
of recovery in wheelchair athletes. Its proposed mechanisms for improving blood flow and metabolite removal may benefit those with impaired circulation, especially those with a SCI.

\section{Conclusion}

There is little doubt athletic recovery is essential, yet its multifaceted nature results in it being dependent upon the individual needs of the athlete and the demands of their sport. It seems, based on the literature discussed above, the use and effectiveness of these recovery techniques is dependent upon many factors, particularly the time available between sessions or events, the type of exercise (strength or endurance based), duration and intensity of exercise performed. Hydrotherapy techniques appear to provide some benefit in thermoregulation and reducing inflammation yet may inhibit sprint speed and power production, as well as reduce long term training adaptations (Halson, 2011). Active recovery has been cited as useful in enhancing BLa clearance but may inhibit glycogen replenishment (Neric et al., 2009; Fairchild et al., 2003). Research supporting massage report improvements in swelling, pain and BLa clearance, yet other research has described the benefits of massage to be purely psychological (Poppendieck et al., 2016). Stretching may also improve muscle pain and blood flow, however like hydrotherapy, may also reduce force production and power output (Kruse et al., 2016; Cramer et al., 2004). Napping is thought to assist with cognitive recovery, and hormone balance, enabling the body to remain in an anabolic state. However these benefits appear to be duration dependent (Waterhouse et al., 2007; Davies et al., 2010). Post-exercise nutrition is supported by a large amount of research, although many of the recommendations proposed (eg. Postexercise carbohydrate intake) are based upon endurance athlete studies, making it impractical to apply to team sport athletes (Ivy et al., 1988; Kovacs, 2006; Mujika \& Burke, 2010). Compression garments are thought to reduce inflammation and swelling associated with EIMD and are frequently worn by athletes during and after training/events. The placebo effect has been highlighted as a potential mechanism as studies have reported performance recovery, yet no differences in physiological measures (Hill et al., 2014). Research investigating vascular occlusion is promising but significantly more is needed to provide more conclusive evidence (Kilduff et al., 2012; Williams et al., 2018). Finally, like many of the other techniques discussed, ISPC may reduce swelling, pain and improve BLa clearance, but variability in research methodology within the literature adds confusion (Martin et al., 2015; Sands et al., 2015; Winke \& Williamson, 2018). 
Inconsistencies within the literature also add to the confusion and makes it difficult to compare studies. For example, many hydrotherapy studies use variations in water temperature and submersion durations (Halson, 2011). Likewise, questions regarding optimal active recovery and stretching intensities (Menzies et al., 2010; Sands et al., 2013), along with the ideal pressures exerted by compression garments and ISPC (Brown et al., 2017; Zaleska et al., 2013) have added to the confusion.

For wheelchair athletes, and athletes with different disabilities, additional variables must be considered, especially the physiological impact their disability has on their ability to recover from exercise. Theoretically, many of the recovery methods discussed assist with these physiological processes, such as blood flow and thermoregulation (Paulson \& Goosey-Tolfrey, 2017; Thiesen, 2012), meaning they should provide some benefits. The paucity of research in this area makes it difficult to draw to conclusions, so more research is needed to provide additional knowledge for athletes and coaches. 


\section{CHAPTER TWO - Original Study.}

Has been accepted for publication in the American Journal of Physical Medicine and Rehabilitation. 


\section{The use of upper-body intermittent, sequential, pneumatic compression arm-sleeves on recovery from exercise in wheelchair athletes}

Objective: The aim of the current study was to investigate the efficacy of an upper body intermittent sequential pneumatic compression (ISPC) device on recovery after wheelchair team sport activity. Design: Eleven well-trained wheelchair basketball and rugby athletes ( 8 male, 3 female, mean \pm SD age $=33 \pm 10$ years) performed a series of measures pre-exercise, post-exercise and post-recovery (grip strength, pressure-to-pain threshold (PPT), medicine ball throw, wheelchair sprints, repeated sprints). Subjective muscle soreness and fatigue measurements were taken in conjunction with performance tests and 24 hours post-exercise. Participants completed two recovery trials, separated by one week, of either passive recovery $(\mathrm{CON})$ or 20 minutes of wearing recovery armsleeves (ISPC) applied to both arms. Results: No statistically significant interactions were found between the recovery method and time for any measures $(\mathrm{p}>0.05)$. Effect size analysis revealed a moderate effect $(d=-0.67)$ for muscle fatigue immediately after ISPC recovery. A large effect $(d=-0.96)$ for muscle soreness was found 24 hours post-recovery in favour of ISPC. Conclusion: ISPC may provide some benefit for perceptual recovery measures immediately following and 24 hours after high intensity wheelchair activity with negligible effects on performance-recovery.

Keywords: disability; fatigue; blood lactate; dynamic compression

\section{What is Known}

Previous research has highlighted the importance of including recovery techniques to expedite physiological and psychological recovery in an athletic training programme. Underperformance, overtraining and injury are possible consequences of insufficient recovery for athletes. Recovery techniques such as hydrotherapy, active recovery and massage, have been investigated with mixed results.

\section{What is New}

This study explores a relatively new recovery technique known as intermittent sequential 
pneumatic compression (ISPC). This study involves wheelchair athletes, a population regularly underrepresented in athletic recovery research. The results of this study highlight the potential of ISPC to benefit perceived recovery for wheelchair athletes.

\section{Introduction}

Optimal athletic recovery methods expedite the process in returning the body to homeostasis, meaning all physiological processes return to pre-exercise levels (Barnett, 2006). Recovery strategies are employed by athletes to initiate the regeneration of damaged muscle tissue, removal of metabolic by-products and replenishment of energy stores to allow subsequent sessions to be performed at the optimal intensity to encourage adaptations and enhance the desired performance benefits (Kellmann, 2010). Efficient recovery is critical in tournament settings, particularly if multiple games/events are scheduled on a single day (Montgomery et al., 2008). Many recovery strategies have been assessed comprehensively in the research literature including hydrotherapy, active recovery, massage, stretching and compression garments (Tavares, Smith \& Driller, 2017). A recovery method yet to be addressed to the same extent is upper-body intermittent sequential pneumatic compression (ISPC).

ISPC is a device that inflates to a level of pressure while worn on the participant's arms or legs. These devices provide up to five times more pressure than a compression garment (Mizuno et al., 2017). ISPC was first developed to treat and prevent conditions such as lymphedema, deep vein thrombosis (DVT) and pulmonary embolisms by increasing blood circulation (Chen et al., 2001). As the device intermittently inflates and deflates, the pressure gradient improves venous return and decreases the risk of blood clots forming (Chen et al., 2001). Regarding athletic performance and recovery, improving blood flow increases oxygen transportation to the muscles, thus enhancing nutrient delivery and the removal of waste products that have accumulated during training (Winke \& Williamson, 2018). There is also some evidence that ISPC can alleviate oedema, pain and fatigue (Winke \& Williamson, 2018; Martin et al., 2015; Sands et al., 2015).

Due to ISPC being a relatively new technique in the sport setting, there has been a paucity of research on its effectiveness, especially when compared to other more established recovery methods (Martin et al., 2015). Some studies have highlighted potential benefits of ISPC, particularly in alleviating muscle soreness (Winke \& Williamson, 2018; Sands et al., 2015), 
improving lactate clearance (Martin et al., 2015) and reducing swelling (Winke \& Williamson, 2018).

Sands et al (2015) investigated ISPC for recovery when used between morning and afternoon training sessions using a single measure, PPT. The participants included 24 elite athletes (12 EXP, 12 CON) who used ISPC following training. The results showed ISPC to be effective in improving PPT immediately following the recovery period in comparison to passive recovery. The improvement in PPT continued throughout the day, including after the afternoon training session. Martin et al (2015) also found ISPC to be beneficial. Fourteen physically active individuals performed two 30 second Wingate cycle tests, separated by three minutes of pedalling at $70 \mathrm{rpm}$. Following a 30-minute recovery period, a third Wingate was completed. Compared to the control group, lactate clearance improved significantly following ISPC, however differences in cycling performance between the treatment groups was deemed trivial (Martin et al., 2015).

Overmayer and Driller (2018) have also shown ISPC to be ineffective in improving subsequent cycling performance. Their study involved two bouts of cycling on a cycle ergometer, separated by a 30-minute recovery period (ISPC or passive recovery). No difference was found in BLa between treatment groups and there was minimal improvement in subsequent cycling performance following ISPC use ( $p>0.05$ ). These results support the findings of Northey, Rattray, Argus, Etxebarria and Driller (2016) who demonstrated that ISPC was unable to improve recovery and subsequent performance in 12 strength trained participants following resistance exercise. Measures of lower body power (squat and CMJ height) following ISPC showed no improvement relative to the control group.

A key gap in the current ISPC research is that to date, it has only focused on the lower body. Studies that have concentrated exclusively on the upper body are lacking. Winke and Williamson (2018) compared 20 minutes of daily upper body ISPC with a continuously worn upper body compression garment over a five-day period in eight recreationally trained participants. Muscle swelling and pain of the elbow flexor muscles were used to measure symptoms of DOMS. Muscle soreness was induced in the arms using eccentric and concentric muscle contractions Participants in the ISPC group returned to baseline values quicker than those wearing the compression garment, indicating that upper body ISPC may accelerate the healing of muscle tissue following DOMS. This indicates there may be some benefit to upper body dominant sports where repeated bouts of exercise are common. 
Wheelchair basketball and wheelchair rugby are two team sports currently played by physically disabled athletes (Ozmen et al., 2014). Both sports require speed, power, endurance and strength, along with technical and tactical skills. Due to the high intensity and physical nature of these sports, recovery is critical for subsequent performance, especially throughout a multiday tournament, with very limited rest time (Montgomery et al., 2008).

The conflicting results on lower body ISPC use, combined with sparse research on upper body ISPC warrants further investigation on this recovery strategy. Therefore, the purpose of this study was to investigate the effectiveness of ISPC as an upper body recovery tool for wheelchair basketball and rugby athletes. This design was selected to simulate a wheelchair team sports tournament scenario, where two games are often played on the same day.

\section{Methods}

\section{Participants}

Eleven wheelchair athletes volunteered to take part in the current study ( 8 male, 3 female, mean \pm SD age $=32.7 \pm 9.7$ years, Table 1 ). The participant's disabilities included SCI, Spina Bifida and lower leg amputation. The participants in the study were recruited from regional wheelchair basketball and rugby teams (7 basketball, 4 rugby). All participants were regional and/or international level athletes. They took part in at least two weekly training sessions and weekend games at the time of the study. Participants provided their classification number, which specifies each player's level of function (Table 1). Wheelchair basketball has nine classes (0.5-4.5) (International Wheelchair Basketball Federation, 2014). Wheelchair rugby has seven classes (0.5-3.5) (International Wheelchair Rugby Federation, 2015). In both sports the lower the classifications correspond to lower levels of function. All participants were required to provide informed written consent before taking part (Appendix 1). Ethics approval was acquired from the National Human Disability Ethics Committee (see Appendix 2). 
Table 1. Participant characteristics and disability classifications

\begin{tabular}{lllll}
\hline Participant & Age & Sex & Disability & Classification \\
\hline 1 & 31 & Female & Spina Bifida & 1.5 Basketball \\
2 & 41 & Female & Spinal cord injury & N/A Rugby \\
3 & 47 & Female & Spinal cord injury & 1.5 Rugby \\
Female Mean \pm SD & $39.7 \pm 8.1$ & & & \\
\hline 4 & 22 & Male & Spinal cord injury & 4.0 Basketball \\
5 & 40 & Male & Spinal cord injury & 3.5 Basketball \\
6 & 21 & Male & Lower limb amputation & 4.5 Basketball \\
7 & 46 & Male & Spinal cord injury & 3.0 Basketball \\
8 & 32 & Male & Spina Bifida & 1.5 Basketball \\
9 & 21 & Male & Spinal cord injury & 2.0 Basketball \\
10 & 31 & Male & Spinal cord injury & 1.0 Rugby \\
11 & 35 & Male & Spinal cord injury & 2.0 Rugby \\
Male Mean \pm SD & $31 \pm 9$ & & & \\
\hline Overall Mean \pm SD & $\mathbf{3 3 . 4} \mathbf{9 . 5}$ & & & \\
\hline
\end{tabular}

\section{Study design}

This study implemented a randomised, counterbalanced, crossover design whereby participants completed two trials separated by one week; recovery using the ISPC device (ISPC) and passive recovery (CON). Following a warm-up, the participants completed the first set of performance and perceptual measures (listed below), to provide a point of reference for the ensuing tests. The measures were repeated immediately post the high intensity exercise session and immediately after the recovery intervention (Figure 2). Testing took place at the same time of day each week. This was to control for diurnal variation. 


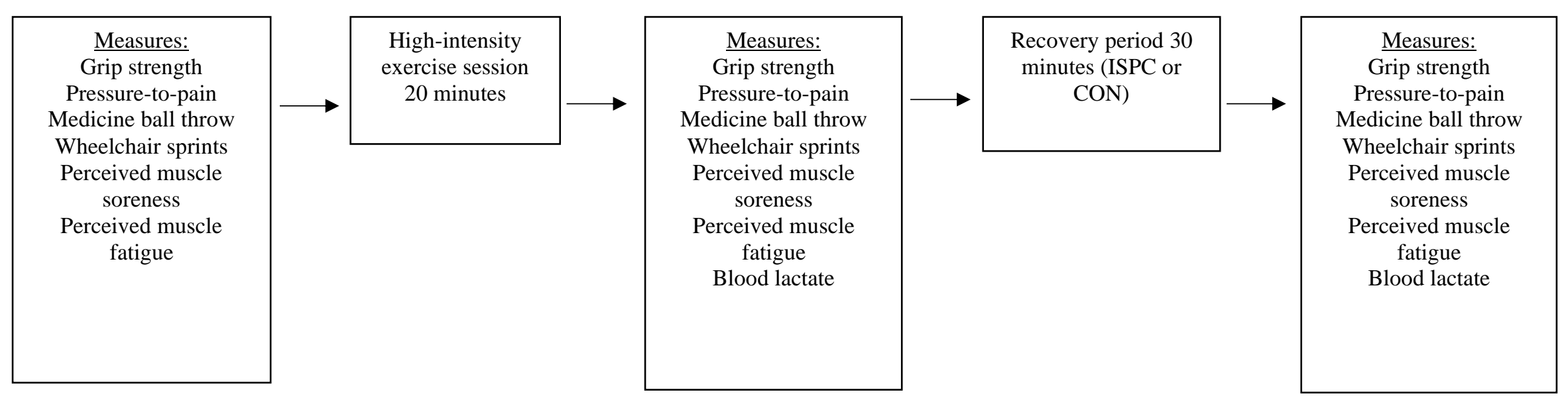

Figure 2. Study timeline 
The measures were selected because of their relevance and applicability to the demands of wheelchair team sports. They assess the skills and fitness components considered essential to wheelchair team sport performance (Ozmen et al., 2014). Before commencing, participants completed a warm-up consisting of five laps on a standard basketball court (28 x 15 metres). The performance measures included maximal medicine ball throw, repeated and single wheelchair sprints, grip strength and BLa. PPT was also measured along with perceptual measurements of muscle fatigue and soreness. All measures were completed three times, at the start, after the high intensity session and following the recovery period.

\section{Maximal medicine ball chest throw}

The maximal medicine ball throw measures upper body power, vital for passing and wheelchair propulsion (De Groot et al., 2012). The participants had three attempts to throw a 5-kilogram medicine ball as far as possible using the chest pass technique. The best throw was used for subsequent analysis. Each attempt was measured using a measuring tape taped to the floor and recorded in metres. Measurements were recorded to the nearest 10cm (De Groot et al., 2012).

\section{Wheelchair sprint and repeated sprints}

Wheelchair sprints were used to assess speed and acceleration. Timing lights (Speedlight Timing Gates, Swift Performance, Australia) were placed at five metre intervals, making a total sprint distance of 15 metres. This allowed five metre split times to be recorded. Three sprints, starting from a stationary position, were completed on a 30 second cycle. Participants had 30 seconds to complete each sprint and return to the start position ready to begin the next sprint. Times were recorded using the Speedlight Timing Systems app. The fastest time for each 5metre split and the total sprint time (sum of three sprints) was used for analysis.

\section{Grip strength}

The grip strength test measures isometric strength of the hand and forearm muscles. Hand and forearm strength are essential for skills in wheelchair-based ball sports, particularly wheelchair 
propulsion. A hand grip dynamometer was used (CAMRY, electronic hand dynamometer, model EH101, China) and gave a score measured in kilograms. Participants were instructed to keep their arm bent at a 90-degree angle and squeeze as hard as possible for approximately three seconds. Two attempts were made on each hand, alternating left and right hands with a 10 second rest in between. The best score on each side was used for analysis.

\section{Blood lactate}

Fingertip and ear lobe blood samples were collected using single-use lancets. The fingertip was the first choice; however, some participants with lower function wore gloves to aid in wheelchair propulsion. For those participants collecting blood samples from the ear lobe was more appropriate. BLa was measured using a Lactate Pro device (Lactate Pro2, Arkray, Japan). BLa was measured twice, immediately following the high intensity exercise session and immediately following the recovery period.

\section{Pressure-to-pain threshold}

A handheld algometer (FDN 100, Wagner Algometer, England) was used to measure PPT by applying pressure through a $11 \mathrm{~mm}$ rubber pressure pad. The algometer had a capacity of 100 N. PPT was measured at two anatomical sites, the right biceps brachii (Figure 3a) and right extensor digitorum (Figure 3b). To ensure consistency, both sites were marked using a highlighter pen at the beginning of the testing session. The algometer was operated as per the manufacturer's guidelines. Pressure was applied by the researcher at a speed of $10 \mathrm{~N} / \mathrm{s}^{-1}$, with one hand used to stabilise the arm. Participants were required to immediately alert the researcher when the sensation changed from pressure to pain (Broderick, Uiga \& Driller, 2019). 

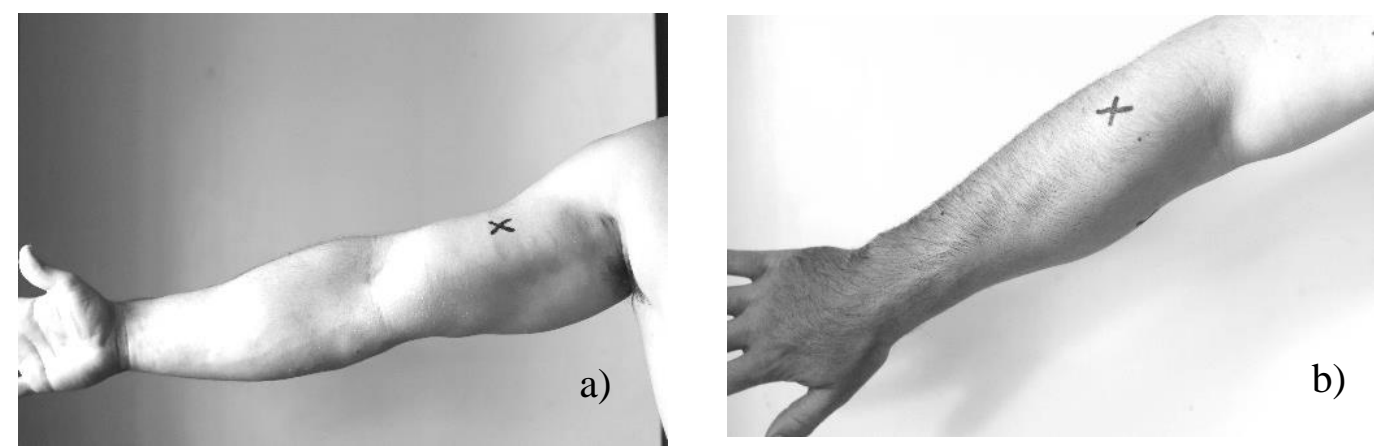

Figure 3. Landmarks for measurement of pressure-to-pain threshold using the algometer a) biceps brachii, and; b) extensor digitorum.

\section{Muscle soreness and fatigue scales}

Participants were asked to rate their muscle soreness and fatigue using a 0-10 scale with 0 equalling no soreness or fatigue and 10 corresponding with extreme soreness or fatigue (Montgomery \& Hopkins, 2013). Participants were also asked to provide scores 24 hours after the high intensity session. This was carried out via text message.

\section{High intensity exercise session}

The aim of the high intensity exercise session was to induce fatigue, similar to that experienced during a wheelchair basketball or rugby match. This consisted of three parts and required the participants to work at a high intensity with very short recovery periods (two minutes) between exercise bouts. The first drill consisted of 10 wheelchair court sprints (10 x 28 metres). Participants were allowed a brief recovery (no more than 10 seconds) as they wheeled back to the start of each sprint. The second drill was a figure of 8 agility drill. This required the participants to travel as fast as they could around two cones 6.5 metres apart in a figure of 8 formation. Participants did this continuously for 10 x 30 second rounds, with a 15 second rest period in between each round. The final drill consisted of a basketball court sprint (28 metres) immediately followed by three medicine ball chest throws. This was completed 10 times. The average recovery time between each sprint was approximately 10 seconds. Water was permitted ad-libitum during the rest periods. 


\section{Recovery treatment}

The recovery period incorporated two recovery protocols. During the CON protocol participants were instructed to rest passively for 30 minutes while remaining seated in their wheelchairs. The participants following the ISPC protocol wore the upper-body ISPC device (Recovery Pump Arm Sleeves, LLC, USA) for 20 minutes. To ensure the participants had the same recovery time, 10 minutes was allowed for setting up and removing the sleeves. The recovery sleeves were set to a pressure of $80 \mathrm{mmHg}$ with an inflation time of 30 seconds and deflation time of 15 seconds. The participant using the sleeves had their arms resting in an abducted position at approximately waist height. Two chairs positioned on either side of the participant were used to achieve this positioning (Figure 4).

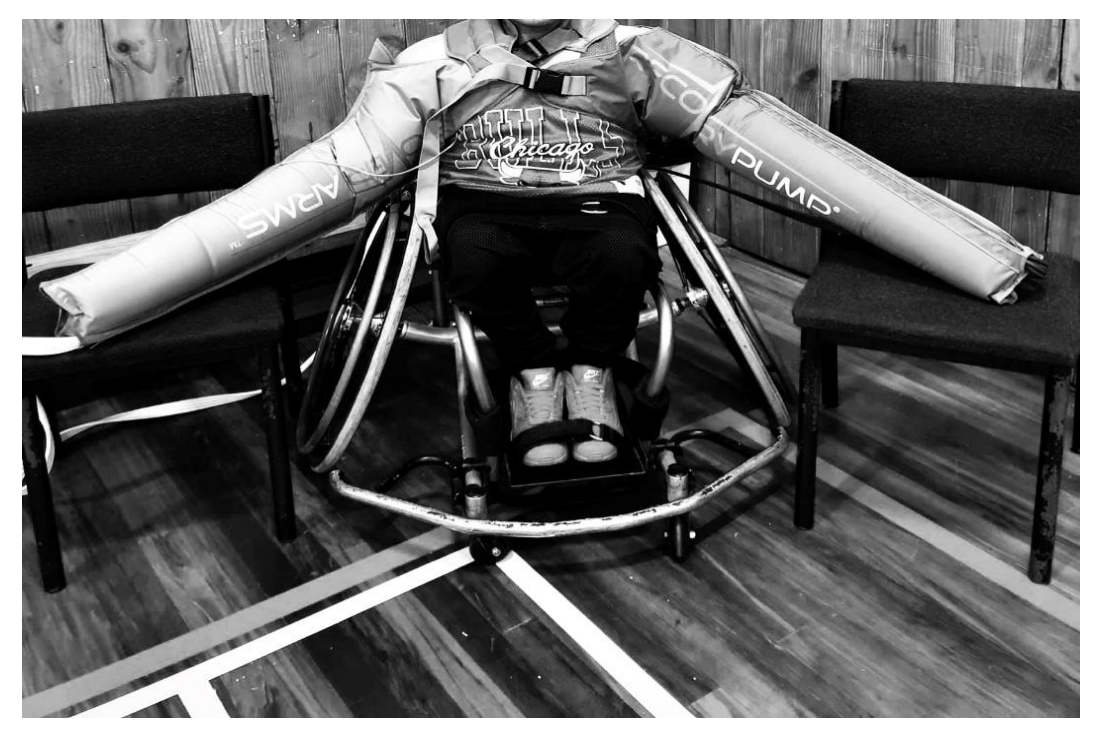

Figure 4. Positioning during intermittent sequential pneumatic compression (ISPC) recovery

\section{Pressure monitoring}

The Kikuhime device (Medi-Group, Australia) was used during the recovery period. This device is considered a valid and reliable tool for measuring the pressure gradients of compression garments (Brophy-Williams, Driller, Halson, Fell \& Kitic, 2014). The sensor was taped to the participant's right bicep brachii, the same site used for the muscle algometer measure, while their arm was inside the recovery sleeve. A pressure reading was recorded 
during each inflation cycle. The maximum pressure reached during the 20 -minute recovery period was used for analysis.

\section{Statistical analysis}

Statistical analysis was completed using the Statistical Package for Social Science (SPSS 25.0 IBM Corp, Armonk, NY, USA). A two-way repeated measures ANOVA was used to determine whether any statistically significant $(\mathrm{p}<0.05)$ two-way interactions existed between the two recovery methods (CON and ISPC) at various time points (pre-exercise, post-exercise, postrecovery and 24 hours post). A Bonferroni adjustment was made where significance was established. The Shapiro-Wilk test of normality was used to determine if the data was normally distributed. Mauchly's Test of Sphericity was used to determine whether the assumption of sphericity had been violated. For the measures that did violate the assumption of sphericity, the Greenhouse-Geisser estimate was used.

Analysis was also carried out to determine effect size using standardised (Cohen's $d$ ) effects. The threshold of each effect size was established using the following descriptors; 0.2 (small), 0.5 (moderate) and 0.8 (large). If the 90\% confidence intervals overlapped both positive and negative values the effect size was deemed unclear (Cohen, 1988).

\section{Results}

The mean pressure applied at the biceps brachii landmark by the ISPC device, measured using the Kikuhime device, was $76 \pm 5 \mathrm{mmHg}$.

The two-way repeated measures ANOVA revealed no statistically significant two-way interactions between recovery method and time for any measures (Table 2). Consequently, investigation of the main effects for both time and all measures were conducted individually. The main effect of time showed there to be statistically significant differences in muscle soreness $(\mathrm{p}=.002)$, muscle fatigue $(\mathrm{p}=.003)$, forearm algometer $(\mathrm{p}=.048)$, right hand grip $(\mathrm{p}=.011), 5 \mathrm{~m}$ sprint $(\mathrm{p}=.025), 15 \mathrm{~m}$ sprint, $(\mathrm{p}=.001)$, total sprint time $(\mathrm{p}=.002)$ and lactate $(\mathrm{p}<.001)$ at different time points. This was explored further using Bonferroni post hoc tests and Pairwise comparisons. There was a decrease in muscle fatigue from $5.7 \pm 1.8$ at the post- 
exercise stage to $3.0 \pm 2.9$ post-recovery following ISPC use (Figure 5a). Similarly, there was also a decrease in muscle soreness from $4.8 \pm 1.6$ at the post-exercise stage to $2.9 \pm 1.924$ hours post-exercise following ISPC use (Figure 5b). 
Table 2: Comparison of intermittent sequential pneumatic compression (ISPC) and control (CON) interventions at all time points. Data is presented as means \pm SD.

\begin{tabular}{|c|c|c|c|c|c|c|c|c|}
\hline & \multicolumn{2}{|l|}{ Pre-exercise } & \multicolumn{2}{|l|}{ Post-exercise } & \multicolumn{2}{|l|}{ Post-recovery } & \multicolumn{2}{|c|}{24 hours post } \\
\hline & ISPC & $\mathrm{CON}$ & ISPC & $\mathrm{CON}$ & ISPC & $\mathrm{CON}$ & ISPC & $\mathrm{CON}$ \\
\hline Muscle soreness (0-10) & $2.0 \pm 1.9$ & $2.5 \pm 1.6$ & $4.8 \pm 1.6$ & $4.6 \pm 1.4$ & $3.1 \pm 2.3$ & $3.6 \pm 1.8$ & $2.9 \pm 1.9$ & $4.2 \pm 1.9$ \\
\hline Muscle fatigue $(0-10)$ & $1.8 \pm 1.9$ & $1.9 \pm 1.4$ & $5.7 \pm 1.8$ & $5.4 \pm 1.8$ & $3.0 \pm 2.9$ & $4.1 \pm 1.9$ & $3.0 \pm 2.8$ & $3.5 \pm 2.9$ \\
\hline Grip strength left (kg) & $31.6 \pm 23.7$ & $32.1 \pm 24.1$ & $31.5 \pm 23.7$ & $32.7 \pm 24.4$ & $31.4 \pm 23.4$ & $32.3 \pm 24.4$ & - & - \\
\hline Grip strength right $(\mathrm{kg})$ & $29.8 \pm 23.9$ & $32.2 \pm 25.6$ & $30.4 \pm 24.7$ & $31.6 \pm 26.2$ & $28.9 \pm 23.1$ & $30.7 \pm 25.1$ & - & - \\
\hline Algometer forearm $(\mathrm{N})$ & $74.4 \pm 27.6$ & $70.7 \pm 26.4$ & $66.2 \pm 29.9$ & $59.1 \pm 32.3$ & $65.3 \pm 29.2$ & $57.1 \pm 32.9$ & - & - \\
\hline Algometer bicep (N) & $55.3 \pm 28.5$ & $60.5 \pm 27.8$ & $56.3 \pm 34.6$ & $55.1 \pm 35.0$ & $51.6 \pm 33.1$ & $53.6 \pm 37.0$ & - & - \\
\hline $\begin{array}{l}\text { Medicine ball throw } \\
\text { (m) }\end{array}$ & $3.25 \pm 1.39$ & $3.34 \pm 1.50$ & $3.24 \pm 1.42$ & $3.35 \pm 1.45$ & $3.50 \pm 1.35$ & $3.23 \pm 1.41$ & - & - \\
\hline $\begin{array}{l}\text { Wheelchair sprint - } \\
5 \mathrm{~m}(\mathrm{sec})\end{array}$ & $2.05 \pm 0.42$ & $2.09 \pm 0.46$ & $2.11 \pm 0.41$ & $2.17 \pm 0.49$ & $2.13 \pm 0.4$ & $2.12 \pm 0.44$ & - & - \\
\hline $\begin{array}{l}\text { Wheelchair sprint - } \\
10 \mathrm{~m}(\mathrm{sec})\end{array}$ & $3.66 \pm 0.81$ & $3.73 \pm 0.86$ & $3.75 \pm 0.76$ & $3.80 \pm 0.85$ & $3.81 \pm 0.80$ & $3.89 \pm 0.82$ & - & - \\
\hline $\begin{array}{l}\text { Wheelchair sprint - } \\
15 \mathrm{~m}(\mathrm{sec})\end{array}$ & $5.14 \pm 1.16$ & $5.26 \pm 1.27$ & $5.29 \pm 1.10$ & $5.35 \pm 1.25$ & $5.31 \pm 1.13$ & $5.39 \pm 1.33$ & - & - \\
\hline $\begin{array}{l}\text { Wheelchair repeated } \\
\text { sprint }(\mathrm{sec})\end{array}$ & $15.92 \pm 3.56$ & $16.22 \pm 4.09$ & $16.21 \pm 3.50$ & $16.31 \pm 4.01$ & $16.32 \pm 3.59$ & $16.53 \pm 4.30$ & - & - \\
\hline $\begin{array}{l}\text { Blood } \\
(\mathrm{mmol} / \mathrm{L})\end{array}$ & - & - & $9.1 \pm 5.7$ & $9.1 \pm 4.8$ & $5.0 \pm 4.4$ & $4.3 \pm 3.0$ & - & - \\
\hline
\end{tabular}



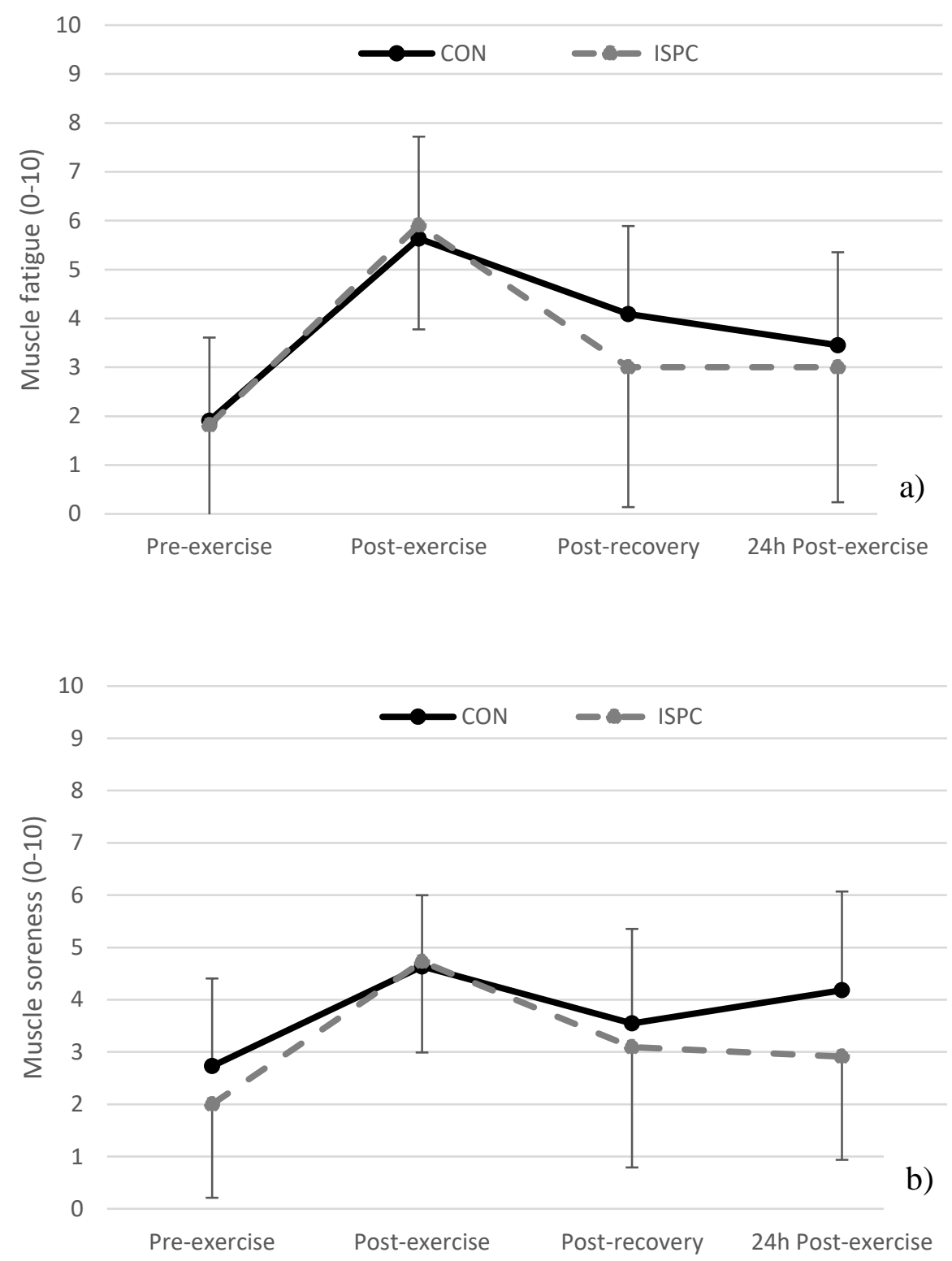

Figure 5. Comparison of intermittent sequential pneumatic compression (ISPC) and control (CON) interventions across time points for a) perceived muscle fatigue, and; b) perceived muscle soreness.

The effect size analysis (Table 3 ) between post-exercise and post-recovery showed ISPC contributed to moderate benefits for perceived muscle fatigue when compared to CON ( $d$ $=-0.67)$. The comparison of 24 hours to post-exercise showed large benefits for muscle soreness $(d=-0.96)$ and small benefits for muscle fatigue $(d=-0.44)$, in favour of 
ISPC. There were also small benefits for the medicine ball throw $(d=0.26)$ between postexercise and post-recovery for ISPC when compared to CON. 
Table 3. Comparison of all measures (post-recovery and $24 \mathrm{~h}$ post-exercise) compared to postexercise (pre-recovery) values. Data presented as raw difference in values (mean $\pm 90 \%$ confidence intervals) with Cohen's $d$ effect sizes (and 90\% confidence intervals) for comparison between intermittent sequential pneumatic compression (ISPC) and control (CON) interventions.

Post-exercise - Post-recovery

$\Delta$ ISPC $-\triangle \mathrm{CON}$

Effect size $\pm 90 \%$ CI
Post-exercise -24 hours-postexercise

$\Delta \mathrm{ISPC}-\Delta \mathrm{CON}$

Effect size $\pm 90 \% \mathrm{Cl}$

$-1.5 \pm 0.9$

$-0.96 \pm 0.57$, Large

$-0.8 \pm 1.1$

$-0.44 \pm 0.60$, Small

Grip strength dynamometer - left (kg)

Grip strength dynamometer - right $(\mathrm{kg})$

Algometer forearm (N)

Algometer bicep (N)

Medicine ball throw (m)

Wheelchair sprint $-5 \mathrm{~m}(\mathrm{sec})$

Wheelchair sprint $-10 \mathrm{~m}$ (sec)

Wheelchair sprint $-15 \mathrm{~m}(\mathrm{sec})$

Wheelchair repeated sprint (sec)

Blood lactate (mmol/L)
$0.3 \pm 1.9$

$0.01 \pm 0.08$, Trivial

$-1.2 \pm 1.3$
$-0.67 \pm 0.72$, Moderate

$0.6 \pm 1.2$

$-0.02 \pm 0.05$, Trivial

$1.1 \pm 5.2$

$0.03 \pm 0.16$, Trivial

$-3.2 \pm 8.8$

$-0.09 \pm 0.25$, Trivial

$0.40 \pm 0.30$

$0.26 \pm 0.22$, Small

$0.10 \pm 0.10$

$0.16 \pm 0.21$, Trivial

$0.0 \pm 0.10$

$0.04 \pm 0.11$, Trivial

$0.0 \pm 0.10$

$-0.02 \pm 0.09$, Trivial

$-0.10 \pm 0.40$

$-0.03 \pm 0.10$, Trivial

$0.2 \pm 2.2$

$0.04 \pm 0.52$, Unclear 


\section{Discussion}

The main finding in the current study was that upper body ISPC did not have a significant effect on performance recovery following a high intensity exercise session in wheelchair basketball and wheelchair rugby athletes. There was a small increase in medicine ball throw following recovery, however this difference was not statistically significant ( $p$ > 0.05). A secondary finding was that muscle fatigue and soreness scores decreased immediately following recovery and 24 hours post-exercise in the ISPC trial when compared to CON, thus indicating ISPC could have a positive effect on an athlete's perceived feelings of fatigue and soreness.

Regarding physical performance, the results from the current study are consistent with Martin et al (2015), Overmayer and Driller (2018) and Northey et al (2016) who also found ISPC to be ineffective in improving subsequent performance recovery. Sufficient fatigue is required to objectively evaluate ISPC as a recovery method, particularly in well-trained individuals. Similarly to the aforementioned studies, the exercise protocol implemented in the current study may not have inflicted enough fatigue to achieve this as some of the performance measures showed little change between pre and post-exercise. This may potentially reduce the magnitude of performance recovery that can actually be achieved through the use of recovery strategies. Future studies should ensure that well-trained participants are put under adequate levels of physical and physiological strain to allow for the need to use various recovery strategies to expedite the recovery process. However, the aim in the current study was to simulate a 'real-world' situation and match similar physical demands that athletes would face during a wheelchair basketball or rugby tournament.

The results of the current study support the finding of Overmayer and Driller (2018) regarding BLa clearance, which revealed no significant difference following ISPC compared to the CON trial. This however contradicts Martin et al (2015) who found a significant improvement in BLa clearance after ISPC treatment. These contrasting results may highlight the physiological differences between able bodied and disabled athletes, particularly those who have sustained a SCI. It also calls attention to an important limitation that needs to be considered when interpreting these results, as each participant's disability can influence the performance measures. The effect a SCI can have on lactate production is a prime example (Thiesen, 2012). Individuals with a SCI have reduced muscle function, which may cause lactate levels to remain low during intense exercise (Leicht, Bishop \& 
Goosey-Tolfrey, 2012). The extent of this will depend on the site of the injury and subsequent level of paralysis. Those with paraplegia will typically be able to reach higher BLa levels than a quadriplegic, simply because they have more active muscle mass in which anaerobic metabolism (and lactate production) can occur (Leicht et al., 2012). Blood flow is also compromised as those with a SCI have a lower stroke volume than their able bodied peers, which can subsequently limit cardiac output and the delivery of oxygenated blood to the working muscles and the removal of lactate (Thiesen, 2012). This may help to explain the ineffectiveness of ISPC on lactate clearance, along with the variation in lactate readings obtained, as there was a mixture of paraplegic and quadriplegic participants included in the study.

Sands et al (2015) revealed an improvement in PPT following ISPC treatment. This differs from the current study in which PPT was not significantly different between ISPC and CON. A major difference between these two studies was the variation in sports represented by the participants recruited by Sands et al (2015), which included weightlifting, gymnastics, triathlon, wrestling and shooting. The exercise session completed by the participants prior to recovery consisted of training for their own sport, and therefore, was not controlled for the type of training, or the intensity and duration, resulting in varying levels of fatigue among the participants. Sands et al (2015) also used a peristaltic compression device, rather than the sequential compression device, used in the current study. Unlike a sequential compression device, in which compartments within the sleeves inflate in a sequential manner and deflate simultaneously, a peristaltic compression device inflates and deflates separate compartments individually (Sands et al., 2015). This would alter the pattern of pressure exerted on the muscles and could possibly influence subsequent PPT measures.

As previously mentioned, the results of this study indicate that ISPC may benefit perceptual measures of recovery immediately after ISPC treatment and up to 24 hours post-exercise. This does not replicate the results of Overmayer and Driller (2018) and Northey et al (2016), who both reported that ISPC made no significant difference to perceptual measures of recovery compared to $\mathrm{CON}$. This may have been caused by contrasting exercise modes. While the current study implemented an interval training format, Overmayer and Driller (2018) included a single maximal effort and Northey et al (2016) incorporated resistance training. The potential variability in ensuing fatigue could influence subsequent perceptual measures of recovery. Similarly to Sands et al (2015), Overmayer and Driller (2018) and 
Northey et al (2016) used lower body exercise to induce fatigue (cycling, back squat), unlike the current study which focused on upper body. During upper body exercise less muscle mass is activated, therefore to work at the same intensity, further effort is required (Schoenmakers, Reed, Van Der Woude \& Hettinga, 2016). There is also some evidence that during exercise the upper body musculature extracts less oxygen than the lower body musculature, therefore decreasing the amount of available oxygen. Increased blood flow is needed to deliver additional oxygen to the working muscles, to compensate for the lower oxygen availability. This will increase the strain on the cardiovascular system and potentially increase feelings of fatigue more so than lower body exercise (Calbert, Holmberg, Rosdahl, van Hall, Jensen-Urstad \& Saltin, 2005).

It is possible the responses to ISPC treatment between athletic populations and individuals with impaired circulation could differ substantially (Sheldon, Roseguini, Thyfault, Crist, Laughlin \& Newcomer, 2012). As previously mentioned, restricted blood redistribution capacity is a common issue for individuals with a SCI, the severity of which is influenced by the height of the injury. Vascular atrophy and reduced vasoconstriction in paralysed limbs inhibits blood flow and venous return at rest and during exercise (Thiesen, 2012). It has been established that ISPC is a promising tool for the treatment of conditions characterised by reduced blood flow (Sheldon et al., 2012). Based on this it could be argued that the participants in the current study with a SCI should benefit from ISPC due to impaired vascular function. However, this did not occur as no difference was found between ISPC and CON. This could be attributed to the superior fitness of the participants compared to the general population, potentially making them more comparable to the able bodied population. This uncertainty highlights the need for more research in this area.

The influence of the placebo effect cannot be discounted, especially with the inclusion of a novel technique such as ISPC. Due to the study design participants could not be blinded to the intervention, making the placebo effect and potential bias impossible to eliminate completely (Halson \& Martin, 2013). In future studies comparing ISPC with another recovery method, other than passive recovery, may be a way to reduce this effect.

All studies discussed, including the current study, followed similar protocols during ISPC treatment. Participants wore the sleeves for similar periods of time. The inflation/deflation cycles were also set to comparable tempos and pressures. Twenty to thirty minutes, and a maximum pressure of $80 \mathrm{mmHg}$ appears to be the standard protocol for most studies. 
However, Zaleska et al (2013) found longer compression times to be more effective in mobilising tissue fluid. They concluded that inflation and deflation times of 50 seconds respectively, allowed for adequate pressure to be achieved. Therefore, extending the inflation time (and pressure exerted) could be a method to increase the effectiveness, particularly in terms of blood flow.

Based on the results of this study upper body ISPC does not appear to influence the physiological processes of recovery for wheelchair team sport athletes following high intensity exercise. However, there is promising evidence that ISPC may positively influence an athlete's perceived feelings of recovery. More research is needed, particularly within the disability sport community, to determine whether this finding warrants ISPC use. 
CHAPTER THREE - Summary, Practical Applications,

Limitations and Future Research 


\section{Summary}

The first chapter of this thesis comprised of a literature review, which examined the current research surrounding the most frequently used recovery methods within the athletic population. These methods included hydrotherapy, active recovery, massage, stretching, napping, nutrition, compression garments, vascular occlusion and ISPC. Training and competition places a high demand on the body, often resulting in skeletal muscle damage, accumulation of metabolic by-products, decreased substrates (fuel stores) and the disruption of hormones (Kenney et al., 2012). In order for athletic recovery to be effective, it needs to assist the body returning to homeostasis, specifically repairing the damage inflicted and returning to pre-exercise levels in preparation for subsequent performance (Luttrell \& Halliwill, 2015). Research focusing on each individual method is able to provide evidence supporting their efficacy in enhancing this process, along with evidence refuting these findings. Many of these recovery methods only seem to be effective in providing relief for some of the consequences of intense training while simultaneously being ineffective or detrimental to other parts of this process. A single recovery method may not be enough to repair skeletal muscle damage, reduce metabolic by-product accumulation and restore substrates, in order for the return to homeostasis to occur following training. Stretching acts as a classic example as it can relieve pain associated with muscle damage but may reduce maximal force and power production, therefore potentially limiting subsequent performance (Cramer et al., 2004; Winchester et al., 2005). A similar effect may occur after hydrotherapy, especially CWI, if the recovery period is limited (e.g. less than 60 minutes). The cold water may assist in thermoregulation for endurance athletes training and/or competing in a hot environment but could also reduce subsequent sprint performance in a team sport athlete (Halson, 2011). Consequently, the selection of recovery methods should be based on a collection of influencing factors: the type of sport (strength, power or endurance), the time in between sessions/events, intensity and duration. Finally, if required, multiple recovery methods or strategies that target different areas of physiological or psychological recovery may be needed to facilitate complete athletic recovery.

The review in Chapter 1 also investigated the current research on recovery in disability sport, which is severely limited compared to research in able bodied athletes. As a result, many of the theories developed through this research are directly applied to athletes with disabilities. This is not always appropriate, especially when the limitations associated with 
many physical disabilities are considered. Therefore the aim of the original study was to provide additional knowledge by investigating the effect of upper body ISPC on the recovery of wheelchair basketball and rugby players after an exercise session, as described in Chapter 2. The main finding suggests ISPC provides marginal benefits in improving subsequent performance, blood lactate and pressure to pain threshold immediately and 24 hours after a high intensity exercise session. Although not statistically significant $(\mathrm{p}>0.05)$, there was a small increase in maximal medicine ball throw following the use of ISPC, when compared to passive recovery. A secondary finding supports the use of ISPC for improving perceived recovery, specifically muscle soreness and fatigue, immediately and 24 hours after a high intensity exercise session. Decreased feelings of muscle soreness and fatigue may improve an athlete's readiness to train, as well as enable athletes to maintain performance during multi-day tournaments. Given the shortage of studies focusing on exercise recovery in disability sport contexts, and the fact that the study in Chapter 2 was completed with a relatively small sample size, further research is necessary. Not only would this provide those involved in disability sport with additional knowledge, it may also elucidate the full capabilities of ISPC as an athlete recovery tool.

\section{Practical Applications}

In terms of subsequent performance and physiological measures, the study in Chapter 2 provides minimal evidence in support of upper body ISPC as a performance recovery tool in wheelchair athletes. However, as perceived muscle soreness and fatigue improved, ISPC may provide some psychological benefits following a high-intensity exercise session. For athletes performing unaccustomed exercise, or returning to training after a break, muscle soreness and fatigue will likely occur, therefore ISPC may provide some relief. Likewise, ISPC could be a useful tool during off season training, when training load is typically high and performance during competitive events is not the primary focus. Athletes might be more likely to train regularly (e.g. daily) if they are not suffering from high levels of muscle soreness or fatigue. Therefore, if athletes avoid training because of this, ISPC may help to prevent soreness and fatigue, leading to better maintenance of training loads. Despite this, there is doubt that this justifies the high cost attached to this piece of equipment, especially when cheaper, alternative recovery methods exist that may provide a similar effect (eg. stretching). 


\section{Limitations}

- A primary limitation of this original study was the small sample size; however this was expected. There are only a couple of local teams in the location where the study took place, resulting in the pool from which the participants were recruited being relatively small. This was further limited as participation was only open to those 18 years and over.

- The use of blood lactate as a physiological measure may have been a limitation, particularly with the inclusion of participants with a SCI. As previously discussed, a SCI compromises blood flow therefore reducing the ability of participants with a SCI to produce high levels of lactate regardless of the intensity at which they are working. Furthermore, blood lactate can be a somewhat crude measure to describe the physiological mechanisms behind certain recovery strategies. The direct measurement of blood flow would have been more appropriate, using plethysmography or ultra-sound techniques, however, the equipment required was not available.

- Despite there being many other potential blood measures (eg. creatine kinase, creactive protein), blood lactate was the only blood measure included in this study. Therefore the results only provided an indication of the effect ISPC had on blood lactate clearance. Including measures such as creatine kinase or c-reactive protein may have provided some insight into how ISPC could influence inflammation associated with exercise.

- Conducting this study without a placebo group makes it impossible to consider the effect the placebo effect may have had on the results, especially when the results suggest ISPC had a positive influence on perceived measures of recovery. We acknowledge this as a limitation and provide ideas for how this might be addressed in future research below.

- Only a CON group was included in this study, meaning ISPC was not compared to any other recovery methods. Given such a limited amount of research has investigated recovery strategies in athletes with disabilities, it is relatively unknown as to how any recovery technique would perform in such a study. It would be interesting to compare ISPC to other commonly used strategies. 


\section{Future Research}

Related to the limitations identified above, there are several lines of inquiry future research could implement.

- Further recovery research involving wheelchair athletes, and athletes with different disabilities is vital for the continued development of high-performance disability sport. Ideally, research should acknowledge the extensive range of physical disabilities represented in disability sport and attempt to conduct more studies involving athletes with a variety of disabilities. This may involve recruiting participants with specific types of disabilities (eg. Cerebral palsy, Muscular Dystrophy, Polio) and investigating whether particular recovery methods are more suitable to meet their individual needs.

- Adding a placebo trial in future research would allow further understanding of the influence athlete belief may have on the efficacy of ISPC. Another possibility is the addition of a brief questionnaire performed pre-study, asking participants of their own beliefs surrounding different recovery techniques. Following the study, these beliefs can be assessed as a covariate in the analysis when evaluating the efficacy of different recovery strategies.

- Comparing ISPC with other recovery methods may indicate the effectiveness of ISPC against other more established recovery methods (eg. hydrotherapy, massage, nutrition strategies).

- Future research may consider incorporating alternative physiological measures which are less likely to be affected by the limitations of various physical disabilities. This is particularly relevant to biochemical measures such as blood lactate, which can be influenced by blood flow. Despite limited knowledge surrounding the influence of an athlete's disability on cortisol, it may act as a useful alternative measure to assess the stress/recovery balance (Colfre-Bolados et al., 2019). As cortisol can be measured using saliva (Cevada, Vasques, Moraes \& Deslandes, 2014), this may reduce the limitation associated with measuring blood flow parameters in athletes with already compromised blood circulation.

- Research focusing on the variety of pressures and treatment durations of ISPC, particularly for wheelchair athletes with varying types of SCI. This may provide more understanding of the appropriateness of ISPC as a recovery tool for athletes with a SCI, especially for those with compromised blood flow. 


\section{References}

Ali Rasooli, S., Koushkie Jahromi, M., Asadmanesh, A., \& Salesi, M. (2012). Influence of massage, active and passive recovery on swimming performance and blood lactate. The Journal of Sports Medicine and Physical Fitness, 52(2), 122-127.

Aragon, A.A., \& Schoenfeld, B.J. (2013). Nutrient timing revisited: Is there a post-exercise anabolic window? Journal of the International Society of Sports Nutrition, 10(1). https://doi.org/10.1186/1550-2783-10-5

Ascensao, A., Leite, M., Rebelo, A.N., Magalhaes, S., \& Magalhaes, J. (2011). Effects of cold water immersion on the recovery of physical performance and muscle damage following a one-off soccer match. Journal of Sport Sciences, 29(3), 217-225. https://doi.org/10.1080/02640414.2010.526132

Asp, S., Daugaard, J.R., Kristiansen, S., Kiens, B., \& Richter, E.A. (1998). Exercise metabolism in human skeletal muscle exposed to prior eccentric exercise. The Journal of Physiology, 509(1), 305-313. https://doi.org/10.1111/j.1469-7793.1998.305bo.x

Baldari, C., Videria, M., Madeira, F., Sergio, J., \& Guidetti, L. (2005). Blood lactate removal during recovery at various intensities below the individual anaerobic threshold in triathletes. Journal of Sports Medicine and Physical Fitness, 45(4), 460-466.

Barnett, A. (2006). Using recovery modalities between training sessions in elite athletes. Does it help? Sports Medicine, 36(9), 781-796. https://doi.org/10.2165/00007256-200636090$\underline{00005}$

Beaven, Cook, Kilduff, Drawer \& Gill (2012). Intermittent lower-limb occlusion enhances recovery after strenuous exercise. Applied Physiology, Nutrition, and Metabolism, 37(6), 1132-1139. https://doi.org/10.1139/h2012-101

Beck, K.L., Thomson, J.S., Swift, R.J., \& von Hurst, P.R. (2015). Role of nutrition in performance enhancement and postexercise recovery. Open Access Journal of Sports Medicine, 6(1), 259-267. https://doi.org/10.2147/OAJSM.S33605

Behm, D.G., \& Chaouachi, A. (2011). A review of the acute effects of static and dynamic stretching on performance. European Journal of Applied Physiology, 111(11), 2633-2651. https://doi.org/10.1007/s00421-011-1879-2.

Bellamy, L.M., Joanisse, S., Grubb, A., Mitchell, C.J., McKay, B.R., Phillips, S.M., Baker, S., \& Parise, G. (2014). The acute satellite cell response and skeletal muscle hypertrophy 
following resistance training. PLoS One, https://doi.org/10.1371/journal.pone.0109739

Bernards, J.R., Sato, K., Haff, G., Bazyler, C.D. (2017). Current research and statistical practices in sport science and a need for change. Sports, 5(4), 87-96.

Bender, T., Nagy, G., Barna, I., Tefner, I., Kadas, E., \& Geher, P. (2007). The effect of physical therapy on beta-endorphin levels. European Journal of Applied Physiology, 100(4), 371382. https://doi.org/10.1007/s00421-007-0469-9

Bessa, A.L., Oliveira, V.N., Agostini, G.G., Oliveira, R.J., Oliveira, A.C., White, G.E., Wells, G.D., Teixeira, D.N., \& Espindola, F.S. (2016). Exercise intensity and recovery: Biomarkers of injury, inflammation, and oxidative stress. Journal of Strength and Conditioning Research, 30(2), 311-309. https://doi.org/10.1519/JSC.0b013e31828f1ee9.

Bhambhani, Y. (2002). Physiology of wheelchair racing in athletes with spinal cord injury. Sports Medicine, 32(1), 23-51.https://doi.org/10.2165/00007256-200232010-00002

Blanchfield, A.W., Lewis-Jones, T.M., Wignall, J.R., Roberts, J.B., \& Oliver, S.J. (2018). The influence of an afternoon nap on the endurance performance of trained runners. European $\begin{array}{lllll}\text { Journal of } & \text { Sport } & \text { Science, } & 18(9), & 1177-1184 .\end{array}$ https://doi.org/10.1080/17461391.2018.147718

Bonnar, D., Bartel, K., Kakoschke, N., \& Lang, C. (2018). Sleep interventions designed to improve athletic performance and recovery: A systematic review of current approaches. Sports Medicine, 48(3), 683-703. https://doi.org/10.1007/s00421-013-2776-7

Borne, R., Hausswirth, C., \& Bieuzen, F. (2016). Relationship between blood flow and performance recovery: A randomized, placebo-controlled study. International Journal of Sports Physiology and Performance, 12(2), 152-160. https://doi.org/10.1123/ijspp.2015$\underline{0779}$

Bourdon, P.C., Cardinale, M., Murray, A., Gastin, P., Kellmann, M., Varley, M.C., Gabbett, T.J., Coutts, A.J., Burgess, D.J., Gregson, W., \& Cable, N.T. (2017). Monitoring athlete training loads: Consensus statement. International Journal of Sports Physiology and Performance, 12(2), 161-170. https://doi.org/10.1123/IJSPP.2017-0208

Brancaccio, P., Maffulli, N., \& Limongelli, F.M. (2007). Creatine kinase monitoring in sport medicine. British Medical Bulletin, 81(1), 209-230.

Broatch, J.R., Petersen, A., \& Bishop, D.J. (2014). Postexercise cold water immersion benefits are not greater than the placebo effect. Medicine \& Science in Sports \& Exercise, 46(11), 21392147. https://doi.org/10.1249/MSS.0000000000000348 
Broderick, V., Uiga, L., \& Driller, M. (2019). Flotation-restricted environmental stimulation therapy improves sleep and performance recovery in athletes. Performance Enhancement and Health, 7(1), 1-9. https://doi.org/10.1016/j.peh.2019.100149

Brophy-Williams, N., Driller, M.W., Halson, S.L., Fell, J.W., \& Kitic, C.M. (2014). Evaluating the Kikuhime pressure monitor for use with sports compression clothing. Sports Engineering, 17(1), 55-60. https://doi.org/10.1007/s12283-013-0125-z

Brown, F., Gissane, C., Howatson, G., van Someren, K., Pedlar, C., \& Hill, J. (2017). Compression garments and recovery from exercise - A meta-analysis. Sports Medicine, 47(11), 22452267. https://doi.org/10.1007/s40279-017-0728-9.

Burke, L.M. (2010). Fuelling strategies to optimize performance: training high or training low? Scandinavian Journal of Medicine and Science in Sports, 20(2), 48-58. https://doi.org/10.1111/j.1600-0838.2010.01185.x

Burke, L.M., Hawley, J.A., Wong, S.H.S., \& Jeukendrup, A.E. (2011). Carbohydrates for training and competition. Journal of Sport Sciences, 29(1), 17-27. https://doi.org/10.1080/02640414.2011.585473

Cafarelli, E., \& Flint, F. (1992). The role of massage in preparation for and recovery from exercise: An overview. Sports Medicine, 14(1), 1-9. https://doi.org/10.2165/00007256-199214010$\underline{00001}$

Calbert, J.A.L., Holmberg, H.C., Rosdahl, H., van Hall, G., Jensen-Urstad, M., \& Saltin, B. (2005). Why do arms extract less oxygen than legs during exercise? American Journal Physiology. Regulative, Integrative and Comparative Physiology, 289(5), 1448-1458.

Caldwell, A.R., Tucker, M.A., Butts, C.L., McDermott, B.P., Vingren, J.L., Kunces, L.J., Lee, E.C., Munoz, C.X., Williamson, K.H., Armstrong, L.E., \& Ganio, M.S. (2017). Effect of caffeine on perceived soreness and functionality following an endurance cycling event. Journal of Strength and Conditioning Research, 31(3), 638-643. https://doi.org/10.1519/JSC.0000000000001608

Camm, A.J., \& Fox, K.A.A. (2018). Strengths and weaknesses of 'real-world' studies involving non-vitamin $\mathrm{K}$ antagonist oral anticoagulants. Open Heart, 5(1). https://doi.org/10.1136/openhrt-2018-000788

Carroll, T.J., Taylor, J.L., \& Gandevia, S.C. (2017). Recovery of central and neuromuscular fatigue after exercise. Journal of Applied Physiology, 122(5), 1068-1076. https://doi.org/10.1152/japplphysiol.00775.2016 
Cevada, T., Vasques, P.E., Moraes, H., \& Deslandes, A. (2014). Salivary cortisol levels in athletes and nonathletes: A systematic review. Hormone and Metabolic Research, 46(13), 905-910. https://doi.org/10.1055/s-0034-1387797

Chen, A.H., Frangos, S.G., Kilaru, S., \& Sumpio, B.E. (2001). Intermittent pneumatic compression devices: Physiological mechanisms of action. European Journal of Vascular and Endovascular Surgery, 21(5), 383-392.https://doi.org/10.1053/ejvs.2001.1348

Chen, C.H., Nosaka, K., Chen, H.L., Lin, M.J., Tseng, K,W., \& Chen, T.C. (2011). Effects of flexibility training on eccentric exercise-induced muscle damage. Medicine and Science in Sports and Exercise, 43(3), 491-500. https://doi.org/10.1249/MSS.0b013e3181f315ad

Claudino, J.G., Cronin, J., Mezencio, B., McMaster, D.T., McGuigan, M., Tricoli, V., Amadio, A.C., Serrao, J.C. (2017). The countermovement jump to monitor neuromuscular status: A meta-analysis. Journal of Science and Medicine in Sport, 20(4), 397-402. https://doi.org/10.1016/j.jsams.2016.08.011

Cockburn, E., Bell, P.G., \& Stevenson, E. (2013). Effect of milk on team sport performance after exercise-induced muscle damage. Medicine and Science in Sports and Exercise, 45(8), 1585-1592. https://doi.org/10.1249/MSS.0b013e31828b7dd0

Cofre-Bolados, C., Requen-Lopez, P., Herrera-Valenzuela, T., Orihuela-Diaz, P., Garcia-Hermoso, A., \& Hackney, A.C. (2019). Testosterone and cortisol responses to HIIT and continuous aerobic exercise in active young me. Sustainability, 11(21), 1-8.

Cohen, J. (1988). Statistical power analysis for the behavioural sciences. (2nd ed,). Lawrence Erlbaum Associates.

Contreras, B. (2012). Figure 1. The Stress-Response Model based on Hans Selye's "General Adaptation Syndrome" [Online image]. The paradox of the strength and conditioning professional. https://bretcontreras.com/the-paradox-of-the-strength-and-conditioning-professional/

Cramer, J.T., Housh, T.J., Johnson, G.O., Miller, J.M., Coburn, J.W., \& Beck, T.W. (2004). Acute effects of static stretching on peak torque in women. Journal of Strength and Conditioning Research, 18(2), 236-241.

Crowe, M.J., O’Connor, D., \& Rudd, D. (2007). Cold water recovery reduces anaerobic performance. International Journal of Sports Medicine, 28(12), 994-998. https://doi.org/10.1055/s-2007-965118

Crowther, F., Sealey, R., Crowe, M., Edwards, A., \& Halson, S. (2017). Team sport athletes' perception and use of recovery strategies: A mixed-methods survey study. BMC Sport Science, Medicine and Rehabilitation, 9(6). https://doi.org/10.1186/s13102-017-0071-3. 
Cunanan, A.J., DeWeese, B.H., Wagle, J.P., Carroll, K.M., Sausaman, R., Hornsby, W.G., Haff, G.G., Triplett, N.T., Pierce, K.C., \& Stone, M.H. (2018). The general adaptation syndrome: A foundation for the concept of periodization. Sports Medicine, 48(4), 787-797. doi: https://doi.org/10.1007/s40279-017-0855-3

Damas, F., Nosaka, K., Libardi, C.A., Chen, T.C., \& Ugrinowitsch, C. (2016). Susceptibility to exercise-induced muscle damage: a cluster analysis with a large sample. International Journal of Sports Medicine, 37(8), 633-640. https://doi.org/10.1055/s-0042-100281

Dattilo, M., Antunes, H.K., Medeiros, A., Monico Neto, M., Souza, H.S., Tufik, S. \& de Mello, M.T. (2011). Sleep and muscle recovery: Endocrinological and molecular basis for a new and promising hypothesis. Medical Hypotheses, 77(2), 220-222.

Davies, D.J., Graham, K.S., \& Chow, C.M. (2010). The effect of prior endurance training on nap sleep patterns. International Journal of Sports Physiology and Performance, 5(1), 87-97. https://doi.org/10.1123/ijspp.5.1.87

Dawson, L.G., Dawson, K.A., \& Tiidus, P.M. (2004). Evaluating the influence of massage on leg strength, swelling, and pain following a half-marathon. Journal of Sports Science \& Medicine, 3(1), 37-43.

De Groot, S., Balvers, I.J.M., Kouwenhoven, S.M., \& Janssen, T.W.J. (2012). Validity and reliability of tests determining performance related components of wheelchair basketball. Journal of Sport Sciences, 30(9), 879-887. https://doi.org/10.1080/02640414.2012.675082

Delextrat, A., Hippocrate, A., Leddington-Wright, S., \& Clarke, N.D. (2014). Including stretches to a massage routine improves recovery from official matches in basketball players. Journal of Strength and Conditioning Research, 28(3), 716-727.

Devlin, J., Paton, B., Poole, L., Sun, W., Ferguson, C., Wilson, J., \& Kemi, O.J. (2014). Blood lactate clearance after maximal exercise depends on active recovery intensity. Journal of Sports Medicine and Physical Fitness, 54(3), 271-278.

Dodd, S., Powers, S.K., Callender, T., \& Brooks, E. (1984). Blood lactate disappearance at various intensities of recovery exercise. Journal of Applied Physiology, Respiratory, Environmental and Exercise Physiology, 57(5), 1462-1465.

Dougherty, K.A., Baker, L.B., Chow, M., \& Kenney, W.L. (2006). Two percent dehydration impairs and six percent carbohydrate drink improves boys basketball skills. Medicine and Science in Sports and Exercise, 38(9),1650-1658. https://doi.org/10.1249/01.mss.0000227640.60736.8e 
Duffield, R., Cannon, J., \& King, M. (2010). The effects of compression garments on recovery of muscle performance following high-intensity sprint and plyometric exercise. Journal of Science and Medicine in Sport, 13(1), 136-140. https://doi.org/10.1016/j.jsams.2008.10.006

Dugas, J.P., Oosthuizen, V., Tucker, R., \& Noakes, T.D. (2006). Drinking “ad libitum” optimises performance and physiological function during $80 \mathrm{~km}$ indoor cycling trials in hot and humid conditions with appropriate convective cooling. Medicine and Science in Sports and Exercise, 38(5). https://doi.org/10.1249/00005768-200605001-00801

Dupuy, O., Douzi, W., Theurot, D., Bosquet, L., \& Dugue, B. (2018). An evidence-based approach for choosing post-exercise recovery techniques to reduce markers of muscle damage, soreness, fatigue, and inflammation: A systematic review with meta-analysis. Frontiers in Physiology, 9. https://doi.org/10.3389/fphys.2018.00403

Edmonds, R., Burkett, B., Leicht, A., \& McKean, M. (2015). Effect of chronic training on heart rate variability, salivary IgA and salivary alpha-amylase in elite swimmers with a disability. PLoS ONE, 10(6), https://doi.org/10.1371/journal.pone.0127749

Evans, G.H., James, L.J., Shirreffs, S.M., \& Maughan, R.J. (2017). Optimizing the restoration and maintenance of fluid balance after exercise-induced dehydration. Journal of Applied Physiology, 122(4), 945-951. https://doi.org/10.1152/japplphysiol.00745.2016

Fagher, K., Jacobsson, J., Timpka, T., Dahlstrom, O., Lexell, J. (2016). The sports-related injuries and illnesses in Paralympic sport study (SRIIPSS): A study protocol for a prospective longitudinal study. BMC Sports Science, Medicine and Rehabilitation, 8(1). https://doi.org/10.1186/s13102-016-0053-x

Fagher, K., \& Lexell, J. (2014). Sports-related injuries in athletes with disabilities. Scandanavian Journal of Medicine and Science in Sports, 24(5), 320-331. https://doi.org/10.1111/sms.12175

Fairchild, T.J., Armstrong, A.A., Rao, A., Liu, H., Lawrence, S., \& Fournier, P.A. (2003). Glycogen synthesis in muscle fibers during active recovery from intense exercise. Medicine and Science in Sports and Exercise, 35(4), 595-602.

Field, T., Hernandez-Reif, M., Diego, M., Schanberg, S., \& Kuhn, C. (2005). Cortisol decreases and serotonin and dopamine increase following massage therapy. The International Journal of Neuroscience, 115(10), 1397-1413. https://doi.org/10.1080/00207450590956459

Franchini, E., Takito, M.Y., Nakamura, F.Y., Matsushigue, K.A., \& Kiss, M.A.P.D.M. (2004). Effects of recovery type after a judo combat on blood lactate removal and performance in an intermittent anaerobic task. Journal of Sports Medicine and Physical Fitness, 43(4), 424431. https://doi.org/10.1007/s00421-009-1134 
Fullagar, H.H.K., Duffield, R., Skorski, S., Coutts, A.J., Julian, R., \& Meyer, T. (2015). Sleep and recovery in team sport: Current sleep-related issues facing professional team-sport athletes. International Journal of Sports Physiology and Performance, 10(8), 950-957. https://.doi.org/10.1123/ijspp.2014-0565

Garcia, C.A., Ribeiro da Mota, G., Scott Leicht, A., \& Marocolo, M. (2017). Ischemic preconditioning and acute recovery of performance in rugby union players. Sports Medicine International Open, 1(3), 107-112. https://doi.org/10.1055/s-0043-111082

Gill, N.D., Beaven, C.M., \& Cook, C. (2006). Effectiveness of post-match recovery strategies in rugby players. British Journal of Sports Medicine, 40(3), 260-263. https://doi.org/10.1136/bjsm.2005.022483

Goosey-Tolfrey, V., Krempien, J., \& Price, M. (2014). Spinal cord injuries. In E. Broad (Ed.), Sports nutrition for Paralympic athletes (pp. 67-90). CRC Press.

Goosey-Tolfrey, V.L., \& Leicht, C.A. (2013). Field-based physiological testing of wheelchair athletes. Sports Medicine, 43(2), 77-91. https://doi.org/10.1007/s40279-012-0009-6

Goosey-Tolfrey, V., \& Price, M. (2010). Physiology of wheelchair sport. In V. Goosey-Tolfrey (Ed.), Wheelchair sport: A complete guide for athletes, coaches and teachers (pp. 47-62). Human Kinetics.

Goosey-Tolfrey, V., Swainson, M., Boyd, C., Atkinson, G., \& Tolfrey, K. (2008). The effectiveness of hand cooling at reducing exercise-induced hyperthermia and improving distance-race performance in wheelchair and able-bodied athletes. Journal of Applied Physiology, 105(1), 37-42. https://doi.org/10.1152/japplphysiol.01084.2007

Goosey-Tolfrey, V., Paulson, T., \& Graham-Paulson, T. (2016). Practical considerations for fluid replacement for athletes with a spinal cord injury. In F. Meyer, Z. Szygula, \& B. Wilk (Eds.), Fluid balance, hydration and athletic performance (pp. 333-355). CRC Press.

Goto, K., \& Morishima, T. (2014). Compression garment promotes muscular strength recovery after resistance exercise. Medicine and Science in Sports and Exercise, 46(12), 2265-2270. https://doi.org/10.1249/MSS.0000000000000359

Grahn, D.A., Cao, V.H., \& Heller, H.C. (2005). Heat extraction through the palm of one hand improves aerobic exercise endurance in a hot environment. Journal of Applied Physiology, 99(3), 972-978. https://doi.org/10.1152/japplphysiol.00093.2005

Greenwood, J.D., Moses, G.E., Bernardino, F.M., Gaesser, G.A., \& Weltman, A. (2008). Intensity of exercise recovery, blood lactate disappearance and subsequent swimming performance. Journal of Sport Sciences, 26(1), 29-34. https://doi.org/10.1080/02640410701287263 
Gunnarsson, T.P., Bendiksen, M., Bischoff, R., Christensen, P.M., Lesivig, B., Madsen, K., Stephens, F., Greenhaff, P., Krustrup, P., \& Bangsbo, J. (2013). Effect of whey protein- and carbohydrate-enriched diet on glycogen resynthesis during the first $48 \mathrm{~h}$ after a soccer game. Scandanavian Journal of Medicine \& Science in Sports, 23(4), 508-515. https://doi.org/10.1111/j.1600-0838.2011.01418.x

Hackney, A.C., \& Lane, A.R. (2015). Exercise and the regulation of endocrine hormones. Progress in Molecular Biology and Translational Science, 135(1), 293-311. https://doi.org/10.1016/bs.pmbts.2015.07.001

Hackney, A.C., \& Walz, E.A. (2013). Hormonal adaptation and the stress of exercise training: the role of glucocorticoids. Trends in Sports Science, 20(4), 165-171.

Halson, S.L. (2011). Does the time frame between exercise influence the effectiveness of hydrotherapy for recovery? International Journal of Sports Physiology and Performance, 6(2), 147-159.

Halson, S.L. (2013). Sleep and the elite athlete. Sports Science Exchange, 26(113), 1-4.

Halson, S.L. (2014), Monitoring training load to understand fatigue in athletes. Sports Medicine, 44(2), 139-147. https://doi.org/10.1007/s40279-014-0253-Z

Halson, S.L. (2015). Recovery techniques for athletes. Aspetar Sports Medicine Journal, 4(6), 1216.

Halson, S.L., \& Martin, D.T. (2013). Lying to win - placebos and sport science. International Journal of Sports Physiology and Performance, 8(6), 597-599. https://doi.org/10.1123/ijspp.8.6.597

Hamlin, M.J. (2007). The effect of contrast temperature water therapy on repeated sprint performance. Journal of Science and Medicine in Sport, 10(6), 398-402. https://doi.org/10.1016/j.jsams.2007.01.002

Hanson, E., Stetter, K., Li, R., \& Thomas, A. (2013). An intermittent pneumatic compression device reduces blood lactate concentrations more effectively than passive recovery after Wingate testing. Journal of Athletic Enhancement, 2(3). https://doi.org/10.4172/2324-9080.1000115

Hemmings, B., Smith, M., Graydon, J., \& Dyson, R. (2000). Effects of massage on physiological restoration, perceived recovery, and repeated sports performance. British Journal of Sports Medicine, 34(2), 109-115.

Herbert, R.D., de Noronha, M., \& Kamper, S.J. (2011). Stretching to prevent or reduce muscle soreness after exercise. The Cochrane Database of Systematic Reviews, 17(4). https://doi.org/10.1002/14651858.CD004577.pub2 
Hilbert, J.E., Sforzo, G.A., \& Swensen, T. (2003). The effects of massage on delayed onset muscle soreness. British Journal of Sports Medicine, $37(1), \quad 75$. http://dx.doi.org/10.1136/bjsm.37.1.72

Hilditch, C.J., Dorrian, J., \& Banks, S. (2017). A review of short naps and sleep inertia: do naps of 30 min or less really avoid sleep inertia and slow-wave sleep? Sleep Medicine, 32(1), 176190. https://doi.org/10.1016/j.sleep.2016.12.016

Hill, J., Howaston, G., van Someren, K., Leeder L., \& Pedlar, C. (2014). Compression garments and recovery from exercise-induced muscle damage: A meta-analysis. British Journal of Sports Medicine, 48(18), 1340-1346. http://doi.org/10.1136/bjsports-2013-092456

Hill, J.A.,Howaston, G., van Someren, K.A., Waishe, I., \& Pedlar, C.R. (2014). Influence of compression garments on recovery after marathon running. Journal of Strength and Conditioning Research, 28(8), 2228-2235.

Hiruma, E., Umimura, M., Naito, H., \& Katamoto, S. (2014). Effects of massage and compression treatment on performance in three consecutive days. Medical Express, 1(6), 328-335. https://doi.org/10.5935/MedicalExpress.2014.06.07

Howatson, G., \& van Someren, K.A. (2008). The prevention and treatment of exercise-induced muscle damage. Sports Medicine, 38(6), 483-503. https://doi.org/10.2165/00007256200838060-00004

Hurley, C.F.,Hatfield, D.L., \& Riebe, D.A. (2013). The effect of caffeine ingestion on delayed onset muscle soreness. Journal of Strength and Conditioning Research, 27(11), 3101-3109. https://doi.org/10.1519/JSC.0b013e3182a99477

Ihsan, M., Watson, G., \& Abbiss, C.R. (2016). What are the physiological mechanisms for postexercise cold water immersion in the recovery from prolonged endurance and intermittent exercise? Sports Medicine, 46(8), 1095-1106. https://doi.org/10.1007/s40279-016-0483-3

Ingram, J., Dawson, B., Goodman, C., Wallman, K., \& Beilby, J. (2009). Effect of water immersion methods on post-exercise recovery from simulated team sport exercise. Journal of Science and Medicine in Sport, 12(3), 417-421. https://doi.org/10.1016/j.jsams.2007.12.011

International Paralympic Committee. (2016). Sport week: History of wheelchair basketball. https://www.paralympic.org/news/sport-week-history-wheelchair-basketball

International Paralympic Committee. (2016). Sport week: History of wheelchair rugby. https://www.paralympic.org/news/sport-week-history-wheelchairrugby?gclid=EAIaIQobChMI_qmc86j15wIVC7aWCh1amwDpEAAYASAAEgILMfD_B $\underline{w E}$ 
International Wheelchair Basketball Federation. (2014). Official player classification manual. https://iwbf.org/wp-content/uploads/2017/09/CLASSIFICATION-MANUAL-2014-2018ENGLISH-FINAL.pdf

International Wheelchair Rugby Federation. (2015). IWRF classification manual. https://www.iwrf.com/resources/iwrf_docs/IWRF_Classification_Manual_3rd_Edition_re v-2015_(English).pdf

Iturricastillo, A., Yanci, J., Granados, C., \& Goosey-Tolfrey, V.L. (2016). Quantifying wheelchair basketball match load: A comparison of heart-rate and perceived-exertion methods. International Journal of Sports Physiology and Performance, 11(4), 508-514. https://doi.org/10.1123/ijspp.2015-0257

Ivy, J.L., Katz, A.L., Cutler, C.L., Sherman, W.M., \& Coyle, E.F. (1988). Muscle glycogen synthesis after exercise: effect of time of carbohydrate ingestion. Journal of Applied Physiology, 64(4), 1480-1485. https://doi.org/10.1152/jappl.1988.64.4.1480

Jakeman, J.R., Byrne, C., \& Eston, R.G. (2010). Lower limb compression garment improves recovery from exercise-induced muscle damage in young, active females. European Journal of Applied Physiology, 109(6), 1137-1144. https://doi.org/10.1007/s00421-010$\underline{1464-0}$

Jensen, J., Rustad, P.I., Kolnes, A.J., \& Lai, Y. (2011). The role of skeletal muscle glycogen breakdown for regulation of insulin sensitivity by exercise. Frontiers in Physiology, 2(112). https://doi.org/10.3389/fphys.2011.00112

Jentjens, R., \& Jeukendrup, A.E. (2003). Determinants of post-exercise glycogen synthesis during short-term recovery. Sports Medicine, 33(2), 117-144.

Keck, N.A., Cuddy, J.S., Hailes, W.S., Dumke, C.L., \& Ruby, B.C. (2015). Effects of commercially available pneumatic compression on muscle glycogen recovery after exercise. Journal of Strength and Conditioning Research, 29(2), 379-385.

Kellmann, M. (2002). Underrecovery and overtraining: Different concepts - similar impact? In M. Kellmann (Ed), Enhancing recovery: Preventing underperformance in athletes (pp. 3-24). Human Kinetics.

Kellman, M. (2010). Preventing overtraining in athletes in high-intensity sports and stress/recovery monitoring. Scandinavian Journal of Medicine \& Science in Sports, 20(2), 95-102. https://doi.org/10.1111/j.1600-0838.2010.01192.x

Kellmann, M., Bertollo, M., Bosquet, L., Brink, M., Coutts, A.J., Duffield, R., Erlacher, D., Halson, S.L., Hecksteden, A., Heidari, J., Kallus, K.W., Meeusen, R., Mujika, I., Robazza, C., Skorski, S., Venter, R., \& Beckmann, J. (2018). Recovery and performance in sport: 
Consensus statement. International Journal of Sports Physiology and Performance, 13(2), 240-245. https://doi.org/10.1123/ijspp.2017-0759

Kellmann M, Kallus KW. (2001). The Recovery-Stress Questionnaire for Athletes: User manual. Human Kinetics.

Kennedy, A.B., Patil, N., \& Trilk, J.L. (2018). 'Recover quicker, train harder, and increase flexibility': massage therapy for elite paracyclists, a mixed-methods study. BMJ Open Sport \& Exercise Medicine, 4(1), 1-7. https://doi.org/10.1136/bmjsem-2017-000319

Kenney, W.L., Wilmore, J.H., \& Costil, D.L. (2012). Physiology of sport and exercise (5th ed.). Human Kinetics.

Kimball, S.R., \& Jefferson, L.S. (2004). Regulation of global and specific mRNA translation by oral administration of branched-chain amino acids. Biochemical and Biophysical Research Communications, 313(2), 423-427. https://doi.org/10.1016/j.bbrc.2003.07.014

Kinugsa, T., \& Kilding, A.E. (2009). A comparison of post-match recovery strategies in youth soccer players. The Journal of Strength and Conditioning Research, 23(5), 1402-1407. https://doi.org/10.1519/JSC.0b013e3181a0226a

Kock, A.J., Pereira, R., \& Machado, M. (2014). The creatine kinase response to resistance exercise. Journal of Musculoskeletal and Neuronal Interactions, 14(1), 68-77.

Kovacs, M.S. (2006). Carbohydrate intake and tennis: are there benefits? British Journal of Sports Medicine, 40(5). https://doi.org/10.1136/bjsm.2005.023291

Kraemer, W.J., Flanagan, S.D., Comstock, B.A., Fragala, M.S., Earp, J.E., Dunn-Lewis, C., Ho, J., Thomas, G.A., Solomon-Hill, G., Penwell, Z.R., Powell, M.D., Wolf, M.R., Volek, J.S., Denegar, C.R., \& Maresh, C.M. (2010). Effects of a whole body compression garment on markers of recovery after a heavy resistance workout in men and women. Journal of Strength and Conditioning Research, 24(3), 804-814.

Kreher, J.B., \& Schwart, J.B. (2012). Overtraining syndrome: A practical guide. Sports Health, 4(2), 128-138. https://doi.org/10.1177/1941738111434406

Kruse, N.T., \& Scheuermann, B.W. (2016). Effect of self-administered on NIRS-measured oxygenation dynamics. Clinical Physiology and Functional Imaging, 36(2), 126-133. https://doi.org/10.1111/cpf.12205

Kruse, N.T., Silette, C.R., \& Scheuermann, B.W. (2016). Influence of passive stretch on muscle blood flow, oxygenation and central cardiovascular responses in healthy young males. American Journal of Physiology, 310(9), 1210-1221. https://doi.org/10.1152/ajpheart.00732.2015 
Krustrup, P., Ortenblad, N., Nielsen, J., Nybo, L., Gunnarsson, T.P., Iaia, F.M., Madsen, K., Stephens, F., Greenhaff, P., \& Bangsbo, J. (2011). Maximal voluntary contraction force, SR function and glycogen resynthesis during the first $72 \mathrm{~h}$ after a high-level competitive soccer game. European Journal of Applied Physiology, 111(12), 2987-2995. https://doi.org/10.1007/s00421-011-1919-y.

Kumar, V., Atherton, P., Smith, K., \& Rennie, M.J. (2009). Human muscle protein synthesis and breakdown during and after exercise. Journal of Applied Physiology, 106(6), 2026-2039. https://doi.org/10.1152/japplphysiol.91481.2008

Laurent, C.M., Green, J.M., Bishop, P.A., Sjokvist, J., Schumacker, R.E., Richardson, M.T.,, \& Curtner-Smith, M. (2011). A practical approach to monitoring recovery: development of a perceived recovery status scale. Journal of Strength and Conditioning Research, 25(3), 620628. https://doi.org/10.1519/JSC.0b013e3181c69ec6

Lee, J.K.W., \& Shirreffs, S.M. (2007). The influence of drink temperature on thermoregulatory responses during prolonged exercise in a moderate environment. Journal of Sport Sciences, 25(9), 975-985. https://doi.org/10.1080/02640410600959947

Leicht, C.A., Bishop, N.C., \& Goosey-Tolfrey, V.L. (2012). Submaximal exercise responses in tetraplegic, paraplegic and non-spinal cord injured elite wheelchair athletes. Scandinavian Journal of Medicine and Science in Sports, 22(6), 729-736. https://doi.org/10.1111/j.1600$\underline{0838.2011 .01328 . \mathrm{x}}$

Leicht, C.A., Bishop, N.C., Paulson, T.A., Griggs, K.E., \& Goosey-Tolfrey, V.L. (2012). Salivary immunoglobulin A and upper respiratory symptoms during 5 months of training in elite tetraplegic athletes. International Journal of Sports Physiology and Performance, 7(3), 210-217.

Levenhagen, D.K., Carr, C., Carlson, M.G., Maron, D.J., Borel, M.J., \& Flakoll, P.J. (2002). Postexercise protein intake enhances whole-body and leg protein accretion in humans. Medicine and Science in Sports and Exercise, 34(5), 828-837. https://doi.org/10.1097/00005768-2002050000-00016

Levenhagen, D.K., Gresham, J.D., Carlson, M.G., Maron, D.J., Borel, M.J., \& Flakoll, P.J. (2001). Postexercise nutrient intake timing in humans is critical to recovery of leg glucose and protein homeostasis. American Journal of Physiology, Endocrinology and Metabolism, 280(6), 982-993. https://doi.org/10.1152/ajpendo.2001.280.6.E982

Levine, J..D., Gordon, N.C., \& Fields, H.L. (1978). The mechanism of placebo analgesia. Lancet, 2(8091), 654-657. https://doi.org/10.1016/s0140-6736(78)92762-9 
Lim, H., Kim, J. W., Lee, K., Seo, D., \& Ko, S. (2018). Hemodynamic effects of different types of pneumatic compression of the lower extremities during anaesthesia induction: A prospective randomized controlled trial. Korean Journal of Anesthesiology, 71(5), 386393. https://doi.org/10.4097/kja.d.18.27179

Loenneke, J.P., Allen, K.M., Mouser, J.G., Thiebaud, R.S., Kim, D., Abe, T., \& Bemben, M.G. (2015). Blood flow restriction in the upper and lower limbs is predicted by limb circumference and systolic blood pressure. European Journal of Applied Physiology, 115(2), 397-405. https://doi.org/10.1007/s00421-014-3030-7

Loenneke, J.P., Thiebaud, R.S., Fahs, C.A., Rossow, L.M., Abe, T., \& Bemben, M.G. (2013). Effect of cuff type on arterial occlusion. Clinical Physiology and Functional Imaging, 33(4), 325-327. https://doi.org/10.1111/cpf.12035

Lund, H., Vestergaard-Poulsen, P., Kanstrup, I.L., \& Sejrsen, P. (1998). The effect of passive stretching on delayed onset muscle soreness, and other detrimental effects following eccentric exercise. Scandanavian Journal of Medicine \& Science in Sports, 8(4), 216-221. https://doi.org/10.1111/j.1600-0838.1998.tb00195.x

Luttrell, M.J., \& Halliwill, J.R. (2015). Recovery from exercise: Vulnerable state, window of opportunity or crystal ball? Frontiers in Physiology, 6(204), 1-6. https://doi.org/10.3389/fphys.2015.00204

MacIntyre, D.L., Reid, W.D., Lyster, D.M., \& McKenzie, D.C. (2000). Different effects of strenuous eccentric exercise on the accumulation of neutrophils in muscle in women and men. European Journal of Applied Physiology, 81(1), 47-53. https://doi.org/10.1007/PL00013796

Mah, C.D., Mah, K.E., Kezirian, E.J., \& Dement, W.C. (2011). The effects of sleep extension on the athletic performance of collegiate basketball players. Sleep, 34(7), 943-950. https://doi.org/10.5665/SLEEP.1132

Malone, L.A., Morgulec-Adamowicz, N., \& Orr, K. (2011). Contribution of sport science to performance - wheelchair rugby. In Y.C. Vanlandewijck \& W.R. Thompson(Eds.), The paralympic athlete (pp. 51-73). Wiley-Blackwell.

Mancinelli, C.A., Davis, D.S., Aboulhosn, L., Brady, M., Eisenhofer, J., \& Foutty, S. (2006). The effects of massage on delayed onset muscle soreness and physical performance in female collegiate athletes. Physical Therapy in Sport, 7(1), 5-13. https://doi.org/10.1016/j.ptsp.2005.10.004 
Marcello, R.T., Fortini, L., \& Greer, B.K. (2019). Intermittent pneumatic compression boot use elevates blood lactate during subsequent exercise. International Journal of Exercise Science, 12(2), 385-392.

Maridakis, V., O’Connor, P.J., Dudley, G.A., \& McCully, K.K. (2007). Caffeine attenuates delayed-onset muscle pain and force loss following eccentric exercise. The Journal of Pain, 8(3), 237-243. https://doi.org/10.1016/j.jpain.2006.08.006

Martin, J.S., Friedenreich, Z.D., Borges, A.R., \& Roberts, M.D. (2015). Acute effects of peristaltic pneumatic compression on repeated anaerobic exercise performance and blood lactate clearance. Journal of Strength and Conditioning Research, 29(10), 2900-2906.

McCully, K.K. (2010). The influence of passive stretch on muscle oxygen saturation. Advances in Experimental Medicine and Biology, 662(1), 317-322. https://doi.org/10.1007/978-1-4419$\underline{1241-1 \_45}$

McGrath, R.P., Whitehead, J.R., \& Caine, D.J. (2014). The effects of proprioceptive neuromuscular facilitation stretching on post-exercise delayed onset muscle soreness in young adults. International Journal of Exercise Science, 7(1), 14-21.

McGuigan, M.R. (2007). Monitoring training and performance in athletes. Human Kinetics.

McLellan, C.P., Lovell, D.I. \& Gass, G.C. (2010). Creatine kinase and endocrine responses of elite players pre, during, and post rugby league match play. Journal of Strength and Conditioning Research, 24(11), 2908-2919.

Menzies, P., Menzies, C., McIntyre, L., Paterson, P., Wilson, J., \& Kemi, O.J. (2010). Blood lactate clearance during active recovery after an intense running bout depends on the intensity of the active recovery. Journal of Sport Sciences, 28(9), 975-982. https://doi.org/10.1080/02640414.2010.481721

Merson, S.J., Maughan, R.J., \& Shirreffs, S.M. (2008). Rehydration with drinks differing in sodium concentration and recovery from moderate exercise-induced hypohydration in man. European Journal of Applied Physiology, 103(5), 585-594. https://doi.org/10.1007/s00421$\underline{008-0748-0}$

Mika, A., Oleksy, L., Kielnar, R., Wodka-Natkaniec, E., Twardowska, M., Kaminski, K., \& Malek, Z. (2016). Comparison of two different modes of active recovery on muscles performance after fatiguing exercise in mountain canoeist and football players. Plos One, 11(10). https://doi.org/10.1371/journal.pone.0164216

Mizuno, S., Arai, M., Todoko, F., Yamada, E., \& Goto, K. (2017). Wearing lower-body compression garment with medium pressure impaired exercise-induced performance 
decrement during prolonged running. PLOS ONE, 12(5). https://doi.org/10.1371/journal.pone.0178620

Montgomery, P., \& Hopkins, W. (2013). The effects of game and training loads on perceptual responses of muscle soreness in Australian football. International Journal of Sports Physiology and Performance, 8(3), 312-318.

Montgomery, P.G., Pyne, D.B., Hopkins, W.G., Dorman, J.C., Cook, K.C., \& Minahan, C.L. (2008). The effect of recovery strategies on physical performance and cumulative fatigue in competitive basketball. Journal of Sport Sciences, 26(11), 11351145. https://doi.org/10.1080/02640410802104912

Mujika, I., \& Burke, L.M. (2010). Nutrition in team sports. Annals of Nutrition \& Metabolism, 57(2). https://doi.org/10.1159/000322700

Murray, A., Fullagar, H., Turner, A., \& Sproule, J. (2018). Recovery practices in division 1 collegiate athletes in North America. Physical Therapy in Sport, 32(1), 67-73. https://doi.org/10.1016/j.ptsp.2018.05.004

Nedelec, M., McCall, A., Carling, C., Legall, F., Berthoin, S., \& Dupont, G. (2013). Recovery in soccer: Part II - recovery strategies. Sports Medicine, 43(1), 9-22. https://doi.org/10.1007/s40279-012-0002-0.

Neric, F.B., Beam, W.C., Brown, L.E., \& Wiersma, L.D. (2009). Comparison of swim recovery and muscle stimulation on lactate removal after sprint swimming. The Journal of Strength and Conditioning Research, 23(9), 2560-2567.

Noakes, T.D. (2007). The central governor model of exercise regulation applied to the marathon. Sports Medicine, 37(4), 374-377.

Northey, J.M., Rattray, B., Argus, C.K., Etxebarria, N., \& Driller, M.W. (2016). Vascular occlusion and sequential compression for recovery after resistance exercise. Journal of Strength and Conditioning Research, 30(2), 533-539.

Nosaka, K., Newton, M., \& Sacco, P. (2002). Delayed-onset muscle soreness does not reflect the magnitude of eccentric exercise-induced muscle damage. Scandanavian Journal of Medicine \& Science in Sports, 12(6), 337-346. https://doi.org/10.1034/j.1600$\underline{0838.2002 .10178 . x}$

Obal, F., \& Krueger, J.M. (2004). GHRH and sleep. Sleep Medicine Reviews, 8(5), 367377. https://doi.org/10.1016/j.smrv.2004.03.005.

O’Donnell, S., Beaven, C.M., \& Driller, M. (2018). The influence of match-day napping in elite female netball athletes. International Journal of Sports Physiology and Performance, 13(9), 1143-1148. https://doi.org/10.1123/ijspp.2017-0793 
Ogai, R., Yamane, M., Matsumoto, T., \& Kosaka, M. (2008). Effects of petrissage massage on fatigue and exercise performance following intensive cycle pedalling. British Journal of Sports Medicine, 42(10), 834-838. https://doi.org/10.1136.bjsm.2007.044396

Otsuki, A., Fujita, E., Ikegawa, S., \& Kuno-Mizumura, M. (2011). Muscle oxygenation and fascicle length during passive muscle stretching in ballet-trained subjects. International Journal of Sports Medicine, 32(7), 496-502. https://doi.org/10.1055/s-0031-1275297

Overmayer, R.G., \& Driller, M.W. (2015). Pneumatic compression fails to improve performance recovery in trained cyclists. International Journal of Sports Physiology and Performance, 13(4), 490-495. https://doi.org/10.1123/ijspp.2017-0207

Ozmen, T., Yuktasir, B., Un Yildirim, N., Yalcin, B., \& Willems, M.E.T. (2014). Explosive strength training improves speed and agility in wheelchair basketball athletes. Revista Brasileira de Medicina do Esporte, 20(2), 97-100. https://dx.doi.org/10.1590/1517$\underline{86922014200201568}$

Paccotti, P., Minetto, M., Terzolo, M., Ventura, M., Ganzit, G.P., Borrione, P., Termine, A., \& Angeli, A. (2005). Effects of high-intensity isokinetic exercise on salivary cortisol in athletes with different training schedules: Relationships to serum cortisol and lactate. International Journal of Sports Medicine, 26(9), 747-755. https://doi.org/10.1055/s-2004$\underline{830449}$

Page, P. (2012). Current concepts in muscle stretching for exercise and rehabilitation. International Journal of Sports Physical Therapy, 7(1), 109-119.

Page, W., Swan, R., \& Patterson, S.D. (2017). The effect of intermittent lower limb occlusion on recovery following exercise-induced muscle damage: A randomized control trial. Journal of Science and Medicine in Sport, 20(8), 729-733. https://doi.org/10.1016/j.jsams.2016.11.015

Park, G., Kim, C.W., Park, S.B., Kim, M.J., \& Jang, S.H. (2011). Reliability and usefulness of the pressure pain threshold measurement in patients with myofascial pain. Annals of Rehabilitation Medicine, 35(3), 412-417. https://doi.org/10.5535/arm.2011.35.3.412

Parkin, J.A., Carey, M.F., Martin, I.K., Stojanovska, L., \& Febbraio, M.A. (1997). Muscle glycogen storage following prolonged exercise: effect of timing of ingestion of high glycemic index food. Medicine and Science in Sports and Exercise, 29(2), 220-224. https://doi.org/10.1097/00005768-1997020000-00009

Paulson, T.A.W., \& Goosey-Tolfrey, V.L. (2017). Current perspectives on profiling and enhancing wheelchair court-sport performance. International Journal of Sports Physiology and Performance, 12(3), 275-286. https://doi.org/10.1123/ijspp.2016-0231 
Paulson, T.A.W., Mason, B., Rhodes, J., \& Goosey-Tolfrey, V.L. (2015). Individualized internal and external training load relationships in elite wheelchair rugby players. Frontiers in Physiology, 6. https://doi.org/10.3389/fphys.2015.00388

Peake, J.M. (2019). Recovery after exercise: What is the current state of play? Current Opinion in Physiology, 10(1), 17-26. https://doi.org/10.1016/j.cophys.2019.03.007

Peiffer, J.J., Abbiss, C.R., Nosaka, K., Peake, C.M., \& Laursen, P.B. (2009). Effect of cold water immersion after exercise in the heat on muscle function, body temperatures, and vessel diameter. Journal of Science and Medicine in Sport, 12(1), 91-96. https://doi.org/10.10.16/j.jsams.2007.10.011

Penailillo, L., Blazevich, A., Numazawa, H., \& Nosaka, K. (2015). Rate of force development as a measure of muscle damage. Scandinavian Journal of Medicine and Science in Sports, 25(3), 417-427. https://doi.org/10.1111.sms.12241

Perez-Idarraga, A., \& Aragon-Vargas, L.F. (2014). Postexercise hydration: Potassium-rich drinks versus water and a sports drink. Applied Physiology, Nutrition and Metabolism, 39(10), 1167-1174. https://doi.org/10.1139/apnm-2013-0434

Petit, E., Mougin, F., Bourdin, H., Tio, G., \& Haffen, E. (2014). A 20-min nap in athletes changes subsequent sleep architecture but does not alter physical performances after normal sleep or 5-h phase-advance conditions. European Journal of Applied Physiology, 114(2), 305315. https://doi.org/10.1007/s00421-013-2776-7

Pollock, S., Gaoua, N., Johnston, M.J., Cooke, K., Girard, O., \& Mileva, K.N. (2019). Training regimes and recovery monitoring practices of elite British swimmers. Journal of Sports Science \& Medicine, 18(3), 577-585.

Poppendieck, W., Wegmann, M., Ferrauti, A., Kellmann, M., Pfeiffer, M., \& Meyer, T. (2016). Massage and performance recovery: A meta-analytical review. Sports Medicine, 46(2), 183204. https://doi.org/10.1007/s40279-015-0420-X

Pournot, H., Bieuzen, F., Duffield, R., Leprêtre, P., Cozzolino, C., \& Hausswirth, C. (2011). Short term effects of various water immersions on recovery from exhaustive intermittent exercise. European Journal of Applied Physiology, 111(7), 1287-1295. https://doi.org/10.1007/s00421010-1754-6

Price, M.J., \& Campbell, I.G. (2003). Effects of spinal cord lesion level upon thermoregulation during exercise in the heat. Medicine and Science in Sports and Exercise 35(7), 1100-1107. https://doi.org/10.1249/01.MSS.0000074655.76321.D7 
Pruscino, C.L., Halson, S., \& Hargreaves, M. (2013). Effects of compression garments on recovery following intermittent exercise. European Journal of Applied Physiology, 113(6), 15851596. https://doi.org/10.1007/s00421-012-2576-5.

Prystypa, T., Stefaniak, T., \& Rudenko, R. (2017). Impact of athletic recovery parameters of hemodynamics in disabled powerlifters with cerebral palsy. Pedagogics, Psychology, Medical-biological Problems of Physical Training and Sports, 21(3):131-138. https://doi.org/10.15561/18189172.2017.0306

Reilly, T., \& Edwards, B. (2007). Altered sleep-wake cycles and physical performance in athletes. Physiology \& Behaviour, 90(2), 274-284. https://doi.org/10.1016/j.physbeh.2006.09.017

Rinaud, D., Calmels, P., Roche, F., Mongold, J.J., Trudeau, F., \& Devillard, X. (2007). Effects of graduated compression stockings on cardiovascular and metabolic responses to exercise and exercise recovery in persons with spinal cord injury. Archives of Physical Medicine and Rehabilitation, 88(6), 703-709. https://doi.org/10.1016/j.apmr.2007.03.023

Roberts, L.A., Raastad, T., Markworth, J.F., Figueiredo, V.C., Egner, I.M., Shield, A., CameronSmith, D., Coombes, J.S., \& Peake, J.M. (2015). Post-exercise cold water immersion attenuates acute anabolic signalling and long-term adaptations in muscle to strength training. The Journal of Physiology, 593(18), 4285-4301. https://doi.org/10.1113/JP270570

Robey, E., Dawson, B., Goodman, C., \& Beilby, J. (2009). Effect of post exercise recovery procedures following strenuous stair-climb running. Research in Sports Medicine, 17(4), 245-259. https://doi.org/10.1080/15438620902901276

Rokitzki, L., Logemann, E., Sagredos, A.N., Murphy, M., Wetzel-Roth, W., \& Keul, J. (1994). Lipid peroxidation and antioxidative vitamins under extreme endurance stress. Acta Physiologica Scandanavia, 151(2), 149-158. $\quad$ https://doi.org/10.1111/j.17481716.1994.tb09732.x

Rugg, S., \& Sternlicht, E. (2013). The effect of graduated compression tights, compared with running shorts, on countermovement jump performance before and after submaximal running. Journal of Strength and Conditioning Research, 27(4), 1067-1073.

Russell, M., Sparkes, W., Northeast, J., Cook, C.J., Bracken, R.M., \& Kilduff, L.P. (2016). Relationships between match activities and peak power output and Creatine Kinase responses to professional reserve team soccer match-play. Human Movement Science, 45(1), 96-101. https://doi.org/10.1016/j.humov.2015.11.011 
Sands, W.A., McNeal, J.R., Murray, S.R., Ramsey, M.W., Sato, K., Mizuguchi, S., \& Stone, M.H. (2013). Stretching and its effects on recovery: A review. Strength and Conditioning Journal, 35(5), 30-36. https://doi.org/10.1519/SSC.0000000000000004

Sands, W.A., McNeal, J.R., Murray, S.R., \& Stone, M.H. (2015). Dynamic compression enhances pressure-to-pain threshold in elite athlete recovery. Journal of Strength and Conditioning Research, 29(5), 1263-1272.

Saw, A.E., Main, L.C., \& Gastin, P.B. (2015). Monitoring the athlete training response: subjective self-reported measures trump commonly used objective measures: A systematic review. British Journal of Sports Medicine, 50(5), 281-291.

Sawka, M.N., Burke, L.M., Eichner, E.R., Maughan, R.J., Montain, S.J., \& Stachenfeld, N.S. (2007). American College of Sports Medicine position stand. Exercise and fluid replacement. Medicine and Science in Sports and Exercise, 39(2), 377-390. https://doi.org/10.1249/mss.0b013e31802ca597

Sawka, M.N., \& Noakes, T.D. (2007). Does dehydration impair exercise performance? Medicine and Science in Sports and Exercise, 39(8), 1209-1217. https://doi.org/10.1249/mss.0b013e318124a664

Sawynok, J. (2016). Adenosine receptor targets for pain. Neuroscience, 338(1), 1-18. https://doi.org/10.1016/j.neuroscience.2015.10.031

Schniepp, J., Campbell, T.S., Powell, K.L., \& Pincivero, D.M. (2002). The effects of cold water immersion on power output and heart rate in elite cyclists. Journal of Strength and Conditioning Research, 16(4), 561-566.

Schoenmakers, P., Reed, K., Van Der Woude, L., \& Hettinga, F.J. (2016). High intensity interval training in handcycling: the effects of a 7-week training intervention in able-bodied men. Frontiers in Physiology, 7(638), 561-566. https://doi.org/10.3389/fphys.2016.00638

Sheldon, R.D., Roseguini, B.T., Thyfault, J.P., Crist, B.D., Laughlin, M.H., \& Newcomer, S.C. (2012). Acute impact of intermittent pneumatic leg compression frequency on limb hemodynamics, vascular function, and skeletal muscle gene expression in humans. Journal of Applied $\quad$ Physiology,112(12), 2099-2109. https://doi.org/10.1152/japplphysiol.00042.2012

Shirreffs, S.M., Aragon-Vargas, L.F., Keil, M., Love, T.D., \& Phillips, S. (2007). Rehydration after exercise in the heat: a comparison of 4 commonly used drinks. International Journal of Sport Nutrition and Exercise Metabolism, 17(3), 244-258. https://doi.org/10.1123/ijsnem.17.3.244 
Simic, L., Sarabon, N., \& Markovic, G. (2013). Does pre-exercise static stretching inhibit maximal muscular performance? A meta-analytical review. Scandinavian Journal of Medicine and Science in Sports, 23(2), 131-148. https://doi.org/10.1111/j.1600-0838.2012.01444.x

Singh, T.K., Guelfi, K.J., Landers, G., Dawson, B., \& Bishop, D. (2011). A comparison of muscle damage, soreness and performance following a simulated contact and non-contact team sport activity circuit. Journal of Science and Medicine in Sport, 14(5), 441-446. https://doi.org/10.1016/j.jsams.2011.03.008

Smith, L.L., Keating, M.N., Holbert, D., Spratt, D.J., McCammon, M.R., Smith, S.S., \& Israel, R.G. (1994). The effects of athletic massage on delayed onset muscle soreness, creatine kinase and neutrophil count: A preliminary report. The Journal of Orthopaedic and Sports Physical Therapy, 19(2), 93-99.

Spaccarotella, K.J., \& Walter, W.D. (2011). The effects of low fat chocolate milk on postexercise recovery in collegiate athletes. Journal of Strength and Conditioning Research, 25(12), 3456-3460.

St Clair Gibson, A., \& Noakes, T.D. (2004). Evidence for complex system integration and dynamic neural regulation of skeletal muscle recruitment during exercise in humans. British Journal of Sports Medicine, 38(6), 797-806.

Suzuki, M., Umeda, T., Nakaji, S., Shimoyama, T., Mashiko, T., \& Sugawara, K. (2004). Effect of incorporating low intensity exercise into the recovery period after a rugby match. British Journal of Sports Medicine, 38(4), 436-440. https://doi.org/10.1136/bjsm.2002.004309

Taveres, F., Smith, T.B., \& Driller, M. (2017). Fatigue and recovery in rugby: A review. Sports Medicine, 47(8), 1515-1530. https://doi.org/10.1007/s40279-017-0679-1

Theisen, D. (2012). Cardiovascular determinants of exercise capacity in the Paralympic athlete with spinal cord injury. Experimental Physiology, 97(3), 319-324. https://doi.org/10.1113/expphysiol.2011.063016

Thomas, K., Morris, P., \& Stevenson, E. (2009). Improved endurance capacity following chocolate milk consumption compared with 2 commercially available sport drinks. Applied Physiology, Nutrition and Metabolism, 34(1), 78-82.

Thorpe, R., \& Sunderland, C. (2012). Muscle damage, endocrine, and immune marker response to a soccer match. Journal of Strength and Conditioning Research, 26(10), 2783-2790. https://doi.org/10.1519/JSC.0b013e318241e174

Tipton, K.D., Rasmussen, B.B., Miller, S.L., Wolf, S.E., Owens-Stovall, S.K., Petrini, B.E., \& Wolfe, R.R. (2001). Timing of amino acid-carbohydrate ingestion alters anabolic response 
of muscle to resistance exercise. American Journal of Physiology. Endocrinology and Metabolism, 281(2), 197-206. https://doi.org/10.1152/ajpendo.2001.281.2.E197

Toubekis, A.G., Tsolaki, A., Smilios, I., Douda, H.T., Kourtesis, T., \& Tokmakidis, S.P. (2008). Swimming performance after passive and active recovery of various durations. International Journal of Sports Physiology and Performance, 3(3), 375-386. https://doi.org/10.1123/ijspp.3.3.375

Tweedy, S., \& Diaper, N. (2010). Introduction to wheelchair sport. In V. Goosey-Tolfrey (Ed.), Wheelchair sport: A complete guide for athletes, coaches and teachers (pp. 3-28). Human Kinetics.

Vaile, J., Halson, S., Gill, N., \& Dawson, B. (2008). Effects of cold water immersion on repeat cycling performance and thermoregulation in the heat. Journal of Sport Sciences, 26(5), 431-440. https://doi.org/10.1080/02640410701567425

Vaile, J., Stefanovic, B., \& Askew, C.D. (2016). Effect of lower limb compression on blood flow and performance in elite wheelchair rugby athletes. The Journal of Spinal Cord Medicine, 39(2), 206-211. https://doi.org/10.1179/2045772314Y.0000000287

Venter, R.E. (2014). Perceptions of team athletes on the importance of recovery modalities. $\begin{array}{lllll}\text { European Journal of Sport } & \text { Science, }\end{array}$ https://doi.org/10.1080/17461391.2011.643924

Venter, R.E., Potgieter, J.R., \& Barnard, J.G. (2010). The use of recovery modalities by elite South African team athletes. South African Journal for Research in Sport, Physical Education and Recreation, 32(1), 133-145.

Versey, N., Halson, S., \& Dawson, B. (2011). Effect of contrast water therapy duration on recovery of cycling performance: a dose-response study. European Journal of Applied Physiology, 111(1), 37-46. https://doi.org/10.1007/s00421-010-1614-4

Versey, N.G, Halson, S.L, \& Dawson, B.T. (2012). Effect of contrast water therapy duration on recovery of running performance. International Journal of Sports Physiology and Performance, 7(2), 130-140. https://doi.org/10.1123/ijspp.7.2.130

Walloe, L. (2016). Arterio-venous anastomoses in the human skin and their role in temperature control. Temperature, 3(1), 92-103. https://doi.org/10.1080/23328940.2015.1088502

Warren, G.L., Lowe, D.A., \& Armstrong, R.B. (1999). Measurement tools used in the study of eccentric contraction-induced injury. Sports Medicine, 27(1), 43-59. https://doi.org/10.2165/00007256-199927010-00004

Waterhouse, J., Atkinson, G., Edwards, B., \& Reilly, T. (2007). The role of a short post-lunch nap in improving cognitive, motor, and sprint performance in participants with partial sleep 
deprivation. Journal of Sport Sciences, 25(14), 1557-1566. https://doi.org/10.1080/02640410701244983

Weerapong, P., Hume, P.A., \& Kolt, G.S. (2005). The mechanisms of massage and effects on performance, muscle recovery and injury prevention. Sports Medicine, 35(3), 235-256. https://doi.org/0112--1642/05/0003-0235

Wesensten, N.J., Killgore, W.D.S., \& Balkin, T.J. (2005). Performance and alertness effects of caffeine, dextroamphetamine, and modafinil during sleep deprivation. Journal of Sleep Research, 14(3), 255-266.

Wiewelhove, T., Raeder, C., Meyer, T., Kellmann, M., Pfeiffer, M., \& Ferrauti, A.(2015). Markers for routine assessment of fatigue and recovery in male and female team sport athletes during high-intensity interval training. PLoS ONE10(10). https://doi.org/10.1371/journal.pone.0139801

Williams, C., \& Rollo, I. (2015). Carbohydrate nutrition and team sport performance. Sports Science Exchange, 28(140), 1-7.

Williams, N., Russell, M., Cook, C.J., \& Kilduff, L.P. (2018). The effect of lower limb occlusion on recovery following sprint exercise in academy rugby players. Journal of Science and Medicine in Sport, 21(10), 1095-1099. https://doi.org/10.1016/j.jsams.2018.02.012

Winchester, J.B., Nelson, A.G., Landin, D., Young, M.A., \& Schexnayder, I.C. (2005). Static stretching impairs sprint performance in collegiate track and field athletes. Journal of Strength and Conditioning Research, 22(1), 13-18.

Winke, M., \& Williamson, S. (2018). Comparison of a pneumatic compression device to a compression garment during recovery from DOMS. International Journal of Exercise Science, 11(3), 375-383.

Yamane, M.,Teruya, H., Nakano, M., Ogai, R., Ohnishi, N., \& Kosaka, M. (2006). Post-exercise leg and forearm flexor muscle cooling in humans attenuates endurance and resistance training effects on muscle performance and circulatory adaptation. European Journal of Applied Physiology, 96(5), 572-580. https://doi.org/10.1007/s00421-005-0095-3

Yanci, J., Granados, C., Otero, M., Badiola, A., Olasagasti, J., Bidaurrazaga-Letona, I., Iturricastillo, A., \& Gill, S.M. (2015). Sprint, agility, strength and endurance capacity in wheelchair basketball players. Biology of Sport, 32(1), 71-78. https://doi.org/10.5604/20831862.1127285

Yfanti, C., Fischer, C.P., Nielsen, S., Akerstrom, T., Nielsen, A.R., Veskoukis, A.S., Kouretas, D., Lykkesfeldt, J., Pilegaard, H., \& Pedersen, B.K. (2012). Role of vitamin C and E 
supplementation in IL-6 in response to training. Journal of Applied Physiology, 112(6), 990-1000. https://doi.org/10.1152/japplphysiol.01027.2010

Young, W.B. (2007). The use of static stretching in warm-up for training and competition. International Journal of Sports Physiology and Performance, 2(2), 212-216. https://doi.org/10.1123/ijspp.2.2.212

Zadkhosh, S.M., Ariaee, E., Atri, A.E., Rashidlamir,A., \& Saadatyar, A. (2015). The effect of massage therapy on depression, anxiety and stress in adolescent wrestlers. International Journal of Sport Studies, 5(3), 321-327.

Zaleska, M., Olszewski, W., Jain, P., Gogia, S., Rekha, A., Mishra, S., \& Durlik, M. (2013). Pressures and timing of intermittent pneumatic compression devices for efficient tissue fluid and lymph flow in limbs with lymphedema._Lymphatic Research and Biology, 11(4), 227232. https://doi.org/10.1089/1rb.2013.0016.

Zehnder, M., Muelli, M., Buchli, R., Kuehne, G., \& Boutellier, U. (2004). Further glycogen decrease during early recovery after eccentric exercise despite a high carbohydrate intake. European Journal of Nutrition, 43(3), 148-159. https://doi.org/10.1007/s00394-004-0453$\underline{7}$

Zhao, J.M., He, M.L., Xiao, Z.M., Li, T.S., Wu, H., \& Jiang, H. (2014). Different types of intermittent pneumatic compression devices for preventing venous thromboembolism in patients after total hip replacement. Cochrane Database of Systematic Reviews, 12. https://doi.org/10.1002/14651858.CD009543.pub3

Zoppi, C.C., Hohl, R., Silva, F.C., Lazarim, F.L., Antunes Neto, J.M.F., Stancanneli M., \& Macedo, D.V. (2006). Vitamin C and E supplementation effects in professional soccer players under regular training. Journal of the International Society of Sports Nutrition. 3(2), 37-44. 


\section{Appendices}

\section{Appendix 1 - Participant Consent Form}

\section{Consent Form}

\section{Please tick to indicate you consent to the following}

I have read or have had read to me in my first language, and I understand the Participant Information Sheet.

I have been given enough time to consider whether to participate in this study.

I have had the opportunity to use a legal representative, whanau/ family support or a friend to help me ask questions and understand the study.

I am satisfied with the answers I have been given regarding the study and I have a copy of this consent form and information sheet.

I understand that taking part in this study is voluntary (my choice) and that I may withdraw from the study at any time.

I consent to the research staff collecting and processing my information.

If I decide to withdraw from the study, I agree that the information collected about me up to the point when I withdraw may continue to be processed.

I understand that there may be injury risks associated with my involvement in the study.

I understand that my participation in this study is confidential and that no material, which could identify me personally, will be used in any reports on this study.

I understand the compensation provisions in case of injury during the study.

I know who to contact if I have any questions about the study in general. 
I understand my responsibilities as a study participant.

I wish to receive a summary of the results from the study.

Yes $\square \quad$ No $\square$

\section{Declaration by participant:}

I hereby consent to take part in this study.

Participant's name:

Signature: Date:

\section{Declaration by member of research team:}

I have given a verbal explanation of the research project to the participant and have answered the participant's questions about it.

I believe that the participant understands the study and has given informed consent to participate.

Researcher's name:

Signature:

Date: 
Appendix 2 - Ethics Approval

Health and Disability Ethics

Committees Ministry of Health 133

Molesworth Street PO Box 5013

Wellington 6011

08004 ETHICS hdecs@moh.govt.nz

25 June

2019

Ms Alison Oliver

$7 \quad$ Montrose

Crescent

Huntington

0211721882

Hamilton 3210

Dear Ms

Oliver,

\section{Re: Ethics ref: 19/NTB/84}

Study title: Investigating the use of an intermittent sequential pneumatic compression arm sleeve for recovery following a high intensity wheelchair basketball session

I am pleased to advise that this application has been approved by the Northern B Health and Disability Ethics Committee. This decision was made through the HDEC-Expedited Review pathway.

HDEC approval for this study is subject to the following conditions being met prior to the commencement of the study in New Zealand. It is your responsibility, and that of the study's sponsor, to ensure that these conditions are met. No further review by the Northern B Health and Disability Ethics Committee is required.

\section{Standard}

conditions:

1. Before the study commences at any locality in New Zealand, all relevant

regulatory approvals must be obtained. 
2. Before the study commences at any locality in New Zealand, it must be

registered in a clinical trials registry. This should be a WHO-approved registry (such as the Australia New Zealand Clinical Trials Registry, www.anzctr.org.au) or https://clinicaltrials.gov/.

3. Before the study commences at each given locality in New Zealand, it must be

authorised by that locality in Online Forms. Locality authorisation confirms that the locality is suitable for the safe and effective conduct of the study, and that local research governance issues have been addressed.

Please refer to the Standard Operating Procedures for Health and Disability Ethics Committees (available on www.ethics.health.govt.nz) for HDEC requirements relating to amendments and other post-approval processes.

\section{Your next progress report is due by 25 June 2020.}

The Northern B Health and Disability Ethics Committee is satisfied that your study is not a clinical trial that is to be conducted principally for the benefit of the manufacturer or distributor of the medicine or item being trialled. Participants injured as a result of treatment received as part of your study may therefore be eligible for publicly-funded compensation through the Accident Compensation Corporation (ACC).

Please don't hesitate to contact the HDEC secretariat for further information. We wish you all the best for your study.

Yours

sincerely,

Mrs Kate O'Connor Chairperson

Northern B Health and Disability Ethics Committee 